\title{
ALGORITHMS FOR CHOW-HEEGNER POINTS VIA ITERATED INTEGRALS
}

\author{
HENRI DARMON, MICHAEL DAUB, SAM LICHTENSTEIN, AND VICTOR ROTGER
}

With an appendix by William Stein

\begin{abstract}
Let $E_{/ \mathrm{Q}}$ be an elliptic curve of conductor $N$ and let $f$ be the weight 2 newform on $\Gamma_{0}(N)$ associated to it by modularity. Building on an idea of S. Zhang, an article by Darmon, Rotger, and Sols describes the construction of so-called Chow-Heegner points, $P_{T, f} \in E(\overline{\mathbf{Q}})$, indexed by algebraic correspondences $T \subset X_{0}(N) \times X_{0}(N)$. It also gives an analytic formula, depending only on the image of $T$ in cohomology under the complex cycle class map, for calculating $P_{T, f}$ numerically via Chen's theory of iterated integrals. The present work describes an algorithm based on this formula for computing the Chow-Heegner points to arbitrarily high complex accuracy, carries out the computation for all elliptic curves of rank 1 and conductor $N<100$ when the cycles $T$ arise from Hecke correspondences, and discusses several important variants of the basic construction.
\end{abstract}

\section{INTRODUCTION}

Let $E_{/ \mathbf{Q}}$ be an elliptic curve of conductor $N$ and let

$$
f=\sum_{n \geq 1} a_{n} e^{2 \pi i n z} \in S_{2}\left(\Gamma_{0}(N)\right)
$$

be the weight two newform associated to $E$ by modularity. The proof of the Tate conjecture for curves implies the existence of a nonconstant modular parametrisation

$$
\pi_{E}: X_{0}(N) \longrightarrow E
$$

defined over $\mathbf{Q}$, together with a regular differential $\omega \in \Omega^{1}(E / \mathbf{Q})$ satisfying

$$
\pi_{E}^{*}(\omega)=\omega_{f}:=2 \pi i f(z) d z .
$$

The map $\pi_{E}$ plays a key role in the study of the Birch and Swinnerton-Dyer conjecture (BSD). This conjecture predicts that, for any number field $H$, the rank of $E(H)$ is equal to the order of vanishing $r_{\text {an }}\left(E_{/ H}\right)$ of the $L$-series $L\left(E_{/ H}, s\right)$ at $s=1$; one is thus led to seek strategies for constructing a nontorsion point $P \in E(H)$ when $L\left(E_{/ H}, 1\right)=0$, or-better yet-a system of $r_{\text {an }}\left(E_{/ H}\right)$ independent such points.

When $H$ is a ring class field of an imaginary quadratic field $K$, a fruitful approach to this question is afforded by the theory of Heegner points attached to $K$. These are the images under $\pi_{E}$ of points in $X_{0}(N)(\bar{K})$ attached to the moduli of elliptic curves with complex multiplication by some order in $K$. For instance it is known, thanks to the work of Gross-Zagier GrZa and its strengthening by Zhang [Zh] combined with a mild generalisation BD1] of the work of Kolyvagin that Heegner points

Received by the editor December 23, 2011 and, in revised form, October 28, 2013 and December $23,2013$.

2010 Mathematics Subject Classification. Primary 11F67, 11G05, 11Y16, 14C15. 
attached to $K$ can be made to generate all $E(H) \otimes \mathbf{Q}$ when $r_{\text {an }}\left(E_{/ H}\right)=[H: K]$, at least when the discriminant of $K$ is prime to $N$ and $L\left(E_{/ K}, s\right)$ has a simple zero at $s=1$.

Let

$$
\mathfrak{H}^{*}=\{z \in \mathbf{C}: \operatorname{Im}(z)>0\} \cup \mathbf{P}^{1}(\mathbf{Q})=\mathfrak{H} \cup \mathbf{P}^{1}(\mathbf{Q})
$$

denote the extended upper half-plane, topologized in the usual manner. The Heegner points attached to $K$ correspond precisely to the quadratic irrationalities $z \in \mathfrak{H} \cap K$ under the usual identification of $X_{0}(N)(\mathbb{C})$ with $\Gamma_{0}(N) \backslash \mathcal{H}^{*}$. Furthermore, after identifying $E(\mathbb{C})$ with the quotient $\mathbb{C} / \Lambda_{E}$ by the period lattice $\Lambda_{E} \subset \mathbb{C}$ attached to the regular differential $\omega$ of (1.0.2), the map $\pi_{E}$ can be computed analytically from the formula

$$
\pi_{E}(z)=\int_{i \infty}^{z} \omega_{f}=\sum_{n=1}^{\infty} \frac{a_{n}}{n} e^{2 \pi i n z} \in \mathbb{C} / \Lambda_{E},
$$

where the integral is taken over the vertical line joining the cusp $i \infty \in \mathbf{P}^{1}(\mathbf{Q})$ to $z$ (or any path in $\Gamma_{0}(N) \backslash \mathcal{H}^{*}$ homotopic to it).

Equation (1.0.3) makes it possible to calculate Heegner points numerically on a computer 1 , and leads to an efficient algorithm for calculating algebraic points on elliptic curves which is presumably better than the more general-purpose method of descent, at least in situations where it can be made to yield nontorsion points. In addition, a careful study of (1.0.3) has guided attempts to unearth more general analytic recipes for algebraic points on elliptic curves in settings falling outside the scope of the theory of complex multiplication. To elaborate on this last point, let us mention that:

- In [BD2] and [Gr], the complex upper half-plane $\mathfrak{H}^{*}$ is replaced by its p-adic counterpart $\mathcal{H}_{p}:=\mathbb{P}_{1}\left(\mathbb{C}_{p}\right)-\mathbb{P}_{1}\left(\mathbf{Q}_{p}\right)$ regarded as a rigid analytic variety, and the group $\Gamma_{0}(N)$ by the group of units in a $\mathbb{Z}[1 / p]$-order of a totally definite quaternion algebra. Replacing the complex integrals of (1.0.3) by Coleman's $p$-adic path integrals leads to $p$-adic analytic formulae for (and explicit calculations of) Heegner points arising from Shimura curve parametrisations, thanks to the Cerednik-Drinfeld theory of $p$-adic uniformisation of these curves.

- By mimicking the formulae for Shimura curve parametrisations involving $p$-adic path integrals, the article [D1] was able to propose a $p$-adic construction of points on elliptic curves - the so-called Stark-Heegner points conjecturally defined over abelian extensions of real quadratic fields. In this setting, the global structure analogous to (1.0.1) is not currently known to be available, and numerical verifications such as those carried out in DP] provide the best evidence at present for the conjectures of [D1].

- Let $E_{/ F}$ be a modular elliptic curve over a totally real number field $F$. By calculations similar to those of [D1], but replacing (1.0.3) by a formula involving integrals of complex-analytic differential forms of higher order associated to Hilbert modular forms, [D2, Ch. VIII] proposes a purely complex construction of Stark-Heegner points in $E(\mathbf{C})$. These points are conjectured to be defined over suitable abelian extensions of quadratic extensions $K / F$ which have exactly one nonreal archimedean place. In this complex setting

\footnotetext{
${ }^{1}$ For the important role of experiments in the early study of Heegner points see, for example, Bi], BG].
} 
as well, numerical experiments such as those described in [DL] are the best evidence so far for the algebraicity of the Stark-Heegner points.

- Complex Stark-Heegner points can be viewed as images of certain nullhomologous topological cycles on Hilbert modular varieties under a kind of "Abel-Jacobi map". In the hope of better understanding this construction, the articles [BDP2] and [DRS] discuss "generalised modular parametrisations"

$$
\Pi: \mathrm{CH}^{r+1}(V)_{0} \longrightarrow E
$$

from the Chow group $\mathrm{CH}^{r+1}(V)_{0}$ of null-homologous codimension $r+1$ algebraic cycles on a $(2 r+1)$-dimensional variety $V$ to the Mordell-Weil group of an elliptic curve $E$, arising from suitable algebraic correspondences $\Pi \in \mathrm{CH}^{r+1}(V \times E)$. In the setting of BDP2], $E$ is an elliptic curve with complex multiplication, $V$ the product of an $(r+1)$-dimension Kuga-Sato variety with $E^{r}$, and the existence of $\Pi$ relies on an ostensibly difficult instance of the Hodge or Tate conjectures. Nonetheless, the global nature of the points in the image of $\Pi$, for which the term "Chow-Heegner point" was proposed in BDP2, is better understood than in the setting of StarkHeegner points.

An even simpler instance of the Chow-Heegner point construction arises when $V=$ $X_{0}(N)^{3}$ is the triple product of a modular curve and $\Pi \subset X_{0}(N)^{3} \times E$ is the product of a codimension 1 cycle $T \subset X_{0}(N)^{2}$ with the graph of the "classical" modular parametrisation $\pi_{E}$ of equation (1.0.1). The resulting map,

$$
\Pi_{T}: \mathrm{CH}^{2}\left(X_{0}(N)^{3}\right)_{0} \longrightarrow E,
$$

is a natural generalisation of (1.0.1). In particular, the image $P_{T, f}:=\Pi_{T}\left(\Delta_{\mathrm{GKS}}\right)$ of the Gross-Schoen modified diagonal cycle $\Delta_{\mathrm{GKS}}$ (as defined in Theorem 1 of [DRS] for example) under $\Pi_{T}$ is an interesting rational point on $E$ whose relations with special values of $L$-functions $[\mathrm{YZZ}$ and the pro-nilpotent fundamental groups of modular curves DRS] are well documented in the literature. The point $P_{T, f} \in E(\mathbf{Q})$ shall be called the Chow-Heegner point attached to the cycle $T$ and the newform $f$. An alternate, somewhat simpler description of $P_{T, f}$ proceeds by essentially intersecting $T$ with the diagonal in $X_{0}(N) \times X_{0}(N)$ to obtain a class in $\operatorname{Pic}\left(X_{0}(N)\right)$, and hence (after a suitable modification to make it of degree 0 ) a rational point $P_{T}$ of the Jacobian $J_{0}(N)$. The point $P_{T, f}$ is then just the image of $P_{T}$ under $\pi_{E}$. The definition of the points $P_{T}$ and $P_{T, f}$ is described more precisely in $₫ 2.3$ and $\$ 3.1$ below.

The article [DRS] also gives a complex analytic formula for the point $P_{T, f}$ in terms of K.-T. Chen's iterated path integrals. In order to provide some motivation for it, note that the homomorphism

$$
\pi_{1}\left(X_{0}(N)(\mathbf{C}) ; \infty\right) \longrightarrow \mathbf{C} \quad \text { defined by } \quad \gamma \mapsto \int_{\gamma} \omega_{f}
$$

factors through the maximal abelian quotient $H_{1}\left(X_{0}(N)(\mathbf{C}), \mathbf{Z}\right)$ of the fundamental group of $X_{0}(N)(\mathbb{C})$. Chen observed that it is also possible to obtain nonabelian information about this fundamental group through integration. His theory of iterated integrals gives rise to the functions

$$
J: \pi_{1}\left(X_{0}(N)(\mathbf{C}) ; \infty\right) \longrightarrow \mathbf{C}
$$


attached to an $r$-tuple of differentials, which does not need to factor through $H_{1}\left(X_{0}(N)(\mathbf{C}), \mathbf{Z}\right)$ but rather through the quotient of $\pi_{1}\left(X_{0}(N)(\mathbb{C}), \mathbb{Z}\right)$ by the $r$-th term in its lower central series. The algorithm for the computation of the ChowHeegner points $P_{T, f}$ described in this paper, in effect, replaces the integral appearing in (1.0.3) by a sum of iterated integrals attached to pairs of modular forms. These pairs depend only on the so-called Hodge cycle attached to $T$, namely, its image

$$
\xi_{T} \in H_{\mathrm{dR}}^{1,1}\left(X_{0}(N)^{2}\right) \cap H_{B}^{2}\left(X_{0}(N)^{2}, \mathbf{Q}\right)
$$

under the cycle class map. While algebraic equations for the modular curve $X_{0}(N)$ (and, a fortiori, for the cycle $T$ itself) can be hard to calculate in practice, the Hodge class $\xi_{T}$ is amenable to numerical calculation using the explicit description of the de Rham cohomology of modular curves in terms of weakly holomorphic modular forms of weight two and their $q$-expansions. The algorithm for computing the Hodge class $\xi_{T}$ and the associated iterated integrals has been implemented in a computer program using the free software package Sage $\left[S^{+} 09\right]$, and makes the Chow-Heegner points $P_{T, f}$ computable in practice for elliptic curves $E$ of moderate conductor.

As explained in the appendix, there are other, somewhat more direct approaches to computing $P_{T, f}$ for specific cycles $T$ arising from elliptic curve factors of $J_{0}(N)$. The approach based on iterated integrals appears to be somewhat more general and works for arbitrary Hodge cycles. In future work, we hope to extend our algorithm to compute Chow-Heegner points associated to "exceptional" Hodge cycles on products of Kuga-Sato varieties arising from CM forms. The rationality of ChowHeegner points computed in this manner would provide some indirect numerical evidence for certain open cases of the Hodge conjecture, in the spirit of the study carried out in BDP2].

We close this introduction by mentioning that there also exist $p$-adic methods for computing the points considered in this article, arising from [DR], where the authors provide explicit formulas for the image of the cycle $\Delta_{\text {GKS }}$ under the $p$ adic syntomic Abel-Jacobi map, involving special values of Garrett-Hida $p$-adic $L$-functions and " $p$-adic iterated integrals". The reader is referred to [Dau13, La], and the forthcoming [DLR] for more details on the $p$-adic approach.

Plan of the paper. In $\oint_{2}$ we recall necessary facts about iterated integrals and related ingredients for our main algorithm. In $\$ 3$ we specialize to the case of modular curves, define the points $P_{T, f}$ precisely, and write down an explicit analytic formula for them. In $\$ 4$ we describe in detail an algorithm for evaluating this formula numerically. The algorithm is illustrated with numerical examples in $\$ 5$, which also includes a table of triple Chow-Heegner points on rank one elliptic curves of small conductor.

Source code. Our Sage implementation of the algorithm described in this paper can be found at http://math. berkeley.edu/ mwdaub.

\section{Preliminaries}

2.1. De Rham cohomology. Let $X$ be a smooth, complete algebraic curve of genus $g \geq 2$ over $\mathbf{Q}$, and let $Y=X \backslash\{\infty\}$ be the complement of a single point in $X(\mathbf{Q})$. For a smooth variety $V$ defined over $\mathbf{Q}$ (such as $X$ or $Y$ ) we denote by $V^{\text {an }}$ the complex manifold $V(\mathbf{C})$ with its analytic topology. 
The de Rham cohomology $H_{\mathrm{dR}}^{1}\left(X^{\mathrm{an}}, \mathbf{C}\right)$ is the cohomology of the de Rham complex of smooth $\mathbf{C}$-valued differential forms on $X^{\text {an }}$.

Since the Riemann surface $X^{\text {an }}$ is algebraic and defined over $\mathbf{Q}$, there is a canonical isomorphism

$$
H_{\mathrm{dR}}^{1}\left(X^{\mathrm{an}}, \mathbf{C}\right)=H_{\mathrm{dR}}^{1}(X / \mathbf{Q}) \otimes \mathbf{C}
$$

Here,

$$
H_{\mathrm{dR}}^{1}(X / \mathbf{Q}):=\mathbf{H}^{1}\left(0 \rightarrow \mathcal{O}_{X} \rightarrow \Omega_{X}^{1} \rightarrow 0\right)
$$

is the algebraic de Rham cohomology of $X / \mathbf{Q}$, defined as the hypercohomology of the de Rham complex of sheaves of regular differential forms on $X$.

Because $X$ is a curve, the group $H_{\mathrm{dR}}^{1}(X / \mathbf{Q})$ has a particularly simple description in terms of the space $\Omega_{\mathrm{II}}^{1}(X)$ of differentials of the second kind on $X$, defined as

$\Omega_{\mathrm{II}}^{1}(X):=$ rational 1 -forms on $X$ with vanishing residues at all points of $X$.

Observe that $\Omega_{\mathrm{II}}^{1}(X)=\Omega_{\mathrm{II}}^{1}(Y)$, by the residue formula. Thus there is a canonical isomorphism

$$
H_{\mathrm{dR}}^{1}(X / \mathbf{Q})=\Omega_{\mathrm{II}}^{1}(Y) / \mathrm{d} \mathbf{Q}(Y),
$$

where $\mathbf{Q}(Y)=\mathbf{Q}(X)$ is the field of rational functions on $Y$ (or on $X$ ). Using the Riemann-Roch theorem, one sees that

$$
\Omega_{\mathrm{II}}^{1}(Y) / \mathrm{d} \mathbf{Q}(Y) \cong \Omega^{1}(Y) / \mathrm{d} \mathcal{O}(Y) .
$$

So $H_{\mathrm{dR}}^{1}(X / \mathbf{Q})$ can also be computed as the space of regular 1-forms on $Y$, modulo exact forms. For computational purposes, the latter description is the most useful: we will compute with classes in $H_{\mathrm{dR}}^{1}(Y)$ using rational 1-forms on $X$ which are regular away from the point $\infty$. These are amenable to computation via their Laurent expansions about $\infty$.

Later on we shall make use of the symplectic Poincaré pairing

$$
\langle,\rangle: H_{\mathrm{dR}}^{1}\left(X^{\mathrm{an}}, \mathbf{C}\right) \times H_{\mathrm{dR}}^{1}\left(X^{\mathrm{an}}, \mathbf{C}\right) \rightarrow \mathbf{C},
$$

defined by

$$
\langle\omega, \eta\rangle:=\frac{1}{2 \pi i} \int_{X} \omega \wedge \eta
$$

for (the cohomology classes of) smooth $\mathbf{C}$-valued 1-forms $\omega, \eta$ on $X^{\text {an }}$. If $\omega$ and $\eta$ are differentials of the second kind on $X$, regular away from the cusp $\infty$, then the induced pairing on $H_{\mathrm{dR}}^{1}(X / \mathbf{Q})$ can be computed as

$$
\langle\omega, \eta\rangle=\operatorname{res}_{\infty}\left(F_{\omega} \cdot \eta\right)=-\operatorname{res}_{\infty}\left(\omega \cdot F_{\eta}\right),
$$

where $F_{\nu}$ denotes a local primitive of the differential $\nu$ at $\infty$.

2.2. Iterated integrals. We now turn to recalling the definition and basic properties of iterated integrals; see [Ch], [H1], [H2 for more details.

Fix a base point $o \in Y^{\text {an }}$ and denote by $\Gamma:=\pi_{1}\left(Y^{\text {an }} ; o\right)$ the fundamental group of the Riemann surface $Y^{\text {an }}$. We write $I \subset \mathbf{Z}[\Gamma]$ for the augmentation ideal of the integral group ring of $\Gamma$. Recall that $H_{1}\left(X^{\mathrm{an}}, \mathbf{Z}\right)=H_{1}\left(Y^{\mathrm{an}}, \mathbf{Z}\right) \cong \Gamma^{\mathrm{ab}}$, as can be seen from the well-known presentation for the fundamental group of a Riemann surface, and that this abelian group is naturally identified with $I / I^{2}$.

Definition 2.2.1. The path space on $Y$ based at $o$, denoted $\mathbf{P}(Y ; o)$, is the set of piecewise-smooth paths

$$
\gamma:[0,1] \longrightarrow Y^{\text {an }}, \quad \text { with } \gamma(0)=o .
$$


Let

$$
\pi: \tilde{Y} \rightarrow Y^{\text {an }}, \quad \text { resp. } \quad \pi: \tilde{X} \rightarrow X^{\text {an }},
$$

denote the universal covering space of $Y^{\text {an }}$ (resp. $X^{\text {an }}$ ) corresponding to the basepoint $o$. The group $\Gamma$ acts on $\tilde{Y}$ transitively and without fixed points, and the map $\gamma \mapsto \gamma(1)$ identifies the quotient $\tilde{Y} / \Gamma$ with $Y^{\text {an }}$.

Recall that a closed, $\mathbf{C}$-valued smooth 1-form (resp. a meromorphic 1-form of the second kind) $\eta$ on $X^{\text {an }}$ admits a smooth (resp. meromorphic) primitive $F_{\eta}: \tilde{X} \rightarrow \mathbf{C}$, defined by the rule

$$
F_{\eta}(\gamma):=\int_{0}^{1} \gamma^{*} \eta
$$

Definition 2.2.2. The basic iterated integral attached to an ordered $n$-tuple $\left(\omega_{1}, \ldots, \omega_{n}\right)$ of smooth 1 -forms on $Y^{\text {an }}$ is the function $\mathbf{P}(Y ; o) \rightarrow \mathbf{C}$, denoted $\int \omega_{1} \cdot \omega_{2} \cdot \ldots \cdot \omega_{n}$, defined by

$$
\gamma \mapsto \int_{\gamma} \omega_{1} \cdot \omega_{2} \cdot \ldots \cdot \omega_{n}:=\int_{\Delta}\left(\gamma^{*} \omega_{1}\right)\left(t_{1}\right)\left(\gamma^{*} \omega_{2}\right)\left(t_{2}\right) \cdots\left(\gamma^{*} \omega_{n}\right)\left(t_{n}\right),
$$

where $\Delta$ is the simplex in $[0,1]^{n}$ defined by $0 \leq t_{n} \leq t_{n-1} \leq \cdots \leq t_{1} \leq 1$. The integer $n$ is called the length of this basic iterated integral.

Example 2.2.3. When $n=2$, the basic iterated integral attached to $\omega$ and $\eta$ can be computed by the formula

$$
\int_{\gamma} \omega \cdot \eta=\int_{\gamma} \omega F_{\eta}=\int_{0}^{1} \gamma^{*}\left(\omega F_{\eta}\right) .
$$

In the expression in the middle, we abusively use the same notation $\omega$ for the differential $\pi^{*} \omega$ on $\tilde{Y}$. The 1-form $\omega F_{\eta}$ is to be integrated along a lift of $\gamma$ to $\tilde{Y}$, which is unique once a lift of $o$ to $\tilde{Y}$ is specified.

Definition 2.2.4. An iterated integral is a linear combination of basic iterated integrals, viewed as a function on $\mathbf{P}(Y ; o)$. Its length is defined to be the maximum of the lengths of its constituent basic iterated integrals. It is said to be homotopy invariant if its value on any path $\gamma$ depends only on the homotopy class of $\gamma$.

A homotopy-invariant iterated integral defines a $\mathbf{C}$-valued function on $\Gamma$, and by extending linearly, induces a homomorphism of abelian groups $\mathbf{Z}[\Gamma] \rightarrow \mathbf{C}$. Observe that a homotopy invariant iterated integral of length $\leq n$ vanishes on the $(n+1)$ st power $I^{n+1}$ of the augmentation ideal in $\mathbf{Z}[\Gamma]$, and hence gives rise to a well-defined element of $\operatorname{Hom}\left(I / I^{n+1}, \mathbf{C}\right)$. The natural map

$\{$ homotopy invariant iterated integrals of length $\leq n\} \longrightarrow \operatorname{Hom}\left(I / I^{n+1}, \mathbf{C}\right)$

is an isomorphism; see, for example, [H2].

We will be interested in numerically evaluating certain homotopy invariant iterated integrals on $Y$ of length $\leq 2$. Suppose $\omega$ and $\eta$ are two differentials of the second kind on $X$, regular on $Y$, representing cohomology classes $\omega, \eta \in H_{\mathrm{dR}}^{1}(X / \mathbf{Q})$ in the manner of 92.1 . The basic iterated integral $\int \omega \cdot \eta$ of length 2 is not generally homotopy invariant. But when either $\omega$ or $\eta$ is holomorphic on $X$ - i.e., has no pole at $\infty$ - a suitable modification of $\int \omega \cdot \eta$ will be homotopy invariant, as we now explain. 
Recall that a differential on a Riemann surface is said to have a logarithmic pole at a point if its expansion in terms of a local parameter $q$ at this point is of the form $\sum_{n=0}^{\infty} a_{n} q^{n} \frac{d q}{q}$. When $\omega$ is holomorphic at $\infty$, we let $\alpha_{\omega, \eta}$ be a meromorphic 1 -form on $X$ that is regular on $Y$ and is such that the induced differential $\omega F_{\eta}-\alpha_{\omega, \eta}$ on $\tilde{X}$ has at worst a logarithmic pole at (any point lying over) $\infty$. This condition is well-posed because the principal part of $\omega F_{\eta}$ at $\tilde{x} \in \tilde{X}$ depends only on the image $x$ of $\tilde{x}$; see [DRS, $\S 2]$. The form $\alpha_{\omega, \eta}$ exists - and in fact can even be taken to be algebraic and defined over $\mathbf{Q}$ - by Riemann-Roch. If $\omega$ is not holomorphic at $\infty$ but $\eta$ is, then we define $\alpha_{\omega, \eta}:=-\alpha_{\eta, \omega}$.

Lemma 2.2.5. Let $\omega$ and $\eta$ be as above, and assume that either $\omega$ or $\eta$ is holomorphic at $\infty$. Then the iterated integral $J_{\omega, \eta}:=\int \omega \cdot \eta-\alpha_{\omega, \eta}$ is homotopy-invariant.

Proof. The homotopy invariance of $J_{\omega, \eta}$ follows from the fact that $J_{\omega, \eta}(\gamma)=$ $\int_{\gamma} \omega F_{\eta}-\alpha_{\omega, \eta}$, and the 1-form on $\tilde{X}$ in the integrand is holomorphic when restricted to $\tilde{Y}$.

Remark 2.2.6. Note that if $\omega$ and $\eta$ are both holomorphic at $\infty$, then we can take $\alpha_{\omega, \eta}=0$.

Now consider an integral Hodge class $\xi \in H^{1}\left(X^{\text {an }}, \mathbf{Z}\right) \otimes H^{1}\left(X^{\text {an }}, \mathbf{Z}\right)$, a cohomology class of type $(1,1)$ in $H^{2}\left(X^{\text {an }} \times X^{\text {an }}, \mathbf{Z}\right)$ that lies in the Künneth component $H^{1}\left(X^{\text {an }}, \mathbf{Z}\right) \otimes H^{1}\left(X^{\text {an }}, \mathbf{Z}\right)$. Since $\xi$ is type $(1,1)$, we can choose a basis $\left\{\omega_{i}\right\}$ such that when we write $\xi=\sum c_{i, j} \omega_{i} \otimes \omega_{j}$, then either $\omega_{i}$ or $\omega_{j}$ is holomorphic at $\infty$ whenever $c_{i, j} \neq 0$. By the previous lemma, the iterated integral $J_{\xi}=\sum c_{i j} J_{\omega_{i}, \omega_{j}}$ is homotopy invariant

Lemma 2.2.7. Suppose that $\xi$ is an integral Hodge class on $X \times X$ as above. Using (2.2.1), identify $J_{\xi}$ with a homomorphism of abelian groups $I / I^{3} \rightarrow \mathbf{C}$. Then the restriction of $J_{\xi}$ to $I^{2} / I^{3}$ is $\mathbf{Z}$-valued and agrees with $\xi$ viewed as an element of

$$
\begin{aligned}
H^{1}\left(X^{\mathrm{an}}, \mathbb{Z}\right) \otimes H^{1}\left(X^{\mathrm{an}}, \mathbb{Z}\right) & \cong\left(H_{1}\left(X^{\mathrm{an}}, \mathbb{Z}\right) \otimes H_{1}\left(X^{\mathrm{an}}, \mathbb{Z}\right)\right)^{\vee} \\
& =\left(I / I^{2} \otimes I / I^{2}\right)^{\vee}=\left(I^{2} / I^{3}\right)^{\vee} .
\end{aligned}
$$

(Here $A^{\vee}$ denotes $\operatorname{Hom}(A, \mathbb{Z})$, for any abelian group $A$.)

Proof. See the discussion at the beginning of $\S 2$ of [DRS], and loc. cit., Lemma $1.1(2)$.

By Lemma 2.2.7 the map $J_{\xi}$ induces a homomorphism

$$
J_{\xi}: H_{1}\left(X^{\text {an }}, \mathbf{Z}\right)=I / I^{2} \rightarrow \mathbf{C} / \mathbf{Z} .
$$

The following observation, which is extended in Theorem 2.3.1 below to the entire Jacobian of $X$, is key in our approach to calculating Chow-Heegner points. Fix any holomorphic 1-form $\rho \in H^{1,0}\left(X_{\mathbf{C}}\right) \subset H^{1}\left(X^{\text {an }}, \mathbf{C}\right)$ corresponding to an elliptic curve factor $E$ of the Jacobian of $X$, and denote by $\Lambda$ the period lattice

$$
\Lambda:=\left\{\int_{\gamma} \rho, \quad \gamma \in H_{1}\left(X^{\text {an }}, \mathbb{Z}\right)\right\} \subset \mathbf{C}
$$

attached to $\rho$. The class $\gamma_{\rho} \in H_{1}\left(X^{\text {an }}, \mathbf{C}\right)$ that is Poincaré dual to $\rho$ actually belongs to $H_{1}\left(X^{\text {an }}, \mathbf{Z}\right) \otimes \Lambda$. Consequently, $J_{\xi}\left(\gamma_{\rho}\right)$ can be viewed as a well-defined element of $\mathbf{C} / \Lambda$, and hence of $E(\mathbb{C})$. 
2.3. Chow-Heegner points. Let $X_{1}, X_{2}$ denote copies of $X$, and $X_{12}$ the diagonal copy of $X$ in $X_{1} \times X_{2}$. We fix a choice of basepoint $\infty \in X$ and use it to identify $X_{1}$ and $X_{2}$ with the curves $X_{1} \times\{\infty\}$ and $\{\infty\} \times X_{2}$ respectively in $X_{1} \times X_{2}$. To a codimension one algebraic cycle $Z \subset X \times X=X_{1} \times X_{2}$ (defined over $\mathbf{Q}$ ) we associate the divisor class

$$
D_{Z}=\left(Z \cap X_{12}\right)-\left(Z \cap X_{1}\right)-\left(Z \cap X_{2}\right) .
$$

Define a corresponding degree-zero divisor class

$$
P_{Z}=D_{Z}-\operatorname{deg}\left(D_{Z}\right) o \in \operatorname{Pic}^{0}(X) .
$$

(Recall that $o \in X(\mathbf{Q})$ is a fixed base point which is different from the previously chosen base point $\infty$.)

We now state the iterated integral formula from [DRS] for the image of $P_{Z}$ under the Abel-Jacobi map

$$
\operatorname{AJ}_{X}: \operatorname{Pic}^{0}(X) \rightarrow \Omega^{1}\left(X^{\text {an }}\right)^{\vee} / H_{1}\left(X^{\text {an }}, \mathbf{Z}\right) .
$$

Let $\epsilon_{o}$ be the projector on $\operatorname{Pic}(X \times X)$ defined by

$$
\epsilon_{o}(Z)=Z-i_{1 *} \pi_{1 *} Z-i_{2 *} \pi_{2 *} Z
$$

where $\pi_{1}, \pi_{2}: X \times X \rightrightarrows X$ are the projections and $i_{1}, i_{2}: X \rightrightarrows X \times X$ are the inclusions of "vertical and horizontal" copies of $X$ over the basepoint $o$.

Let

$$
\operatorname{cl}\left(\epsilon_{o}-\right): \operatorname{Pic}(X \times X) \rightarrow H^{1}\left(X^{\text {an }}, \mathbf{Z}\right) \otimes H^{1}\left(X^{\text {an }}, \mathbf{Z}\right)
$$

denote the composition of the cycle class map and the projector $\epsilon_{o}$. (The effect of $\epsilon_{0}$ is to annihilate the $H^{2} \otimes H^{0}$ and $H^{0} \otimes H^{2}$ factors in the Künneth decomposition of the second Betti cohomology group of $X \times X$.) Suppose $\operatorname{cl}\left(\epsilon_{o} Z\right)$ is represented by $\sum_{i, j} c_{i, j} \omega_{i} \otimes \omega_{j}$, where $\omega_{i}$ or $\omega_{j}$ is holomorphic at $\infty$ whenever $c_{i, j} \neq 0$.

Theorem 2.3.1 ([DRS, Corollary 2.6]). The image

$$
\operatorname{AJ}_{X}\left(P_{Z}\right) \in \Omega^{1}\left(X^{\mathrm{an}}\right)^{\vee} / H_{1}\left(X^{\mathrm{an}}, \mathbf{Z}\right)
$$

is represented by the linear functional that maps $\rho \in \Omega^{1}\left(X^{\text {an }}\right)$ to

$$
\sum_{i, j} c_{i, j} \int_{\gamma_{\rho}}\left(\omega_{i} \cdot \omega_{j}-\alpha_{\omega_{i}, \omega_{j}}\right)+\operatorname{deg}\left(D_{Z}\right) \int_{o}^{\infty} \rho \in \mathbf{C},
$$

where $\gamma_{\rho} \in H_{1}\left(X^{\mathrm{an}}, \mathbf{C}\right)$ is Poincaré dual to $\rho \in H^{1,0}\left(X^{\mathrm{an}}\right) \subset H_{\mathrm{dR}}^{1}\left(X^{\mathrm{an}}, \mathbf{C}\right)$.

\section{Chow-Heegner Points on modular Curves}

We now specialize the discussion of the preceding section to the case of classical modular curves $X$. We shall define certain rational points on an arbitrary elliptic curve $E_{/ \mathbf{Q}}$ called Chow-Heegner points, such that the corresponding points of $E(\mathbf{C}) \cong \mathbf{C} / \Lambda_{E}$ can be computed using iterated path integrals via Theorem 2.3.1. For more details on the theory of elliptic curves, an interested reader should consult [Sil]. For modular curves and modular forms, [DS] is a good introductory text. Another good resource is [Stn], which focuses more on the computational aspects, including modular symbols. 
3.1. Definitions. Let $N>1$ be an integer and $X=X_{0}(N)$ denote the canonical model over $\mathbf{Q}$ of the classical modular curve of level $N$; write $J_{0}(N)$ for the Jacobian of $X_{0}(N)$. With this choice of $X$ we place ourselves in the setup of $\S 2$, taking the ground field $F$ to be $\mathbf{Q}$ and the point $\infty \in X(\mathbf{Q})$ to be the usual cusp at infinity. Thus $Y:=X_{0}(N)-\{\infty\}$. (Note that $Y$ differs from the usual open modular curve $Y_{0}(N)$.) For the moment we do not specify the basepoint $o \in Y^{\text {an }}$ used for topological constructions.

We write $S_{2}\left(\Gamma_{0}(N)\right)$ for the space of cuspidal weight 2 modular forms for $\Gamma_{0}(N)$. This space is canonically isomorphic to the vector space $\Omega^{1}\left(X^{\text {an }}\right)$ of holomorphic 1 -forms on $X$, via the map which associates to a modular form $f: \mathfrak{H}^{*} \rightarrow \mathbf{C}$ the 1form $\omega_{f}=2 \pi i f(z) \mathrm{d} z$ on $X$. We recall that if $f$ has Fourier expansion $\sum_{n>1} a_{n} q^{n}$, then a Laurent expansion for $\omega_{f}$ near $\infty$ is given by $\sum_{n \geq 1} a_{n} q^{n} \frac{\mathrm{d} q}{q}$.

Let $E$ be an elliptic curve over $\mathbf{Q}$ of conductor $N_{E}$ dividing $N$. Its isogeny class corresponds to a newform $f \in S_{2}\left(\Gamma_{0}\left(N_{E}\right)\right)$ with rational Fourier coefficients, which gives rise to a modular parametrization $\pi_{E}: J_{0}\left(N_{E}\right) \rightarrow E$, a morphism of abelian varieties defined over $\mathbf{Q}$.

Up to replacing $E$ by an isogenous elliptic curve (which is harmless for our constructions), we can and will assume throughout that $\operatorname{ker} \pi_{E}$ is connected. In the literature this choice is often referred as the optimal elliptic curve. In this case, assuming the Manin constant $c=1$, the Néron lattice of $E$ coincides with the period lattice $\Lambda_{f}$ of the differential $\omega_{f}=2 \pi i f(z) \mathrm{d} z \in \Omega^{1}\left(X_{0}\left(N_{E}\right)^{\text {an }}\right)$ corresponding to $f$.

The map $\pi_{E}$ can be computed on complex points explicitly, using the Abel-Jacobi isomorphism

$$
\mathrm{AJ}_{X}: J_{0}\left(N_{E}\right)(\mathbf{C}) \cong \Omega^{1}\left(X_{0}\left(N_{E}\right)^{\text {an }}\right)^{\vee} / H_{1}\left(X_{0}\left(N_{E}\right)^{\text {an }}, \mathbf{Z}\right)
$$

the Weierstrass uniformization $W: \mathbf{C} / \Lambda_{f} \cong E(\mathbf{C})$, and the analytic parametrization

$$
\pi_{E}^{\text {an }}: \Omega^{1}\left(X_{0}\left(N_{E}\right)^{\text {an }}\right)^{\vee} / H_{1}\left(X_{0}\left(N_{E}\right)^{\text {an }}, \mathbf{Z}\right) \rightarrow \mathbf{C} / \Lambda_{f} .
$$

The map $\pi_{E}^{\text {an }}$ sends the coset of a functional on $\Omega^{1}\left(X_{0}\left(N_{E}\right)^{\text {an }}\right)$ to the evaluation of that functional at $\omega_{f}$. Thus for $P_{\mathbf{C}} \in J_{0}\left(N_{E}\right)(\mathbf{C})$ we have

$$
\pi_{E}\left(P_{\mathbf{C}}\right)=W\left(\pi_{E}^{\mathrm{an}}\left(\mathrm{AJ}_{X_{0}\left(N_{E}\right)}\left(P_{\mathbf{C}}\right)\right)\right)=W\left(\mathrm{AJ}_{X_{0}\left(N_{E}\right)}\left(P_{\mathbf{C}}\right)\left(\omega_{f}\right)\right) .
$$

For each divisor $d$ of $N / N_{E}$ there is a degeneracy map $\pi_{d}: X_{0}(N) \rightarrow X_{0}\left(N_{E}\right)$ that induces by the Albanese universal property a morphism of abelian varieties $\pi_{d}: J_{0}(N) \rightarrow J_{0}\left(N_{E}\right)$, which we denote with the same symbol. Put $\pi_{E}^{d}=\pi_{E} \cdot \pi_{d}$ : $J_{0}(N) \rightarrow E$ for the composition of $\pi_{d}$ with $\pi_{E}$. When $d=1$, the map $\pi_{1}$ is the projection arising from the natural inclusion $\Gamma_{0}(N) \subseteq \Gamma_{0}\left(N_{E}\right)$ and we shall simply write $\pi_{E}^{1}=\pi_{E}$; it will be clear from the context whether the source of this map is $J_{0}(N)$ or $J_{0}\left(N_{E}\right)$.

Let $\mathbf{T}=\mathbf{Q}\left[\ldots, T_{n}, \ldots\right]_{n \geq 1}$ be the Hecke algebra of level $N$, and let $\mathbf{T}_{0}$ denote the subalgebra generated by Hecke operators $T_{n}$ such that $(n, N)=1$. Then

$$
\mathbf{T}_{0} \simeq \prod_{h} K_{h} \subseteq \mathbf{T} \simeq \prod_{h} L_{h}
$$

where $h$ runs over Galois conjugacy classes of newforms of all levels $M$ dividing $N$,

$$
K_{h}=\mathbf{Q}\left(\left\{a_{n}(h)\right\}_{n \geq 1}\right)
$$

is the number field generated by the Hecke eigenvalues of $h$, and, if $N_{h} \mid N$ stands for the level of $h$, the ring $L_{h}$ is a commutative Artinian $K_{h}$-algebra of dimension 
$\left[L_{h}: K_{h}\right]=\sigma\left(N / N_{h}\right)$, the number of factors of $N / N_{h}$ (cf. [Ka, [RS, Ch. 14]). Let $\operatorname{End}(\operatorname{Jac}(X))$ denote the ring of endomorphisms of $\operatorname{Jac}(X)$ which are defined over Q. The endomorphism algebra $\operatorname{End}^{0}(\operatorname{Jac}(X)):=\mathbf{Q} \otimes \operatorname{End}(\operatorname{Jac}(X))$ contains the Hecke algebra $\mathbf{T}_{0}$ as its center and $\mathbf{T}$ as a maximal commutative subalgebra (and $\operatorname{End}^{0}(\operatorname{Jac}(X)) \simeq \mathbf{T} \simeq \mathbf{T}_{0}$ when $N$ is prime $)$. More precisely,

$$
\operatorname{End}^{0}(\operatorname{Jac}(X))=\left\langle\mathbf{T},\left\{\delta_{d}\right\}_{d \mid \sigma\left(N / N_{h}\right)}\right\rangle \simeq \prod_{h} M_{\sigma\left(N / N_{h}\right)}\left(K_{h}\right),
$$

is spanned by the Hecke operators in $\mathbf{T}$ and the degeneracy operators $\delta_{d}$ defined e.g. in $\mathrm{Ka}$, and is isomorphic to the above product of matrix algebras.

Definition 3.1.1. For a divisor $M$ of $N$ and a newform $g \in S_{2}\left(\Gamma_{0}(M)\right)$, denote by

$$
T_{g} \in \mathbf{T}_{0} \cong \prod_{h} K_{h}
$$

the idempotent with 1 in the $K_{g}$ component and 0 elsewhere.

We shall also view $T_{g}$ as an idempotent of $\mathbf{T}$ and of $\operatorname{End}^{0}(\operatorname{Jac}(X))$ by the natural inclusion, so that $\mathbf{T}[g]:=T_{g} \cdot \mathbf{T}=L_{g}$ and $\operatorname{End}^{0}(\operatorname{Jac}(X))[g]:=T_{g} \cdot \operatorname{End}^{0}(\operatorname{Jac}(X))=$ $M_{\sigma(N / M)}\left(K_{g}\right)$.

There are natural isomorphisms

$$
\begin{aligned}
& \operatorname{End}(\operatorname{Jac}(X)) \otimes \mathbf{Q} \\
& \quad \simeq \operatorname{Corr}(X):=\operatorname{Pic}(X \times X) \otimes \mathbf{Q} /\left(\pi_{1}^{*} \operatorname{Pic}(X) \otimes \mathbf{Q} \oplus \pi_{2}^{*} \operatorname{Pic}(X) \otimes \mathbf{Q}\right) .
\end{aligned}
$$

Under these identifications, an endomorphism $T \in \operatorname{End}^{0}(\operatorname{Jac}(X))$ is associated to a divisor class in $\operatorname{Pic}(X \times X) \otimes \mathbf{Q}$, which we also denote by the same symbol, and is well-defined only up to horizontal and vertical divisors.

In $\$ 2.3$ a point $P_{T} \in \operatorname{Pic}^{0}(X)=J_{0}(N)(\mathbf{Q})$ was attached to a divisor $T$ on $X \times X$; this point only depends on the class of $T$ in $\operatorname{Pic}(X \times X) \otimes \mathbf{Q} /\left(\pi_{1}^{*} \operatorname{Pic}(X) \otimes \mathbf{Q} \oplus\right.$ $\left.\pi_{2}^{*} \operatorname{Pic}(X) \otimes \mathbf{Q}\right)$. This construction gives rise to a map

$$
\begin{aligned}
\operatorname{Pic}(X \times X) \otimes \mathbf{Q} & \rightarrow J_{0}(N)(\mathbf{Q}) \otimes \mathbf{Q} \\
T & \mapsto P_{T} .
\end{aligned}
$$

Note that both these divisor classes and the projector $\epsilon_{o}$ depend on the basepoint $o$, so we must now specify a particular choice of $o$. In the following definition, we fix the choice of basepoint o to be the cusp $0 \in X$, which is distinct from $\infty$ because $N>1$.

Definition 3.1.2. For an operator $T \in \operatorname{End}^{0}(\operatorname{Jac}(X))[g]$ and a divisor $d$ of $N / N_{E}$, define the Chow-Heegner point

$$
P_{T, f^{(d)}}=\pi_{E}^{d}\left(P_{T}\right) \in E(\mathbf{Q}) \otimes \mathbf{Q} .
$$

When we take $d=1$ we shall just write $P_{T, f}$ for $P_{T, f(d)}$; in addition, the ChowHeegner point associated with $T=T_{g}$ and $d=1$ shall be denoted $P_{g, f}$.

For any positive integer $n$, define $T_{g, n}:=T_{g} \cdot T_{n} \in \mathbf{T}$ as the product of $T_{g}$ and $T_{n}$, and define

$$
P_{g, f, n}:=P_{T_{g, n}, f} .
$$

Note that $P_{g, f}=P_{g, f, 1}$ and that $P_{g, f, n}$ is a multiple of $P_{g, f}$ if the eigenvalue $a_{n}(g)$ of $T_{n}$ acting on the $g$-isotypic component is rational. Thus, the points $P_{g, f, n}$ for $n>1$ are only of interest when $g$ has irrational Fourier coefficients or is an 
oldform. For a general discussion of the notion of the Chow-Heegner point, of which the construction above is a special case; see the introduction of BDP2] and [DRS].

Remark 3.1.3. To avoid the tensor products with $\mathbf{Q}$ in the definition of the above Chow-Heegner points $P_{T, f^{(d)}}$, one must work with an actual algebraic cycle on $X \times X$, as opposed to $T$ which is merely a $\mathbf{Q}$-linear combination of such cycles. Define the "denominator" $d_{T}$ of $T \in \mathbf{T}$ to be the smallest positive integer such that $d_{T} T$ lies in the integral Hecke algebra $\mathbf{T}_{\mathbf{Z}}:=\mathbf{Z}\left[\ldots, T_{n}, \ldots\right]$. Then $d_{T} P_{T, f(d)}$ belongs to $E(\mathbf{Q})$; see also 4.7

Put $d_{g, n}:=d_{T_{g, n}}$. We shall regard $d_{g, n} P_{g, f, n}$ as well-defined only modulo the torsion subgroup $E(\mathbf{Q})^{\text {tor }}$. One reason for this is that to obtain a computable formula for $P_{g, f, n}$ one can take the basepoint $o$ to be any cusp other than $\infty$. There being no distinguished choice, one is left with an ambiguity valued in the image in $E(\mathbf{Q})$ of the (torsion) cuspidal subgroup of $J_{0}(N)$; see (3.2.2) below and the subsequent remarks.

Theorem 3.1.4 ([DRS, Theorem 3.7]). Assume that the local signs $\varepsilon_{p}(g, g, f)$ of Garrett's triple product $L$-function $L(g, g, f, s)$ are +1 at the primes $p \mid N$. Then the module of points

$$
\underline{P}_{g, f}:=\left\langle P_{T, f^{(d)}}: T \in \operatorname{End}^{0}(\operatorname{Jac}(X))[g], d \mid \frac{N}{N_{E}}\right\rangle \subseteq E(\mathbf{Q}) \otimes \mathbf{Q}
$$

is nonzero (equivalently, there is a point $\sum_{d \mid N / N_{E}} n_{d} \pi_{E}^{d}\left(d_{T} P_{T}\right) \in E(\mathbf{Q})$ which is nontorsion, for some $T$ and integers $n_{d}$ ) if and only if the following conditions hold:

i. $L(f, 1)=0$,

ii. $L^{\prime}(f, 1) \neq 0$, and

iii. $L\left(f \otimes \operatorname{Sym}^{2}\left({ }^{\sigma} g\right), 2\right) \neq 0$ for all $\sigma \in \operatorname{Gal}(\overline{\mathbf{Q}} / \mathbf{Q})$.

3.2. Algorithms. The aim of this section is to explain how to explicitly compute the slightly smaller submodule

$$
\underline{P}_{\mathbf{T}[g], f}:=\left\{P_{T, f}: T \in \mathbf{T}[g]\right\}=\left\langle P_{g, f, n}: n \geq 1\right\rangle \subseteq \underline{P}_{g, f}
$$

of the Mordell-Weil group $E(\mathbf{Q}) \otimes \mathbf{Q}$ of $E$. Although the methods generalize to the computation of the full module of points $\underline{P}_{g, f}$, the computation of $\underline{P}_{\mathbf{T}[g], f}$ is simpler, and often sufficient for the purpose of finding a nontorsion point when one exists by Theorem 3.1.4. Indeed, the authors have not come across an example where $\underline{P}_{\mathbf{T}[g], f}$ is torsion but the a priori larger $\underline{P}_{g, f}$ is nontorsion. The interested reader may consult $\mathrm{Ka}$. for details relevant to the eventual adaptations of our methods to calculating $\underline{P}_{g, f}$ in its entirety.

Theorem 2.3.1 gives rise to an explicit formula for a Chow-Heegner point $P_{g, f, n}$ in terms of iterated integrals. This formula is stated in terms of the components of the cohomology class $\operatorname{cl}\left(\epsilon_{o} T_{g, n}\right) \in H_{\mathrm{dR}}^{1}(X / \mathbf{Q})^{\otimes 2}$ expressed as a sum of pure tensors. In this section we find an explicit formula for $\operatorname{cl}\left(\epsilon_{o} T_{g, n}\right)$.

The action of the Hecke algebra $\mathbf{T}_{0}$ on modular forms extends to an action on the de Rham cohomology of $X$. Under this action, we have

$$
H_{\mathrm{dR}}^{1}(X / \mathbf{Q}) \cong H_{\mathrm{dR}}^{1}(X / \mathbf{Q})\left[g_{1}\right] \oplus \cdots \oplus H_{\mathrm{dR}}^{1}(X / \mathbf{Q})\left[g_{n}\right],
$$

indexed by Galois conjugacy classes of newforms of all levels $M$ dividing $N$. Let $g \in S_{2}\left(\Gamma_{0}(M)\right)$ be a newform and suppose $\left\{\omega_{g, 1}, \ldots, \omega_{g, 2 k}\right\}$ is a collection of differentials of the second kind on $X$ representing a basis for $H_{\mathrm{dR}}^{1}(X / \mathbf{Q})[g]$. Write 
$T_{n} \omega_{g, i}=\sum_{j} a_{i j}^{n} \omega_{g, j}$, and denote by $A_{n}$ and $B$ the matrices $\left(a_{i j}^{n}\right)_{1 \leq i, j \leq 2 k}$ and $\left(\left\langle\omega_{g, i}, \omega_{g, j}\right\rangle\right)_{1 \leq i, j \leq 2 k}$, respectively, where $\langle$,$\rangle denotes the Poincaré pairing. Then$ we have the following:

Lemma 3.2.1. $\operatorname{cl}\left(\epsilon_{o} T_{g, n}\right)=\sum_{i, j} c_{i j}^{n} \omega_{g, i} \otimes \omega_{g, j}$, where $\left(c_{i j}^{n}\right)_{1 \leq i, j \leq 2 k}=-B^{-1} A_{n}$.

Proof. The projector $\epsilon_{o}$ acts on $H_{\mathrm{dR}}^{2}(X \times X)$ by annihilating the $H_{\mathrm{dR}}^{0}(X) \otimes H_{\mathrm{dR}}^{2}(X)$ and $H_{\mathrm{dR}}^{2}(X) \otimes H_{\mathrm{dR}}^{0}(X)$ components of the Künneth decomposition, so we have

$$
\operatorname{cl}\left(\epsilon_{o} T_{g, n}\right) \in H_{\mathrm{dR}}^{1}(X) \otimes H_{\mathrm{dR}}^{1}(X) .
$$

Note from the definition that $T_{g, n}$ acts on $H_{\mathrm{dR}}^{1}(X)[h]$ as $T_{n}$ if $h=g$ and 0 otherwise, so $\operatorname{cl}\left(\epsilon_{o} T_{g, n}\right)$ is equal to the image of $T_{n}$ under the identification:

$$
\operatorname{End}\left(H_{\mathrm{dR}}^{1}(X)[g]\right) \simeq H_{\mathrm{dR}}^{1}(X)[g]^{\vee} \otimes H_{\mathrm{dR}}^{1}(X)[g] \simeq H_{\mathrm{dR}}^{1}(X)[g] \otimes H_{\mathrm{dR}}^{1}(X)[g] .
$$

The first map is the canonical isomorphism of finite dimensional vector spaces, and the second is induced from the inverse of the identification $H_{\mathrm{dR}}^{1}(X)[g] \simeq$ $H_{\mathrm{dR}}^{1}(X)[g]^{\vee}$ via the map $v \mapsto(w \mapsto\langle v, w\rangle)$. The remainder of the proof is a straightforward exercise in linear algebra, and is left to the reader.

Combining the previous results, we obtain the following formula for $P_{g, f, n}$. Let $\gamma_{f}$ be the Poincaré dual of $\omega_{f}$ and let $\omega_{g, 1}, \ldots, \omega_{g, 2 k}$ be differentials of the second kind that give rise to a symplectic basis for the $g$-isotypic $\mathbf{Q}$-subspace $H_{\mathrm{dR}}^{1}(X / \mathbf{Q})[g]$. That is, for $1 \leq i \leq k$ we have $\left\langle\omega_{g, i}, \omega_{g, i+k}\right\rangle=1$ and $\left\langle\omega_{g, i}, \omega_{g, j}\right\rangle=0$ for $j \neq$ $i+k$. Assume, moreover, that this basis is adapted to the Hodge filtration, in the sense that the 1 -forms $\omega_{g, 1}, \ldots, \omega_{g, k}$ are regular at $\infty$. Since $H^{0}\left(X, \Omega^{1}(X / \mathbf{Q})\right)[g] \subset$ $H_{\mathrm{dR}}^{1}(X / \mathbf{Q})[g]$ is a maximal isotropic subspace with respect to the Poincaré pairing, we can simply take any basis $\omega_{g, 1}, \ldots, \omega_{g, k}$ for $H^{0}\left(X, \Omega^{1}(X / \mathbf{Q})\right)[g]=S_{2}\left(\Gamma_{0}(N)\right)[g]$ and extend it to a symplectic basis of the sort desired. Write

$$
\operatorname{cl}\left(\epsilon_{o} T_{g, n}\right)=\sum_{i, j}^{2 k} c_{i j}^{n} \omega_{g, i} \otimes \omega_{g, j}
$$

where the coefficients can be computed as in Lemma 3.2.1. Since we chose the basis to be symplectic, the matrix $B$ is the standard symplectic matrix. Additionally, because $T_{n}$ preserves the holomorphic subspace of $H_{\mathrm{dR}}^{1}(X / \mathbf{Q})$, then the computation of $-B^{-1} A_{n}$ shows that $c_{i j}^{n}=0$ when $k+1 \leq i, j \leq 2 k$. Thus, the Hodge class in (3.2.1) is of the form discussed after Remark 2.2.6.

Combining (3.2.1) with (3.1.1) and Theorem 2.3.1, we obtain the following formula for the point $P_{g, f, n}$ :

$$
P_{g, f, n}=W\left(\sum_{i, j}^{2 k} c_{i j}^{n}\left(\int_{\gamma_{f}} \omega_{g, i} \cdot \omega_{g, j}-\alpha_{\omega_{g, i}, \omega_{g, j}}\right)\right) .
$$

Note that (3.2.2) omits the term $\operatorname{deg}\left(D_{T_{g, n}}\right) \int_{o}^{\infty} \omega_{f}$ from the formula in Theorem 2.3.1. This is justified by our choice of basepoint $o$, for it is well-known (cf. e.g. [Man]) that the difference $[\infty]-[0]$ is a torsion point of $J_{0}(N)(\mathbf{Q})$. Since $P_{g, f, n}$ is defined as an element of $E(\mathbf{Q}) \otimes \mathbf{Q}$, torsion can be disregarded.

We emphasize that by Lemmas 2.2.5 and 2.2.7, the right-hand side of (3.2.2) depends only on the homology class

$$
\gamma_{f} \in H_{1}\left(Y^{\mathrm{an}}, \mathbf{Z}\right)=H_{1}\left(X^{\mathrm{an}}, \mathbf{Z}\right)
$$


Poincaré dual to $\omega_{f}$. It can therefore be evaluated by lifting $\gamma_{f}$ arbitrarily to an element $\tilde{\gamma}_{f} \in \pi_{1}\left(Y^{\text {an }} ; o\right)$ and evaluating the length $\leq 2$ homotopy invariant iterated integral (3.2.2) on any loop in the homotopy class $\tilde{\gamma}_{f}$.

Remark 3.2.2. When $n=1$, formula (3.2.2) greatly simplifies. In the notation of Lemma 3.2.1, $A_{1}$ is the identity matrix and $B$ is the standard symplectic matrix by our choice of basis, and so if we write $\eta_{g, i}$ for $\omega_{g, i+k}$, then

$$
P_{g, f}=W\left(\sum_{i=1}^{k}\left(\int_{\gamma_{f}} \omega_{g, i} \cdot \eta_{g, i}-\eta_{g, i} \cdot \omega_{g, i}-2 \alpha_{\omega_{g, i}, \eta_{g, i}}\right)\right) .
$$

Although $P_{g, f, n}$ is defined relative to the choice of basepoint $o$ as the cusp 0 on $Y$, it will be convenient to compute it relative to a different choice of basepoint.

Lemma 3.2.3. The right-hand side of (3.2.2) is independent of the choice of $o \in$ $Y(\mathbf{Q})$.

Proof. Changing the basepoint from $o$ to $o^{\prime}$ amounts to conjugating the representative path $\gamma_{f}$ for the homology class Poincaré dual to $\omega_{f}$ by a path $\beta$ from $o$ to $o^{\prime}$. This manifestly does not affect the value of the integral of the meromorphic 1 -form $\alpha_{\omega_{g, i}, \omega_{g, j}}$. Thus the issue is whether we have an identity

$$
\int_{\gamma_{f}} \omega_{g, i} \cdot \omega_{g, j} \stackrel{?}{=} \int_{\beta \gamma_{f} \beta^{-1}} \omega_{g, i} \cdot \omega_{g, j}
$$

But by [H1, Exer. 8], for any 1-forms $\omega, \eta$, loop $\gamma$, and path $\beta$, we have

$$
\int_{\beta \gamma \beta^{-1}} \omega \cdot \eta=\int_{\gamma} \omega \cdot \eta+\left|\begin{array}{ll}
\int_{\gamma} \omega \int_{\gamma} \eta \\
\int_{\beta} \omega \int_{\beta} \eta
\end{array}\right|
$$

In our situation, the determinants expressing the difference between the two sides of (3.2.3) vanish. Indeed, $\int_{\gamma_{f}} \omega_{g, i}=\left\langle\omega_{f}, \omega_{g, i}\right\rangle=0$, since the decomposition into isotypic components for the action of the Hecke algebra is orthogonal with respect to the Poincaré pairing.

\section{Details of the Algorithm}

We now turn to the question of numerically evaluating formula (3.2.2) for a Chow-Heegner point $P_{g, f, n} \in E(\mathbf{Q}) \otimes \mathbf{Q}$ for an elliptic curve $E=E_{f}$. We retain all the notation from $\S ₫ 2$ and 3 , The following ingredients occur in (3.2.2):

1. The Poincaré dual $\gamma_{f} \in H_{1}(X, \mathbf{C})$ of $\omega_{f} \in H_{\mathrm{dR}}^{1}\left(X^{\mathrm{an}}, \mathbf{C}\right)$.

2. A collection of rational differentials of the second kind $\omega_{g, 1}, \ldots, \omega_{g, 2 k}$ on $X$, regular away from $\infty$, whose images in $H_{\mathrm{dR}}^{1}(X / \mathbf{Q})$ are a symplectic basis for the $g$-isotypic component $H_{\mathrm{dR}}^{1}(X / \mathbf{Q})[g]$. This basis should be adapated to the Hodge filtration on $H_{\mathrm{dR}}^{1}(X / \mathbf{Q})[g]$ in the sense that $\omega_{g, 1}, \ldots, \omega_{g, k}$ are holomorphic everywhere on $X$, including the point $\infty$.

3. The coefficients $c_{i j}^{n}$ arising from the action of $T_{n}$ on $H_{\mathrm{dR}}^{1}(X)$ with respect to the basis $\omega_{g, 1}, \ldots, \omega_{g, 2 k}$.

4. Meromorphic differentials $\alpha_{\omega_{g, i}, \omega_{g, j}}$ on $X$, regular on $Y$, such that $\omega_{g, i} F_{\eta_{g, j}}-$ $\alpha_{\omega_{g, i}, \eta_{g, j}}$ has at worst a logarithmic pole at (any point lying over) $\infty$ for $1 \leq i \leq k$.

These data must be "known" in a sufficiently concrete form to evaluate the iterated integrals occurring in (3.2.2). It is also desirable to know: 
5. The denominator $d_{g, n}$ of the projector onto the $g$-isotypic component of the cohomology of $X$.

This last item will allow for the computation of a point in $E(\mathbf{Q})$, as opposed to one in $E(\mathbf{Q}) \otimes \mathbf{Q}$. This section is devoted to methods of computing these five ingredients.

4.1. Evaluating iterated integrals. Let $J=\int \omega \cdot \eta-\alpha_{\omega, \eta}$ be a homotopyinvariant iterated integral of length $\leq 2$ on $Y$, expressed in terms of differentials of the second kind on $X$, regular on $Y$. We seek to compute the right-hand side of formula (3.2.2), which is a Q-linear combination of $J(\gamma)$ for various choices of $J$ and (the homotopy class of) a path $\gamma \in \pi_{1}(Y ; o)$. As remarked earlier, 3.2.2) actually depends only on the homology class $\gamma_{0}$ of $\gamma$. This homology class belongs to $H_{1}\left(Y^{\text {an }}, \mathbf{Z}\right)=H_{1}\left(X^{\text {an }}, \mathbf{Z}\right)$, which is the abelianization of the quotient $\pi_{1}\left(X^{\mathrm{an}}, o\right)=\bar{\Gamma}_{0}(N)$ of $\Gamma_{0}(N)$ by the smallest normal subgroup containing the elliptic and parabolic elements.

To evaluate $J\left(\gamma_{0}\right)$ for $\gamma_{0} \in H_{1}\left(Y^{\text {an }}, \mathbf{Z}\right)$, choose the basepoint $o$ away from the set $S$ of elliptic points on $Y_{0}(N)^{\text {an }} \subset Y^{\text {an }}$ and lift $\gamma_{0}$ arbitrarily to a path $\tilde{\gamma} \in \pi_{1}\left(Y_{0}(N)^{\text {an }} \backslash S, o\right)$. For each elliptic point $x \in S$, let $e_{x}=\left|\operatorname{Stab}_{\Gamma_{0}(N)}(x) /\{ \pm 1\}\right|$ denote the index of $x$ (which is either 2 or 3 ) and let $\gamma_{x}$ be a sufficiently small counterclockwise loop around $x$. Writing $H$ for the normal subgroup of $\pi_{1}\left(Y_{0}(N)^{\text {an }} \backslash S, o\right)$ generated by $\left\{\gamma_{x}^{e_{x}}, x \in S\right\}$, there is a natural isomorphism $\Gamma_{0}(N) \simeq \pi_{1}\left(Y_{0}(N)^{\text {an }}-\right.$ $S ; o) / H$.

We may regard then $\tilde{\gamma}$ as an element of $\Gamma_{0}(N)$; this causes no ambiguity because $H$ lies in the kernel of the natural projection $H_{1}\left(Y_{0}(N)^{\text {an }}-S, \mathbf{Z}\right)$ $\rightarrow H_{1}\left(Y_{0}(N)^{\text {an }}, \mathbf{Z}\right) \rightarrow H_{1}\left(Y^{\text {an }}, \mathbf{Z}\right)$. The path $\tilde{\gamma}$ can then also be viewed as a path in $\mathfrak{H}$ from $\tau_{0}$ to $\gamma \tau_{0}$, where $\gamma \in \Gamma_{0}(N)$ is a lift of $\gamma_{0}$.

Lemma 4.1.1. Suppose $\gamma_{0}$ is Poincaré dual to $\rho$. As an element of $\mathbf{C} / \Lambda_{\rho}$, we have

$$
J\left(\gamma_{0}\right)=\int_{\tau_{0}}^{\gamma \tau_{0}} \omega F_{\eta}-\alpha_{\omega, \eta}
$$

where we conflate 1-forms on $X$ with their pullbacks to $\mathfrak{H}^{*}=\mathfrak{H} \cup\{\infty\}$. Moreover, $F_{\eta}$ has Laurent expansion about $\infty \in \mathfrak{h}^{*}$ given by formally integrating the Laurent expansion of $\eta$ about the cusp $\infty \in X$.

Proof. This follows from the preceding discussion, using the definition of iterated integrals and the homotopy invariance of $J$.

Given any differential form $\lambda$ of the second kind on $X$, and any $\gamma \in \Gamma_{0}(N)$, let

$$
I(\lambda ; \gamma):=\int_{\tau_{0}}^{\gamma \tau_{0}} \lambda
$$

(As above, in the right-hand side of this expression $\lambda$ is conflated with its pullback to $\mathfrak{H}^{*}$.) By the residue formula, this expression is independent of the choice of path on the upper half-plane $\mathfrak{H}$ from $\tau_{0}$ to $\gamma \tau_{0}$. The $\Gamma_{0}(N)$-invariance of $\lambda$ also shows that this expression is independent of the choice of basepoint $\tau_{0} \in \mathfrak{H}$, which justifies suppressing $\tau_{0}$ from the notation.

If $\lambda$ instead denotes a differential of the second kind on $\tilde{X}$ then the integral above still makes sense but depends on both the basepoint $o$ and the chosen lift of $o$ to $\tau_{0} \in \mathfrak{H}$. We will primarily be interested in evaluating such integrals in the context of (3.2.2), for which the choice of basepoint is ultimately irrelevant. 
(This is because the Poincare dual of the homology class of $\gamma$ is orthogonal to the 1-forms in the iterated integral giving rise to the path integral we seek to evaluate; cf. Lemma 3.2.3.) However, as we are about to see, for the purposes of algorithmic efficiency it is necessary to break up the path of integration into pieces that can be computed relatively quickly. The integrals over these pieces may no longer be basepoint-independent: when we express $\gamma$ as a product of computationallyamenable elements $\gamma^{(j)} \in \Gamma_{0}(N)$, the corresponding homology classes may no longer lie in (the Poincaré dual of) the orthogonal complement of $H_{\mathrm{dR}}^{1}(X / \mathbf{Q})[g]$. Thus for a general meromorphic 1-form $\lambda$ on $\tilde{X}$ and a general $\gamma \in \Gamma_{0}(N)$, we adopt the notation

$$
I_{\tau_{0}}(\lambda ; \gamma)=\int_{\tau_{0}}^{\gamma \tau_{0}} \lambda
$$

to emphasize the dependence on the choice of basepoint.

By meromorphicity, for $\lambda$ as above (defined on either $X$ or $\tilde{X})$, the integral $I(\lambda ; \gamma)$ or $I_{\tau_{0}}(\lambda ; \gamma)$ can be computed by integrating termwise a Laurent expansion for $\lambda$ using the fundamental theorem of calculus. Thus, in practice, one computes the Laurent expansion for the primitive $F_{\lambda}$ about $\infty \in X$ (or a choice of $\tilde{\infty} \in \tilde{X}$ lying over $\infty$ ), regarded as a function given by a convergent power series in $q=e^{2 \pi i \tau}$ on $\mathfrak{h}$, and evaluates it at $\tau_{0}$ and $\tau_{0}^{\prime}=\gamma \tau_{0}$. The larger the imaginary parts of $\tau_{0}$ and $\tau_{0}^{\prime}$ are, the faster this series converges and the fewer coefficients of the Laurent series of $\lambda$ are necessary to approximate $I(\lambda ; \gamma)$ or $I_{\tau_{0}}(\lambda ; \gamma)$ to a given degree of accuracy. Writing $\gamma=\left(\begin{array}{ll}a & b \\ c & d\end{array}\right)$, it is well-known that the best compromise between $\operatorname{Im}\left(\tau_{0}\right)$ and $\operatorname{Im}\left(\tau_{0}^{\prime}\right)$ is achieved when we choose $\tau_{0}=-\frac{d}{c}+\frac{1}{|c|} i$ (cf., for example, [Cr, p. 35]). This optimal basepoint for $\gamma$ will be denoted $\tau_{\gamma}^{*}$.

With this remark in mind, we take the following approach to computing $J\left(\gamma_{0}\right)$ as in the lemma above. First compute Laurent expansions for the differentials $\omega, \eta, \alpha_{\omega, \eta}$. Then find a "good" expression for the homology class $\gamma_{0} \in H_{1}\left(X^{\text {an }}, \mathbf{C}\right)$, writing it as a $\mathbf{C}$-linear combination of classes $\gamma_{0}^{(j)} \in H_{1}\left(X^{\text {an }}, \mathbf{Z}\right)$ that lift to elements $\gamma^{(j)} \in \Gamma_{0}(N)$ with small lower-left entries $c N$. Finally, calculate approximations to the integrals $I_{\tau_{0}}\left(\omega F_{\eta} ; \gamma^{(j)}\right)$ and $I\left(\alpha_{\omega, \eta} ; \gamma^{(j)}\right)$. The appropriate linear combination of these integrals is an (approximate) representative for the coset $\left.J\left(\gamma_{0}\right) \in \mathbf{C} / \Lambda_{\rho}.\right)$

To calculate $I\left(\alpha_{\omega, \eta} ; \gamma^{(j)}\right)$, one is free to change the basepoint from $\tau_{0}$ to the optimal basepoint $\tau_{j}^{*}:=\tau_{\gamma^{(j)}}^{*}$ for $\gamma^{(j)}$, since $\alpha_{\omega, \eta}$ is defined on $X$ and not only on $\tilde{X}$. The same is not true for $\omega F_{\eta}$. To evaluate $I_{\tau_{0}}\left(\omega F_{\eta} ; \gamma^{(j)}\right)$ we appeal to the following lemma.

Lemma 4.1.2. $I_{\tau_{0}}\left(\omega F_{\eta} ; \gamma^{(j)}\right)=I_{\tau_{j}^{*}}\left(\omega F_{\eta} ; \gamma^{(j)}\right)-I\left(\eta ; \gamma^{(j)}\right) \int_{\tau_{0}}^{\tau_{j}^{*}} \omega$.

Observe that every term on the right-hand side can be computed using the fundamental theorem of calculus, evaluating power series only at the points $\tau_{0}$ and $\tau_{j}^{*}$. In particular, taking $\tau_{0}=i / N$, each such evaluation converges at least as fast as an evaluation at $\tau_{j}^{*}$, so this formula for the integral is "optimally efficient".

Proof of Lemma 4.1.2, Since $\lambda=\omega F_{\eta}$ is a holomorphic 1-form on $\mathfrak{H}$, its integral along a closed contour vanishes. Thus

$$
I_{\tau_{0}}\left(\lambda ; \gamma^{(j)}\right)=I_{\tau_{j}^{*}}\left(\lambda ; \gamma^{(j)}\right)+\int_{\tau_{0}}^{\tau_{j}^{*}} \lambda-\int_{\gamma^{(j)} \tau_{0}}^{\gamma^{(j)} \tau_{j}^{*}} \lambda .
$$


To evaluate the second term on the right-hand side, we observe that $\omega$ comes from a 1-form on $X$, so it is $\Gamma_{0}(N)$-invariant; it thus pulls back to itself along the fractional linear transformation defined by $\gamma^{(j)}$. On the other hand,

$$
I\left(\eta ; \gamma^{(j)}\right)=\int_{\gamma^{(j)}} \eta=F_{\eta}\left(\gamma^{(j)} \tau\right)-F_{\eta}(\tau), \quad \text { for all } \tau \in \mathfrak{H} .
$$

Hence $\left(\gamma^{(j)}\right)^{*} F_{\eta}=F_{\eta}+I\left(\eta ; \gamma^{(j)}\right)$. So $\left(\gamma^{(j)}\right)^{*} \lambda=\lambda+I\left(\eta ; \gamma^{(j)}\right) \omega$, and we find

$$
\int_{\gamma^{(j)} \tau_{0}}^{\gamma^{(j)} \tau_{j}^{*}} \lambda=\int_{\tau_{0}}^{\tau_{j}^{*}}\left(\gamma^{(j)}\right)^{*} \lambda=\int_{\tau_{0}}^{\tau_{j}^{*}} \lambda+I\left(\eta ; \gamma^{(j)}\right) \int_{\tau_{0}}^{\tau_{j}^{*}} \omega,
$$

which yields the lemma.

Remark 4.1.3. We warn the reader that possibly $I_{\tau_{0}}\left(\omega F_{\eta} ; \gamma^{(j)}\right) \neq \int_{\gamma^{(j)}} \omega \cdot \eta$ (regarding $\gamma^{(j)}$ as an element of $\left.\pi_{1}\left(Y^{\text {an }} ; o\right)\right)$. Indeed, the iterated integral attached to $\omega \cdot \eta$ need not even be homotopy invariant (!) so $\int_{\gamma(j)} \omega \cdot \eta$ is ill-defined. In particular, one cannot relate $I_{\tau_{0}}\left(\omega F_{\eta} ; \gamma^{(j)}\right)$ to $I_{\tau_{j}^{*}}\left(\omega F_{\eta} ; \gamma^{(j)}\right)$ using the change-of-basepoint formula (3.2.4) for iterated integrals.

To efficiently evaluate the integrals in (3.2.2) using the method just explained, it is therefore necessary to know:

a. Laurent expansions about $\infty$ for a symplectic basis $\omega_{g, 1}, \ldots, \omega_{g, 2 k}$ of $H_{\mathrm{dR}}^{1}(X / \mathbf{Q})[g]$ and the forms $\alpha_{\omega_{g, i}, \omega_{g, j}} ;$

b. the homology class $\gamma_{f}$ as a $\mathbf{C}$-linear combinationation of class $\gamma_{0}^{(j)}$ whose lifts to $\Gamma_{0}(N)$ have small lower-left entries $c N$.

In the rest of this section we turn to the task of computing these data.

4.2. Calculating a symplectic basis for $H_{\mathrm{dR}}^{1}(X / \mathbf{Q})[g]$. The calculation of a basis for the de Rham cohomology can be carried out by first writing down a modular function $u$ - that is, a rational function on $X=X_{0}(N)$ - which is regular away from $\infty$. Such a function exists by Riemann-Roch and a $q$-expansion for one such function can sometimes be computed explicitly using the Dedekind eta-function, as explained in the next subsection.

Using a modular symbol algorithm, one can compute $q$-expansions for a basis of $S_{2}\left(\Gamma_{0}(N)\right)$ consisting of cusp forms with rational Fourier coefficients; cf. for example [Stn]. Write $\omega_{1}, \ldots, \omega_{t}$ for the corresponding holomorphic 1-forms on $X$, where for convenience we denote by $t=\operatorname{dim}_{\mathbf{C}} S_{2}\left(\Gamma_{0}(N)\right)$ the genus of $X$.

Define $\eta_{i}=u \omega_{i}$, which is a differential of the second kind by the residue theorem, and let $\mathcal{B}=\left\{\omega_{1}, \ldots, \omega_{t}, \eta_{1}, \ldots, \eta_{t}\right\} \subset H_{\mathrm{dR}}^{1}(X / \mathbf{Q})$ be the corresponding set of cohomology classes. A simple application of Riemann-Roch shows the following.

Lemma 4.2.1. The set $\mathcal{B}$ is basis for $H_{\mathrm{dR}}^{1}(X / \mathbf{Q})$ whenever $\infty$ is not a Weierstrass point on $X$ and $u$ has a pole of order $t+1$ (i.e., the smallest possible) at $\infty$.

Proof. Since $\infty$ is not a Weierstrass point on $X$, we may assume that $\operatorname{ord}_{\infty}\left(\omega_{i}\right)=$ $i-1$, and thus $\operatorname{ord}_{\infty}\left(\eta_{i}\right)=i-t-2$. For any differential of the second kind $\omega^{\prime}$, we can find a linear combination of $\eta_{1}, \ldots, \eta_{t}$ and $d h$ for an appropriate rational function $h$ having the same principal part as $\omega^{\prime}$. Thus the difference is holomorphic, and lies in the span of $\left\{\omega_{1}, \ldots, \omega_{t}\right\}$. 
Remark 4.2.2. By a result of Ogg [O], the cusp $\infty$ is not a Weierstrass point when the level $N$ is prime or, more generally, when $N=p M$ for prime $p$ and an integer $M \geq 1$ such that $X_{0}(M)$ has genus zero and $p \nmid M$.

When $\infty$ is a Weierstrass point, there is a rational function with a single pole at $\infty$ of order $\leq g(X)$. When $u$ is taken to be such a function, then the set $\mathcal{B}$ will never be a basis. Indeed, since $\infty$ is a Weierstrass point, there exists a holomorphic differential form $\omega$ with order of vanishing $\geq g(X)$ at $\infty$. Then $u \omega$ is still holomorphic, and thus lies in the span of $\left\{\omega_{1}, \ldots, \omega_{t}\right\}$. But $u \omega$ is also in the span of $\left\{\eta_{1}, \ldots, \eta_{t}\right\}$ by definition of the $\eta_{i}$, giving rise to a linear dependence relation. Hence, in order for $\mathcal{B}$ to be a basis, it is necessary for $u$ to have a pole at $\infty$ of order greater than the order of vanishing at $\infty$ of any holomorphic differential.

This lemma is not strictly necessary for the computation, but rather serves to guarantee its success in certain cases. Even if the hypotheses of the lemma do not hold (for example, if $\infty$ is not a Weierstrass point but $u$ has a pole of order $>t+1$ ), the set $\mathcal{B}$ may still be a basis of $H_{\mathrm{dR}}^{1}(X / \mathbf{Q})$, and almost always is at levels $<200$. Moreover, this can be checked easily in any particular example by computing the matrix for the Poincaré pairing.

Given one basis $\mathcal{B}$ for $H_{\mathrm{dR}}^{1}(X / \mathbf{Q})$ - for example, one computed as above it is then a matter of linear algebra to produce a better basis that is adapted to the action of the Hecke algebra. Note that the usual formula for the action of the Hecke algebra $\mathbf{T}_{0}$ on holomorphic modular forms in terms of $q$-expansions extends to weakly holomorphic modular forms (i.e., meromorphic 1-forms on $X$ with possible poles only at the cusps), and preserves the subspace of differentials regular on $Y$. In particular, one can compute the action of $\mathbf{T}_{0}$ on any 1-form representing an element of $H_{\mathrm{dR}}^{1}(X / \mathbf{Q})$. Using $q$-series for the elements of the basis $\mathcal{B}$, we can thus write down the matrix $\left[T_{p}\right] \in \operatorname{Mat}_{2 t \times 2 t}(\mathbf{Q})$ that describes the action of any $T_{p} \in \mathbf{T}_{0}$. After identifying $H_{\mathrm{dR}}^{1}(X / \mathbf{Q}) \approx \mathbf{Q}^{2 t}$ via the basis $\mathcal{B}$, by finding the eigenspaces of finitely many such matrices one can write down $\mathbf{Q}$-bases for each isotypic component of $H_{\mathrm{dR}}^{1}(X / \mathbf{Q})$. As is shown in [Stn], the Hecke algebra $\mathbf{T}_{0}$ is generated as a $\mathbf{Z}$-module by $T_{i}$ for $1 \leq i \leq \frac{m}{6}-\frac{m-1}{N}$, where $m=[\Gamma(1)$ : $\left.\Gamma_{0}(N)\right]$. This gives an upper bound on the number of matrices needed, although in practice considerably fewer are necessary. Using these it is simple linear algebra to produce the desired symplectic basis $\omega_{g, 1}, \ldots, \omega_{g, 2 k}$ for each isotypic component $H_{\mathrm{dR}}^{1}(X / \mathbf{Q})[g]$. Specifically, if $h_{n}(x)$ is the minimal polynomial for $a_{n}(g)$, then compute the intersection of the null spaces of $h_{n}\left(T_{n}\right)$ for different values of $n$ until the resulting space has the correct dimension.

4.3. Modular units and $\eta$-products. The preceding discussion raises the question of how to compute the $q$-expansion about $\infty$ of a rational function $u$ used to write down an initial choice of basis $\mathcal{B}$ for $H_{\mathrm{dR}}^{1}(X / \mathbf{Q})$.

Recall that a modular unit (for $\Gamma_{0}(N)$ ) is a modular function $u \in \mathbf{Q}(X)^{\times}$whose associated divisor is supported on the cusps of $X=X_{0}(N)$. Denote by $U$ the multiplicative group of modular units.

Definition 4.3.1. The eta group $U_{\eta}$ is the subgroup of $\mathbf{Q}(X)^{\times}$of rational functions of the form

$$
u(q)=\lambda \prod_{0<d \mid N} \eta\left(q^{d}\right)^{r_{d}}
$$


where $\lambda \in \mathbf{Q}^{\times}, \eta(q)=q^{1 / 24} \prod_{n>0}\left(1-q^{n}\right)$ is the classical eta function and $\left\{r_{d}\right\}_{d \mid N}$ is a collection of integers satisfying the following conditions:

i. $\sum_{d \mid N} r_{d}=0$,

ii. $\prod_{d \mid N} d^{r_{d}} \in \mathbf{Q}^{\times}$is a square,

iii. $\left(n_{d}\right):=A_{N} \cdot\left(r_{d}\right)$ is a vector (indexed by divisors $d$ of $N$ ) of integers divisible by 24 , where $A_{N}$ is the $\sigma(N) \times \sigma(N)$-matrix whose entry indexed by $\left(d, d^{\prime}\right)$ is $\frac{N \cdot\left(d, d^{\prime}\right)^{2}}{d d^{\prime}\left(d^{\prime}, N / d^{\prime}\right)}$.

Work of Newman and Ligozat (cf., e.g., G]) shows that such functions are indeed modular units on $X$; that is, $U_{\eta} \subset U$. In fact more is true:

Proposition 4.3.2. $\mathbf{Q} \otimes_{\mathbf{Z}} U_{\eta}=\mathbf{Q} \otimes_{\mathbf{Z}} U$.

Proof. It easy to see that the set $\left\{\frac{a}{d}: d \mid N, a \in(\mathbf{Z} /(d, N / d) \mathbf{Z})^{\times}\right\} \subset \mathbf{P}^{1}(\mathbf{Q})$ is a complete set of representatives of the cusps of $X$. If $(d, N / d)=1$, we just take $a=1$.

The subspace $\mathbf{Q} \otimes U_{\eta} \subset \mathbf{Q} \otimes U$ coincides with $\mathbf{Q} \otimes U^{\prime}$, where $U^{\prime} \subset U$ consists of modular units that have the same valuation at any two cusps $a / d, a^{\prime} / d$ with the same denominator; cf. [G, Prop. 2]. This implies the proposition in light of the next lemma, since an element $u \in U \subset \mathbf{Q}(X)$ has the same valuation at any two Galois-conjugate cusps.

Lemma 4.3.3. Let $d \mid N$. Then the cusp $1 / d$ is rational if and only if $(d, N / d)=1$. More generally, the Galois orbit of the cusp $1 / d$ is $\operatorname{Gal}(\overline{\mathbf{Q}} / \mathbf{Q}) \cdot \frac{1}{d}=\left\{\frac{a}{d}: a \in\right.$ $\left.(\mathbf{Z} /(d, N / d) \mathbf{Z})^{\times}\right\}$.

Proof of the lemma. We prove the first statement using the results of [Stv, §1.3]. Namely, it is known that the cusps of $X$ are rational over $\mathbf{Q}\left(\zeta_{N}\right)$, and the Galois action of $\left.\operatorname{Gal}\left(\mathbf{Q}\left(\zeta_{N}\right)\right) / \mathbf{Q}\right) \simeq(\mathbf{Z} / N \mathbf{Z})^{\times}$can be described explicitly as follows [Stv, Thm. 1.3.1]: given $b \in(\mathbf{Z} / N \mathbf{Z})^{\times}$, let $\tau_{b}$ be the automorphism of $\mathbf{Q}\left(\zeta_{N}\right)$ that sends $\zeta_{N} \mapsto \zeta_{N}^{b}$. If $a \in \mathbf{Z}$ is chosen so that $a b \equiv 1(\bmod N)$ then $\tau_{b}$ sends the cusp $\frac{1}{d}$ to $\frac{1}{a d}=\frac{a}{d}$. Hence the Galois orbit of $\frac{1}{d}$ is

$$
\left\{\frac{a}{d}: a \in(\mathbf{Z} / N \mathbf{Z})^{\times}\right\},
$$

and it can be shown by an elementary argument that this set of cusps is equal to the image of

in $\Gamma_{0}(N) \backslash \mathbf{P}^{1}(\mathbf{Q})$.

$$
\left\{\frac{a}{d} \mid a \in(\mathbf{Z} /(d, N / d) \mathbf{Z})^{\times}\right\}
$$

By the Riemann-Roch theorem, there exist nonconstant rational functions on $X$ that are regular away from $\infty$. The proposition implies that an integer power of such a function belongs to the subgroup $U_{\eta} \subset U$, which yields the following.

Corollary 4.3.4. There exists an eta product $u \in U_{\eta}$ that is regular away from $\infty$.

It is thus possible to compute the rational function $u$ required in the computation of a basis for $H_{\mathrm{dR}}^{1}(X)$ as an eta product.

A practical approach to finding the vector $\left(r_{d}\right)_{d \mid N}$ giving rise to the $u$ we seek is to apply a linear programming algorithm: one minimizes the pole order $-n_{N}$ of $u$ at $\infty$ subject to the criteria of Newman-Ligozat in Definition 4.3.1 and the condition that the orders $n_{d}$ of $u$ at other cusps are nonnegative. 
Remark 4.3.5. To determine the complexity of the algorithm described in this paper (see 4.8), it is necessary to bound effectively (as a function of $N$ ) the order of the pole at $\infty$ of the eta quotient $u$ in Corollary 4.3.4. This can be done by examining the proof of Corollary 4.3.4. By the Riemann-Roch theorem, there is a nonconstant rational function $w$ on $X$ that is regular on $Y$ and has a pole of order $\leq t=\operatorname{genus}(X)$ at $\infty$. From the formula for genus $(X)$ as a function on $N$, one can thus extract the bound $-\operatorname{ord}_{\infty}(w)=O(N \log \log N)$; cf. for example CWZ. (We adopt the convention that unless decorated with a subscript, an expression $O(-)$ denotes a bound with an absolute implied constant.) An examination of the proof of [G, Prop. 2], which was invoked to show Proposition 4.3.2, actually gives the more precise result that $w^{\mu}$ belongs to $U_{\eta}$ for an integer $\mu=O\left(\operatorname{det} A_{N}\right)$. Combining this with the explicit formula [G. Prop. 1] for $\operatorname{det} A_{N}$, one deduces the estimate $-\operatorname{ord}_{\infty}(u)=O\left(N^{2 \sigma_{0}(N)+2}\right)$, where $\sigma_{0}(N)$ denotes the number of positive divisors of $N$.

4.4. Computing the Poincaré dual $\gamma_{f}$ of $\omega_{f}$. Assume that $\left\{\gamma^{(j)}\right\}$ is a collection of elements of $\Gamma_{0}(N)$ with small lower-left entries $c N$, whose homology classes $\gamma_{0}^{(j)}$ generate $H_{1}\left(X^{\text {an }}, \mathbf{Z}\right)$. By a brute-force search it is straightforward to find such elements $\gamma^{(j)}$ in practice. (For small $N$, often one only needs to take $c$ no greater than 2 or 3 .)

For any $m \in H_{1}\left(X^{\text {an }}, \mathbf{C}\right)$, write $\eta_{m}$ for the Poincaré dual of $c$; conversely, for any differential $\eta$ of the second kind on $X$, let $m_{\eta} \in H_{1}\left(X^{\text {an }}, \mathbf{C}\right)$ denote the Poincaré dual of its cohomology class. We normalize the Poincaré duality isomorphism so that it is characterized by the property

$$
\left\langle\eta_{m}, \eta\right\rangle=\int_{m} \eta
$$

The vector space $H_{1}\left(X^{\mathrm{an}}, \mathbf{C}\right)$ is also equipped with an intersection product, which is related to the Poincaré pairing by the formula

$$
m \cdot m_{\eta}=\frac{1}{2 \pi i}\left\langle\eta_{m}, \eta\right\rangle .
$$

The homology of $X$ also admits a natural action of the Hecke algebra, compatible with the action on cohomology via Poincaré duality. For any normalized eigenform $f \in S_{2}\left(\Gamma_{0}(N)\right)$ and any $m \in H_{1}\left(X^{\text {an }}, \mathbf{C}\right)$, write $m^{f} \in H_{1}\left(X^{\text {an }}, \mathbf{C}\right)[f]$ for the projection of $m$ onto the $f$-isotypic component of homology. Similarly, for $\eta \in$ $H_{\mathrm{dR}}^{1}(X / \mathbf{Q})$ write $\eta^{f}$ for its projection onto the $f$-isotypic component.

We can assume that via the method described above a symplectic basis

$$
\mathcal{S}=\left\{\omega_{f, 1}, \ldots, \omega_{f, \ell}, \eta_{f, 1}, \ldots, \eta_{f, \ell}\right\}
$$

for $H_{\mathrm{dR}}^{1}(X / \mathbf{Q})[f]$ has already been computed, where $\left\langle\omega_{f, i}, \eta_{f, j}\right\rangle=\delta_{i, j}$.

Lemma 4.4.1. Fix $\gamma_{1}, \gamma_{2} \in \Gamma_{0}(N)$ and let $m_{1}, m_{2} \in H_{1}\left(X^{\text {an }}, \mathbf{Z}\right)$ denote the corresponding homology classes on $X$. For any normalized eigenform $f \in S_{2}\left(\Gamma_{0}(N)\right)$, we have

$$
m_{1}^{f} \cdot m_{2}^{f}=\frac{1}{2 \pi i} \sum_{i=1}^{\ell} I\left(\omega_{f, i} ; m_{1}\right) I\left(\eta_{f, i} ; m_{2}\right)-I\left(\omega_{f, i} ; m_{2}\right) I\left(\eta_{f, i} ; m_{1}\right),
$$

where $\omega_{f}=2 \pi i f(z) \mathrm{d} z$ is the 1-form corresponding to $f$. 
Proof. Let $\eta_{k}=\eta_{m_{k}}$ and write $\eta_{k}^{f}=\sum c_{i}^{(k)} \omega_{f, i}+\sum d_{i}^{(k)} \eta_{f, i}$. Then we compute $m_{1}^{f} \cdot m_{2}^{f}=\frac{1}{2 \pi i}\left\langle\eta_{1}^{f}, \eta_{2}^{f}\right\rangle=\sum_{i} \frac{1}{2 \pi i}\left(c_{i}^{(1)} d_{i}^{(2)}-c_{i}^{(2)} d_{i}^{(1)}\right)=\frac{1}{2 \pi i} \sum_{i}\left(I\left(\omega_{f, i} ; \gamma_{1}\right) I\left(\eta_{f, i}, \gamma_{2}\right)-\right.$ $\left.I\left(\eta_{f, i} ; \gamma_{1}\right) I\left(\omega_{f, i} ; \gamma_{2}\right)\right)$.

Using (4.4.1), (4.4.2), and Lemma 4.4.1 we can compute the Poincaré dual $\gamma_{f}$ of $\omega_{f}$. Let $m_{1}, \ldots, m_{2 \ell}$ be modular symbols giving rise to a basis of $H_{1}\left(X^{\text {an }}, \mathbf{Z}\right)[f]$, which can be computed using a modular symbols algorithm (cf. Stn]). In particular, if $f$ is new, then $\ell=1$. Write $A$ for the matrix $\left(m_{i} \cdot m_{j}\right)_{1 \leq i, j \leq 2 \ell}$, which can be computed using Lemma 4.4.1, and let $v$ be the column vector $\left(m_{i} \cdot m_{\omega_{f}}\right)_{i=1}^{2 \ell}$, which can be computed using (4.4.2) in conjunction with (4.4.1). Then the vector $A^{-1} v$ gives the coefficients expressing $m_{\omega_{f}}$ as a linear combination of $m_{1}, \ldots, m_{2 \ell}$. These coefficients can then easily be converted to an expression for $\gamma_{f}$ as a linear combination of $\left\{\gamma_{0}^{(j)}\right\}$.

4.5. Computing the adjustments $\int_{\gamma_{f}} \alpha$. Write the homology class $\gamma_{f}$ Poincaré dual to $\omega_{f}$ as

$$
\gamma_{f}=\sum \beta_{j} \gamma_{0}^{(j)}
$$

for $\beta_{j} \in \mathbf{C}$ and homology classes $\gamma_{0}^{(j)}$ whose lifts $\gamma^{(j)}$ to $\Gamma_{0}(N)$ have small lower-left entries $c N$. Let $\omega$ and $\eta$ be differentials of the second kind, at least one of which is regular at $\infty$. Using the methods described so far, we are already able to compute

$$
z_{\omega, \eta}:=\sum_{j} \beta_{j} \int_{\tau_{0}}^{\gamma^{(j)} \tau_{0}} \omega F_{\eta} .
$$

We stress that the value of $z_{\omega, \eta}$ depends on $\tau_{0}$ and the choices we made in representing $\gamma_{f}$. It is simply one part of the iterated integral $J_{\omega, \eta}\left(\gamma_{f}\right)=\int_{\gamma_{f}} \omega \cdot \eta-\alpha_{\omega, \eta}$, which is independent of these choices. In this section, we describe a method for computing $J_{\omega, \eta}\left(\gamma_{f}\right)-z_{\omega, \eta}$. This amounts to computing the $q$-expansion of $\alpha_{\omega, \eta}$, for then we have

$$
J_{\omega, \eta}\left(\gamma_{f}\right)-z_{\omega, \eta}=-\sum_{j} \beta_{j} \int_{\tau_{0}}^{\gamma^{(j)} \tau_{0}} \alpha_{\omega, \eta} .
$$

Recall that the defining property of $\alpha_{\omega, \eta}$ is that its principal part at $\infty$ agrees with that of $\omega F_{\eta}$ on $\tilde{X}$, modulo $\mathrm{d} q / q$; i.e., their difference has at worst logarithmic poles. However, note that since $\int_{\gamma_{f}} \lambda=0$ for exact 1 -forms $\lambda$, we may replace $\alpha_{\omega, \eta}$ by any cohomologous 1 -form. The cohomology class of $\alpha_{\omega, \eta}$ is determined by the data $\left\langle\lambda_{i}, \alpha_{\omega, \eta}\right\rangle$, where $\lambda_{1}, \ldots, \lambda_{2 t}$ (for $t$ the genus of $X$ ) form a basis of $H_{\mathrm{dR}}^{1}(X / \mathbf{Q}$ ), so it suffices to compute these values of the Poincaré pairing.

We can choose $\lambda_{1}, \ldots, \lambda_{t}$ to be holomorphic. In this case, we can compute

$$
\left\langle\lambda_{i}, \alpha_{\omega, \eta}\right\rangle=\operatorname{res}_{\infty}\left(F_{\lambda_{i}} \cdot \alpha_{\omega, \eta}\right)=\operatorname{res}_{\tilde{\infty}}\left(F_{\lambda_{i}} \cdot F_{\eta} \cdot \omega\right),
$$

where the second equality holds because $\operatorname{res}_{\infty}\left(F_{\lambda_{i}} \cdot \alpha_{\omega, \eta}\right)$ depends only on

$$
\operatorname{pp}_{\infty}\left(\alpha_{\omega, \eta}\right) \quad \bmod \frac{\mathrm{d} q}{q}=\operatorname{pp}_{\tilde{\infty}}\left(\omega F_{\eta}\right) .
$$

Lemma 4.5.1. Let $\lambda_{1}, \ldots, \lambda_{t} \in H^{0}\left(X, \Omega_{X / \mathbf{Q}}^{1}\right)$ be a basis of regular 1-forms on $X$. Then $\alpha \in H_{\mathrm{dR}}^{1}(X / \mathbf{Q})$ lies in the subspace $H^{0}\left(X, \Omega_{X / \mathbf{Q}}^{1}\right)$ if and only if $\left\langle\lambda_{i}, \alpha\right\rangle=0$ for all $1 \leq i \leq t$. 
Proof. The subspace $H^{0}\left(X, \Omega_{X / \mathbf{Q}}^{1}\right) \subseteq H_{\mathrm{dR}}^{1}(X)$ is isotropic for the Poincaré pairing because the pairing can be computed using residues. For dimension reasons, it is maximal isotropic, and the lemma follows.

By the lemma, if $\left\langle\lambda_{i}, \alpha\right\rangle=\left\langle\lambda_{i}, \alpha^{\prime}\right\rangle$ for $i=1, \ldots, t$, then $\alpha-\alpha^{\prime}$ is cohomologous to a regular 1-form. Since $\alpha_{\omega, \eta}$ is only well-defined modulo $H^{0}\left(X, \Omega_{X / \mathbf{Q}}^{1}\right)$, it follows that we can choose $\left\langle\lambda_{i}, \alpha_{\omega, \eta}\right\rangle$ for $i=t+1, \ldots, 2 t$ arbitrarily. For convenience, we choose $\left\langle\lambda_{i}, \alpha_{\omega, \eta}\right\rangle=0$ for $i=t+1, \ldots, 2 t$. Define the matrix $B=\left(\left\langle\lambda_{i}, \lambda_{j}\right\rangle\right)_{i, j=1}^{2 t}$ and the vector

$$
w=\left(\left\langle\lambda_{i}, \alpha_{\omega, \eta}\right\rangle\right)_{i=1}^{2 t}=(\operatorname{res}_{\tilde{\infty}}\left(F_{\lambda_{1}} F_{\eta} \omega\right), \cdots, \operatorname{res}_{\tilde{\infty}}\left(F_{\lambda_{t}} F_{\eta} \omega\right), \overbrace{0, \ldots, 0}^{t}) .
$$

It then follows by elementary linear algebra that the vector $B^{-1} w$ yields the coefficients of an expression for $\alpha_{\omega, \eta}$ as a linear combination of $\lambda_{1}, \ldots, \lambda_{2 t}$.

4.6. Computing the coefficients $c_{i j}$. Using the methods described so far, we may compute all the integrals $\int_{\gamma_{f}}\left(\omega \cdot \eta-\alpha_{\omega, \eta}\right)$ occurring in (3.2.2). The last input needed to evaluate (3.2.2) is the set of coefficients $c_{i j}^{n}$ appearing in that formula. Using Lemma 3.2.1, these coefficients can be computed using the matrix $A_{n}$ which expresses the right action of $T_{n}$ on $H_{\mathrm{dR}}^{1}(X / \mathbf{Q})[g]$ with respect to the basis $\omega_{g, 1}, \ldots, \omega_{g, 2 k}$, and the matrix $B=\left(\left\langle\omega_{g, i}, \omega_{g, j}\right\rangle\right)_{1 \leq i, j<2 k}$, which by our choice of basis is the standard symplectic matrix. In section 4.2 , we gave a method for computing $A_{n}$ using the action of $T_{n}$ on $q$-expansions. However, this only works if $\operatorname{gcd}(n, N)=1$, so we must resort to other methods. We exploit the fact that the action of $T_{n}$ on $H_{1}\left(X^{\text {an }}, \mathbf{C}\right)[g]$ is readily computable using modular symbols; see Stn] for details. Recall that we have a Hecke-equivariant duality

$$
H_{\mathrm{dR}}^{1}\left(X^{\mathrm{an}}, \mathbf{C}\right)[g] \times H_{1}\left(X^{\mathrm{an}}, \mathbf{C}\right)[g] \rightarrow \mathbf{C}
$$

given by the integration pairing $\langle\omega, \lambda\rangle=\int_{\gamma} \lambda$; here the Hecke-equivariance means that $\left\langle T_{n} \omega, \alpha\right\rangle=\left\langle\omega, T_{n} \alpha\right\rangle$. Using modular symbols and the techniques of Stn] one can compute the matrix $B_{n}$ of $T_{n}$ acting on $H_{1}\left(X^{\text {an }}, \mathbf{C}\right)[g]$ on the left, with respect to a basis $m_{1}, \ldots, m_{2 k}$. Write $Q=\left(\left\langle\omega_{i}, m_{j}\right\rangle\right)_{i, j=1}^{2 k}$, which can be computed efficiently via the method explained in $\$ 4.1$ (using an appropriate basis $\left\{m_{j}\right\}$ derived from the generators $\gamma_{0}^{(j)}$ for $H_{1}\left(X^{\text {an }}, \mathbf{Z}\right)$ discussed above). Then it is straightforward linear algebra to show that $A_{n}=Q B_{n} Q^{-1}$.

4.7. Computing the denominator $d_{g, n}$. The final ingredient to be computed is the denominator $d_{g, n}$, or the smallest positive integer such that $d_{g, n} T_{g, n} \in \mathbf{T}_{\mathbf{Z}}$. This can be accomplished by computing a $\mathbf{Z}$-basis for the ( $\mathbf{Z}$-finite free) Hecke algebra $\mathbf{T}_{\mathbf{Z}}$ as a subring of $M_{2 t}(\mathbf{Q})$, where $t$ is the genus of $X_{0}(N)$, by identifying $\mathbf{T}$ with an algebra of endomorphisms of the $(2 t)$-dimensional $\mathbf{Q}$-vector space of cuspidal modular symbols of weight 2 and level $N$. As $\mathbf{T}_{\mathbf{Z}}$ is generated as an abelian group by $T_{i}$ for $1 \leq i \leq \frac{m}{6}-\frac{m-1}{N}$, where $m=\left[\Gamma(1): \Gamma_{0}(N)\right]$, this is a finite computation. Once $\mathbf{T}_{\mathbf{Z}}$ has been computed it is a simple matter to find the matrix representation of $T_{g, n}$ and compute the smallest $d_{g, n}$ such that $d_{g, n} T_{g, n} \in \mathbf{T}_{\mathbf{Z}}$.

For more details on modular symbols and generating the Hecke algebra, see [Stn]. 
4.8. Remarks on complexity. The complexity of the computations we have described is primarily determined by the number $n_{D}$ of Fourier coefficients required to compute $J_{\omega, \alpha}\left(\gamma_{f}\right)$ to a given number $D$ of digits of accuracy. In this subsection we sketch a method for obtaining a bound on $n_{D}$ in terms of $N$.

4.8.1. Write the Fourier expansion of $u$ as

$$
u(\tau)=\sum_{n \geq-n_{0}} c_{n} q^{n}, \quad q=e^{2 \pi i \tau}, \tau \in \mathfrak{H} .
$$

Let the principal part of $u$ at $\infty$ be

$$
\operatorname{pp}_{\infty}(u)=\sum_{-n_{0} \leq-m \leq 0} \frac{d_{m}}{m} q^{-m}, \quad d_{m}=m c_{-m} .
$$

In [BO, Bringmann and Ono prove an exact formula for the Fourier coefficients of harmonic Maass forms, of which weakly holomorphic modular functions such as $u$ are examples. To avoid introducing unnecessary notation, we state only the very special case of their result applicable to our situation. We remark that long ago Rademacher used the circle method to prove a similar exact formula for the coefficients of the $j$-function $[\mathrm{R}$, and a modification of his argument would probably yield a simpler and more direct proof of the special case we require. Using $\mathrm{BO}$, Thm. 1.1], one can express the coefficients $c_{n}, n>0$ in terms of the coefficents $d_{m}$, the order-1 $I$-Bessel function $I_{1}(z)$, and the Kloosterman sum

$$
K(-m, n, c):=\sum_{\substack{0<v<c \\(v, c)=1}} \exp \left(\frac{2 \pi i}{c}(n v+m \bar{v})\right)
$$

where $\bar{v}$ denotes the multiplicative inverse of $v$ modulo $c$. Namely, loc. cit. yields the formula

$$
c_{n}=2 \pi \sum_{-n_{0} \leq-m \leq 0} d_{m} \sum_{\substack{c>0 \\ c \equiv 0}}\left(\frac{m}{n}\right)^{1 / 2} \frac{K_{0}(-m, n, c)}{c} I_{1}\left(\frac{4 \pi \sqrt{|m n|}}{c}\right), \quad n>0 .
$$

By Remark 4.3.5, we have

$$
n_{0}=-\operatorname{ord}_{\infty}(u) \leq A_{1} N^{2 \sigma_{0}(N)+2},
$$

where the constant $A_{1}$ is absolute and $\sigma_{0}$ denotes the divisor function. We can trivially bound the numbers $d_{m}$ as follows. Let $\xi_{r}(x)=r e^{2 \pi i x}$ for $0<r<1$ and set $y=-\frac{1}{2 \pi} \log r>0$. The Cauchy integral formula applied to the meromorphic function $U(q)=\sum c_{n} q^{n}$ of $q$ in the unit disk gives

$$
\frac{d_{m}}{m}=\frac{1}{2 \pi i} \int_{\xi_{r}} \frac{U(q)}{q^{m+1}} \mathrm{~d} q=e^{2 \pi m y} \int_{0}^{1} u(x+i y) e^{-2 \pi i n x} \mathrm{~d} x .
$$

Taking $y=1$, say, we thus have

$$
\left|d_{m}\right| \leq m e^{2 \pi m} \int_{0}^{1}|u(x+i)| \mathrm{d} x=m e^{2 \pi i m} \int_{0}^{1} \prod_{d \mid N}|\eta(d x+i d)|^{r_{d}} \mathrm{~d} x,
$$

where $\eta$ is the Dedekind eta function and $\left(r_{d}\right)_{d \mid N}$ is the vector giving rise to $u$. Recall that $\eta(z)$ is a nonvanishing holomorphic function on $\mathfrak{H}$, and satisfies $|\eta(z+1)|=$ 
$|\eta(z)|$. Thus there is an absolute upper bound $B$ for $\max \left(|\eta(z)|,|\eta(z)|^{-1}\right)$ on the strip $\{1-\epsilon \leq \operatorname{Im}(z) \leq N+\epsilon\} \subset \mathfrak{H}$. It follows that

$$
\left|d_{m}\right| \leq m e^{2 \pi m} B^{\sum\left|r_{d}\right|} .
$$

To control this quantity we must bound both $B$ and $\sum\left|r_{d}\right|$ in terms of $N$.

In regard to $B$, note that since $\eta(z)$ is holomorphic on $\mathfrak{H}^{*}$, the upper bound for $|\eta(z)|$ on the strip of interest poses no problem; it is a constant independent of $N$. To find a lower bound for $|\eta(z)|$ on our strip, we can make use of the Euler formula

$$
\eta(z)=e^{\pi i z / 12}\left(1+\sum_{m \geq 1} q^{\frac{3 m^{2}-m}{2}}+q^{\frac{3 m^{2}+m}{2}}\right), \quad q=e^{2 \pi i z} .
$$

From this it is straightforward to deduce a bound $|\eta(z)|^{-1}=O\left(e^{\pi N / 12}\right)$, for $z$ in the strip in question.

To handle $\sum\left|r_{d}\right|$, we recall some results from numerical analysis. Let $A$ be an invertible $\sigma \times \sigma$ matrix of real numbers. We denote by $\|A\|_{2}$ the quantity $\sup _{x \in \mathbf{R}^{\sigma},|x|=1}|A x|$ and by $\|A\|_{\text {max }}$ the maximum of the absolute values of the matrix entries $a_{i j}$. A result in numerical linear algebra [Mal, Thm. 2.1] asserts that when $A$ has integer entries, one can control its so-called "condition number" and obtain the estimate

$$
\left\|A^{-1}\right\|_{2} \leq \sigma^{2+\sigma / 2}\|A\|_{\max }^{\sigma-1} .
$$

We apply this result to the matrix $A_{N}$ from Definition 4.3.1 with $\sigma=\sigma_{0}(N)$. By Newman and Ligozat's result, the vector $\mathbf{r}=\left(r_{d}\right)_{d \mid N}$ and the vector $\mathbf{n}=$ $\left(24 \operatorname{ord}_{a / d}(u)\right)_{d \mid N}$ (where $a / d$ is any cusp of $X_{0}(N)$ of denominator $d$ ) satisfy $\mathbf{r}=$ $A_{N}^{-1} \mathbf{n}$. Moreover, since $u$ has a pole of order $n_{0}$ at infinity and no other poles, the fact that $\div(u)$ has degree 0 implies $|\mathbf{n}|=O\left(n_{0} \sqrt{\sigma_{0}(N)}\right)$. Finally, the formula for the entry of $A_{N}$ indexed by $\left(d, d^{\prime}\right)$ entails $\left\|A_{N}\right\|_{\max } \leq N^{3}$. We thus have, using (4.8.2),

$$
\begin{aligned}
\sqrt{\sum_{d \mid N}\left|r_{d}\right|} & \leq \sqrt{\sum_{d \mid N}\left|r_{d}\right|^{2}}=|\mathbf{r}| \leq \| A_{N}^{-1}||_{2}|\mathbf{n}| \\
& \leq \sigma_{0}(N)^{\frac{\sigma_{0}(N)+5}{2}} n_{0} N^{3\left(\sigma_{0}(N)-1\right)}=O\left(N^{\frac{11 \sigma_{0}(N)+1}{2}}\right) .
\end{aligned}
$$

Consequently, for an absolute constant $C$ we have

$$
\begin{aligned}
\left|d_{m}\right| & \ll m e^{2 \pi m} e^{\pi N \sum\left|r_{d}\right| / 12} \leq m e^{2 \pi m} e^{C N^{11 \sigma_{0}(N)+2}} \\
& \leq n_{0} e^{2 \pi n_{0}} e^{C N^{11 \sigma_{0}(N)+2}} \\
& =O\left(n_{0} \exp \left(2 \pi N^{2 \sigma_{0}(N)+2}+C N^{11 \sigma_{0}(N)+2}\right)\right) .
\end{aligned}
$$

From this we deduce

$$
\left|d_{m}\right|=O\left(n_{0} \exp \left(A_{0} N^{11 \sigma_{0}(N)+2}\right)\right),
$$

for an absolute constant $A_{0}$.

From (4.8.1) and (4.8.3), standard estimates for Kloosterman sums, and asymptotics for the $I$-Bessel function, one obtains by the method of [BrPh, $\S \S 5.1-5.2]$ the estimate

$$
c_{n}=O\left(N^{5 / 4} n_{0}^{7 / 4} n^{-3 / 4} \exp \left(A_{0} N^{11 \sigma_{0}(N)+2}+N^{-1} 4 \pi \sqrt{n n_{0}}\right)\right) .
$$


In light of (4.8.2) this yields

$$
c_{n}=O\left(n^{-3 / 4} \exp \left(A_{2} N^{11 \sigma_{0}(N)+2}+\frac{4 \pi \sqrt{A_{1}}}{N} N^{\sigma_{0}(N)+1} \sqrt{n}\right)\right),
$$

for absolute constants $A_{1}, A_{2}$.

4.8.2. The coefficients $c_{n}$ determine the Fourier coefficients of the 1-forms occurring in the formula (3.2.2) for $P_{g, f, n}$. Unfortunately the relationship is indirect, as the construction of the 1 -forms $\omega_{g, i}$ and $\alpha_{\omega_{g, i}, \omega_{g, j}}$ involves multiplying $u$ against a basis of cusp forms for $\Gamma_{0}(N)$ and then performing a lot of linear algebra. By the Ramanujan-Petersson conjecture (which in this context is a theorem of Eichler [E]), the cusp forms have $n$th coefficient of size $O_{\delta}\left(n^{\frac{1}{2}+\delta}\right)$. It follows that $n$th Fourier coefficient of an element of the basis $\mathcal{B}$ for $H_{\mathrm{dR}}^{1}(X / \mathbf{Q})$ computed in 4.2 has size at most

$$
O\left(P(n) \exp \left(A_{2} N^{11 \sigma_{0}(N)+2}+\frac{4 \pi \sqrt{A_{1}}}{N} N^{\sigma_{0}(N)+1} \sqrt{n}\right)\right),
$$

for absolute constants $A_{1}, A_{2}$ and a universal polynomial $P(n)$. To compute the 1-forms $\omega_{g, i}$ and $\alpha_{\omega_{g, i}, \omega_{g, j}}$, linear algebra operations are performed on this basis, which spans a vector space of dimension genus $(X)=O(N \log \log N)$. It thus seems likely that a careful analysis of the linear algebra operations performed would yield a bound

$$
O\left(Q(n) \exp \left(A_{3} N^{11 \sigma_{0}(N)+2}+\frac{4 \pi \sqrt{A_{1}}}{N} N^{\sigma_{0}(N)+1} \sqrt{n}\right)\right),
$$

for the $n$th Fourier coefficient of any 1-form integrated in the course of computing (3.2.2). Here $A_{1}, A_{3}$ are absolute constants and $Q(X)$ is a universal polynomial independent of $N$.

Suppose $\lambda$ is such a 1 -form (on $X$ or $\tilde{X}$ ), and consider the problem of integrating the pullback of $\lambda$ to $\mathfrak{H}^{*}$ along a path from $\tau_{1}$ to $\tau_{2}$. By the method explained in 44.1. we can assume that $\operatorname{im} \tau_{1}, \operatorname{im} \tau_{2} \geq\left(c_{N}^{*} \cdot N\right)^{-1}$, where $c_{N}^{*} \cdot N$ is the largest of the lower left entries of the elements $\gamma^{(j)} \in \Gamma_{0}(N)$ introduced at the beginning of 4.4. Recall that these consisted of a collection of elements that span $H_{1}\left(X^{\text {an }}, \mathbf{Z}\right)$ and have lower left entries as small as possible. We do not know how to bound $c_{N}^{*}$ in terms of $N$, although in practice it seems to be very small.

If the Laurent expansion for $\lambda$ about $\infty$ (or a lift of $\infty$ to $\tilde{X}$ ) is $\lambda=\sum a_{\lambda}(n) \frac{\mathrm{d} q}{q}$, then setting $\tau_{j}=x_{j}+i y_{j}$ for $j=1,2$ (where $\left.y_{j} \geq\left(c_{N}^{*} N\right)^{-1}\right)$, we have

$$
\int_{\tau_{1}}^{\tau_{2}} \lambda=\sum_{n \gg-\infty} \frac{a_{\lambda}(n)}{n}\left(e^{2 \pi i n x_{2}} e^{-2 \pi n y_{2}}-e^{2 \pi i n x_{1}} e^{-2 \pi n y_{1}}\right) .
$$

Our problem is to determine $n_{D}$ such that the tails of these sums are bounded by the requisite precision, say $10^{-D}$. It clearly suffices to take for $n_{D}$ any $m$ such that

$$
S(m):=\sum_{n \geq m} n^{-1}\left|a_{\lambda}(n)\right| e^{-2 \pi n y} \leq 10^{-D} .
$$

Granting (4.8.5), we have

$$
S(m) \ll \sum_{n \geq m} n^{-1} Q(n) \exp \left(A_{3} N^{11 \sigma_{0}(N)+2}+\frac{4 \pi \sqrt{A_{1}}}{N} N^{\sigma_{0}(N)+1} \sqrt{n}-\frac{2 \pi n}{N c_{N}^{*}}\right) .
$$

For a suitable constant $A_{4}$, we can assume

$$
n^{-1} Q(n) \exp \left(\frac{4 \pi \sqrt{A_{1}}}{N} N^{\sigma_{0}(N)+1} \sqrt{n}\right) \leq \exp \left(\frac{A_{4}}{N} N^{\sigma_{0}(N)+1} \sqrt{n}\right) .
$$


Thus

$$
\begin{aligned}
S(m) & \ll \exp \left(A_{3} N^{11 \sigma_{0}(N)+2}\right) \sum_{n \geq m} \exp \left(\frac{1}{N c_{N}^{*}}\left(A_{4} c_{N}^{*} N^{\sigma_{0}(N)+1} \sqrt{n}-2 \pi n\right)\right) \\
& \leq \exp \left(A_{3} N^{11 \sigma_{0}(N)+2}\right) \sum_{n \geq m} e^{-n / N c_{N}^{*}}=\exp \left(A_{3} N^{11 \sigma_{0}(N)+2}\right) \frac{e^{-m / N c_{N}^{*}}}{1-e^{-1 / N c_{N}^{*}}},
\end{aligned}
$$

provided $m$ is large enough that $A_{4} c_{N}^{*} N^{\sigma_{0}(N)+1} \sqrt{n}-2 \pi n \leq-n$ for $n \geq m$. The latter can be ensured by requiring $m \geq A_{5} c_{N}^{* 2} N^{2 \sigma_{0}(N)+2}$ for an absolute constant $A_{5}$. It follows that, provided (4.8.5) holds, we have the following estimate for $n_{D}$ in terms of $D$ and $N$ :

$$
n_{D}=O\left(\max \left\{N c_{N}^{*}\left(D+N^{11 \sigma_{0}(N)+2}\right), c_{N}^{* 2} N^{2 \sigma_{0}(N)+2}\right\}\right),
$$

where the implied constant is absolute, as always.

\section{Numerical EXAMPLES}

5.1. Example: 37a1. Take $N=37$ in the setup of our algorithm. In this setting, the space of regular differentials on $X=X_{0}(37)$ is spanned by $\omega_{f}$ and $\omega_{g}$, which are associated to elliptic curves over $\mathbf{Q}$ (labeled $37 \mathbf{a} 1$ and $37 \mathrm{~b} \mathbf{1}$ in Cremona's database) of ranks 1 and 0 , respectively. The elliptic curve $37 a 1$ has minimal Weierstrass equation given by

$$
37 a 1: y^{2}+y=x^{3}-x,
$$

and its Mordell-Weil group is generated by the point $(0,0)$.

By computing the periods attached to $\omega_{f}$ and $\omega_{g}$, it can be checked that the classes of the matrices

$$
\gamma_{1}=\left(\begin{array}{cc}
2 & -1 \\
37 & -18
\end{array}\right), \gamma_{2}=\left(\begin{array}{cc}
3 & -1 \\
37 & -12
\end{array}\right), \gamma_{3}=\left(\begin{array}{cc}
5 & 2 \\
37 & 15
\end{array}\right), \gamma_{4}=\left(\begin{array}{ll}
14 & 3 \\
37 & 8
\end{array}\right)
$$

generate the rational homology of $X$. These are a "nice" basis for the homology in the sense of the first paragraph of 4.4 , that is, the lower left entries are exactly 37 (rather than $37 c$ for $|c|>1$ ), so the integral $\int_{\tau}^{\gamma_{i} \tau} \lambda$ can be evaluated efficiently for any meromorphic differential 1-form $\lambda$ on $X_{0}(37)$ or its universal cover regular away form $\infty$, by the method of 4.1 .

To obtain differentials of the second kind representing classes in the de Rham cohomology, we consider the elements of the form

$$
\eta_{1}=u \cdot \omega_{f}, \quad \eta_{2}=u \cdot \omega_{g}, \quad u=\eta(q)^{2} \eta\left(q^{37}\right)^{-2}=q^{-3} \prod_{n=1}^{\infty}\left(1-q^{n}\right)^{2}\left(1-q^{37 n}\right)^{-2},
$$

where $\eta(q)$ is the Dedekind eta function. The modular function $u$ is an example of an eta product with its only pole at $\infty$, as considered in $\$ 4.3$. It is not hard to check directly (by calculating the Poincaré pairing on all pairs of elements) that the classes of $\omega_{f}, \omega_{g}, \eta_{1}$ and $\eta_{2}$ generate the de Rham cohomology of $X$; alternatively, one could apply Lemma 4.2.1 
After computing the matrix $M$ of the Hecke operator $T_{2}$ acting on $H_{\mathrm{dR}}^{1}\left(X_{0}(37)\right)$ with respect to the basis $\omega_{f}, \omega_{g}, \eta_{1}, \eta_{2}$, and then determining the eigenspaces of $M$, one finds that

$$
\begin{aligned}
\eta_{f} & =\frac{1}{4}\left(-37 \omega_{g}+4 \eta_{1}-8 \eta_{2}\right) \\
\eta_{g} & =\frac{1}{4}\left(37 \omega_{f}-6 \eta_{1}+10 \eta_{2}\right)
\end{aligned}
$$

are in the $f$ and $g$ isotypic components of the de Rham cohomology respectively. In addition these linear combinations of 1 -forms have been chosen so that $\left\{\omega_{f}, \eta_{f}\right\}$ and $\left\{\omega_{g}, \eta_{g}\right\}$ form symplectic bases for the components with respect to the Poincaré pairing.

When one computes the Poincaré dual $\gamma_{f}$ of $\omega_{f}$ as in 4.4 one finds (with our normalization) that it is

$$
\gamma_{f}=\frac{1}{2 \pi i}\left(A\left(\left[\gamma_{2}\right]-\left[\gamma_{3}\right]+\left[\gamma_{4}\right]\right)-B\left(-\left[\gamma_{1}\right]+2\left[\gamma_{2}\right]\right)\right) .
$$

Here

$$
A \approx(2.4513893 \ldots) i, \quad B \approx 2.9934586 \ldots
$$

are certain linear combinations of the periods of $f$ against a basis of $H_{1}(X)[f]$; see 4.4 for a more exact description.

The method of 4.5 can be used to compute $\alpha_{\omega_{g}, \eta_{g}}$. However, in this case, it is easy to find $\alpha_{\omega_{g}, \eta_{g}}$ by inspection. Working with principal parts, one finds that $\operatorname{pp}_{\infty}\left(\omega_{g} F_{\eta_{g}}\right) \equiv \operatorname{pp}_{\infty}\left(\frac{1}{4}\left(\eta_{1}-\eta_{2}\right)\right) \bmod \frac{\mathrm{d} q}{q}$. Thus we may take $\alpha_{\omega_{g}, \eta_{g}}=\frac{1}{4}\left(\eta_{1}-\eta_{2}\right)$. Integrating this over $\gamma_{f}$ yields the rational number $\int_{\gamma_{f}} \alpha_{\omega_{g}, \eta_{g}}=-\frac{1}{2}$ (to many digits of precision).

Since $g$ is a rational newform, then by Remark 3.1.3. we can find all the points $P_{g, f, n}$ by only computing $P_{g, f}$. According to Remark 3.2.2, this amounts to computing the complex number $z_{g, f}:=\int_{\gamma_{f}}\left(\omega_{g} \cdot \eta_{g}-\eta_{g} \cdot \omega_{g}-2 \alpha_{\omega_{g}, \eta_{g}}\right)$. The method in 4.1, coupled with the previous paragraph, yields

$$
z_{g, f}=-0.4093610 \ldots+(1.2256946 \ldots) i .
$$

Let $W$ be the Weierstrass uniformization of $E$. Then the point $W\left(z_{g, f}\right) \in E(\mathbf{C})$ does not necessarily lie in $E(\mathbf{Q})$. This is because $T_{g}$ is a rational combination of cycles, and so $W\left(z_{g, f}\right)$ is a $\mathbf{Q}$-linear combination of points in $E(\mathbf{Q})$. Thus, the image of $W\left(z_{g, f}\right)$ in $E(\mathbf{C}) \otimes \mathbf{Q}$ lies in the subspace $E(\mathbf{Q}) \otimes \mathbf{Q}$. So in order to write $P_{g, f}$ as an element of this space, we must compute the "denominator" of $T_{g}$. As in $\$ 4.7$ one can compute using the first few Fourier coefficients of $f$ and $g$ that the idempotent $e=(0,1) \in \mathbf{Q} \times \mathbf{Q} \simeq \mathbf{T}(\star)$ does not belong to $\mathbf{T}_{\mathbf{Z}} \subset \mathbf{T}$ but $2 e$ does. Here, the identification $(\star)$ associates $T_{n} \otimes 1 \in \mathbf{T}$ to $\left(a_{n}(f), a_{n}(g)\right) \in \mathbf{Q} \times \mathbf{Q}$. By definition, $T_{g}$ corresponds to $e$ as an element of the Hecke algebra, so it's denominator is 2 . Thus, we can write $P_{g, f}=W\left(2 z_{g, f}\right) \otimes \frac{1}{2} \in E(\mathbf{Q}) \otimes \mathbf{Q}$. One finds that $W\left(2 z_{g, f}\right)$ agrees with the global point $\left(\frac{1357}{841}: \frac{28888}{24389}: 1\right)$ to within 13 digits of accuracy using 350 Fourier coefficients, so we are led to conclude that

$$
P_{g, f}=\left(\frac{1357}{841}: \frac{28888}{24389}: 1\right) \otimes \frac{1}{2}=12(0: 0: 1) \otimes \frac{1}{2}=6(0: 0: 1) \in E(\mathbf{Q}) \otimes \mathbf{Q}
$$


5.2. Example: 43a1. Let $N=43$ and let $E$ be the elliptic curve labeled 43a1 in Cremona's database. The modular curve $X=X_{0}(43)$ has genus 3 . There are two isotypic components of $H_{\mathrm{dR}}^{1}(X)$, one of dimension 2 corresponding to the modular form $f$ that parametrized $E$, and another of dimension 4 corresponding to a newform $g$ with Fourier coefficients in $\mathbf{Q}(\sqrt{2})$, associated to an abelian surface quotient of $J_{0}(43)$.

In this case, a linear programming algorithm identifies the eta quotient $u$ that is modular for $\Gamma_{0}(43)$ of weight 0 , holomorphic away from the cusp $\infty$, and with minimal pole order at $\infty$, as

$u=\frac{\eta(q)^{4}}{\eta\left(q^{43}\right)^{4}}=q^{-7}-4 q^{-6}+2 q^{-5}+8 q^{-4}-5 q^{-3}-4 q^{-2}-10 q^{-1}+8+9 q+14 q^{3}+O\left(q^{4}\right)$.

Computing the residue pairing shows that for a basis of cusp forms with rational Fourier coefficients, corresponding to holomorphic 1-forms $\omega_{f}, \omega_{g, 1}, \omega_{g, 2}$ on $X$, the collection

$$
\omega_{f}, \omega_{g, 1}, \omega_{g, 2}, u \omega_{f}, u \omega_{g, 1}, u \omega_{g, 2}
$$

forms a basis for $H_{\mathrm{dR}}^{1}(X / \mathbf{Q})$. By finding the matrices of a few Hecke operators with respect to this basis, one can as in the case $N=37$ produce symplectic bases

$$
\omega_{f}, \eta_{f} \quad \text { and } \quad \omega_{g, 1}, \omega_{g, 2}, \eta_{g, 1}, \eta_{g, 2}
$$

for $H_{\mathrm{dR}}^{1}(X)[f]$ and $H_{\mathrm{dR}}^{1}(X)[g]$ respectively.

We can compute the Poincaré dual $\gamma_{f}$ and the iterated integrals

$$
\int_{\gamma_{f}}\left(\omega_{g, i} \cdot \omega_{g, j}-\alpha_{\omega_{g, i}, \omega_{g, j}}\right), \int_{\gamma_{f}}\left(\omega_{g, i} \cdot \eta_{g, j}-\alpha_{\omega_{g, i}, \eta_{g, j}}\right), \int_{\gamma_{f}}\left(\eta_{g, i} \cdot \omega_{g, j}-\alpha_{\eta_{g, i}, \omega_{g, j}}\right)
$$

in the same manner as in the case $N=37$ with one exception. One simply cannot find $\alpha_{\omega_{g, i}, \eta_{g, j}}$ by inspection. No linear combination of our chosen basis has the same principal part as $\omega_{g, i} F_{\eta_{g, j}}$, however some linear combination is cohomologous to such a form. The techniques from $\$ 4.5$ can be used to find such a form.

Each $T_{g, n}$ gives rise to an element of $\operatorname{End}\left(H_{\mathrm{dR}}^{1}(X)[g]\right) \otimes \mathbf{Q}$. The collection of elements arising from $T_{g, n}, n \geq 1$ generate a subspace of dimension 2 , generated by $T_{g, 1}$ and $T_{g, 2}$. Thus, we can effectively compute $P_{g, f, n}$ for all $n$ simply by computing $P_{g, f}$ and $P_{g, f, 2}$. The formula for $P_{g, f}$ is the one given in Remark 3.2.2, so we have

$$
\begin{aligned}
z_{g, f} & =\int_{\gamma_{f}}\left(\omega_{g, 1} \cdot \eta_{g, 1}-\eta_{g, 1} \cdot \omega_{g, 1}-2 \alpha_{\omega_{g, 1}, \eta_{g, 1}}+\omega_{g, 2} \cdot \eta_{g, 2}-\eta_{g, 2} \cdot \omega_{g, 2}-2 \alpha_{\omega_{g, 2}, \eta_{g, 2}}\right) \\
& =-2.0768300 \ldots+(2.7263648 \ldots) i
\end{aligned}
$$

The Hecke algebra $\mathbf{T}$ can be identified with $\mathbf{Q} \times \mathbf{Q}(\sqrt{2})$ via $T_{n} \otimes 1 \mapsto\left(a_{n}(f), a_{n}(g)\right)$. Under this identification, $T_{g, 1}$ corresponds to $e_{1}=(0,1)$, and an examination of the Fourier coefficients of $f$ and $g$ shows that $e_{1}$ does not lie in the image of $\mathbf{T}_{\mathbf{Z}}$, but $2 e_{1}$ does. So, we have

$$
P_{g, f}=W\left(2 z_{g, f}\right) \otimes \frac{1}{2}=\left(\frac{11}{49}:-\frac{363}{343}: 1\right) \otimes \frac{1}{2} \in E(\mathbf{Q}) \otimes \mathbf{Q} .
$$


Finding $P_{g, f, 2}$ is a little more involved, as we must compute the matrix of $T_{2}$ acting on $\omega_{g, 1}, \omega_{g, 2}, \eta_{g, 1}, \eta_{g, 2}$. Two methods for doing this were discussed in $₫ 4.2$ and 4.6. and either shows that $T_{2} \omega_{g, 1}=2 \omega_{g, 2}, T_{2} \omega_{g, 2}=\omega_{g, 1}, T_{2} \eta_{g, 1}=-\frac{97997}{132319} \omega_{g, 2}+$ $\eta_{g, 2}$ and $T_{2} \eta_{g, 2}=\frac{97997}{132319} \omega_{g, 1}+2 \eta_{g, 1}$. So the matrix $A_{2}$ is given by

$$
\left(\begin{array}{cccc}
0 & 2 & 0 & 0 \\
1 & 0 & 0 & 0 \\
0 & -\frac{97997}{132319} & 0 & 1 \\
\frac{97997}{132319} & 0 & 2 & 0
\end{array}\right) .
$$

Combining this with Lemma 3.2 .1 and remembering that $\alpha_{\omega_{g, i}, \omega_{g, j}}=0$ by Remark 2.2.6, we find that

$$
\begin{aligned}
z_{g, f, 2}= & -\frac{97997}{132319} \int_{\gamma_{f}}\left(\omega_{g, 1} \cdot \omega_{g, 2}-\omega_{g, 2} \cdot \omega_{g, 1}\right) \\
& +\int_{\gamma_{f}}\left(\omega_{g, 1} \cdot \eta_{g, 2}-\eta_{g, 2} \cdot \omega_{g, 1}-2 \alpha_{\omega_{g, 1}, \eta_{g, 2}}\right) \\
& +2 \int_{\gamma_{f}}\left(\omega_{g, 2} \cdot \eta_{g, 1}-\eta_{g, 1} \cdot \omega_{g, 2}-2 \alpha_{\omega_{g, 2}, \eta_{g, 1}}\right) \\
= & 2.4055874 \ldots-(1.0710898 \ldots) i .
\end{aligned}
$$

The cycle $T_{g, 2}$ corresponds to the element $e_{2}=(0, \sqrt{2})$ in $\mathbf{Q} \times \mathbf{Q}(\sqrt{2})$, which belongs to $\mathbf{T}_{\mathbf{Z}}$ by inspection of the Fourier coefficients of $f$ and $g$. By evaluating the Weierstrass uniformisation on $z_{g, f, s}$ we find:

$$
P_{g, f, 2}=W\left(z_{g, f, 2}\right) \otimes 1=(-1: 0: 1) \otimes 1 \in E(\mathbf{Q}) \otimes \mathbf{Q} .
$$

5.3. Table. In Table 1 we report some Chow-Heegner points that lie on elliptic curves over $\mathbf{Q}$ of rank 1 and conductor $<100$. The Chow-Heegner points in question were computed using a Sage $\left[\mathrm{S}^{+} 09\right]$ implementation of the algorithm described in the body of the paper; the whole table required several days of CPU time.

The format of the table is as follows. We list the strong Weil curve $E$ in each isogeny class of rank 1 . Let $N$ be the conductor of the curve $E$ in question, and $f \in S_{2}\left(\Gamma_{0}(N)\right)$ the newform corresponding to $E$. The first two columns of the table list the label for $E$ in Cremona's database and a choice of generator for $E(\mathbf{Q})$, using the canonical minimal Weierstrass equation for $E$ with invariants $a_{1}, a_{3} \in\{0,1\}$ and $a_{2} \in\{-1,0,1\}$.

The next column indicates a Galois orbit of normalized Hecke eigenforms $g \in$ $S_{2}\left(\Gamma_{0}(N)\right)$. The column gives the index of the orbit in question when the orbits are ordered lexicographically by the sequence of traces of $T_{1}$ and $T_{p}$ for all primes $p$, starting with index 0 . In particular, the trace of $T_{1}$ is equal to twice the dimension of $S_{2}(N)[g]$, so the components are sorted first by dimension, and rational newforms are always listed first. As a result, the $f$-isotypic component, which is omitted from the table, is typically the index 0 component. In parentheses we give the integer $N_{g} \mid N$ such that $g$ is associated to a newform of level $N_{g}$.

The fourth column gives an integer $n \geq 1$ corresponding to a Hecke operator $T_{n}$. The table includes $\left[K_{g}: \mathbf{Q}\right] \cdot \sigma\left(N / N_{g}\right)$ values of $n$ such that $T_{g} \cdot T_{n}$ form a Q-basis for the direct factor $K_{g}$ of $\mathbf{T}$. The fifth column lists $P_{g, f, n}$ as an element of $E(\mathbf{Q}) \otimes_{\mathbf{z}} \mathbf{Q}$, in terms of the generator $P$ in the second column. The sixth column gives the denominator $d_{g, n}$ defined above. 
TABLE 1. Chow-Heegner points on curves of rank 1 and conductor $<100$

\begin{tabular}{|c|c|c|c|c|c|}
\hline $\begin{array}{l}\text { Curve } \\
E=E_{f}\end{array}$ & $\begin{array}{l}\text { generator } \\
P \in E(\mathbf{Q})\end{array}$ & $g\left(N_{g}\right)$ & $n$ & $P_{g, f, n}$ & $d_{g, n}$ \\
\hline $37 \mathrm{a} 1$ & $(0,-1)$ & 1 (37) & 1 & $-6 P$ & 2 \\
\hline $43 \mathrm{a} 1$ & $(0,-1)$ & $1(43)$ & \begin{tabular}{|l|}
1 \\
2
\end{tabular} & $\begin{array}{l}4 P \\
2 P\end{array}$ & $\begin{array}{l}2 \\
1\end{array}$ \\
\hline $53 a 1$ & $(0,-1)$ & $1(53)$ & $\begin{array}{l}1 \\
2 \\
3 \\
\end{array}$ & $\begin{array}{r}-2 P \\
-8 P \\
4 P\end{array}$ & $\begin{array}{l}2 \\
2 \\
2\end{array}$ \\
\hline $57 \mathrm{a} 1$ & $(2,1)$ & $\begin{array}{ll}1 & (57) \\
2 & (57) \\
3 & (19)\end{array}$ & \begin{tabular}{l|l}
1 \\
1 \\
1 \\
3
\end{tabular} & $\begin{array}{r}\frac{4}{3} P \\
-\frac{16}{3} P \\
-4 P \\
-4 P\end{array}$ & $\begin{array}{r}12 \\
3 \\
2 \\
2\end{array}$ \\
\hline $58 \mathrm{a} 1$ & $(0,-1)$ & $\begin{array}{ll}1 & (58) \\
2 & (29)\end{array}$ & $\begin{array}{l}1 \\
1 \\
2 \\
3 \\
4\end{array}$ & $\begin{array}{r}4 P \\
0 \\
4 P \\
4 P \\
4 P\end{array}$ & $\begin{array}{l}4 \\
2 \\
2 \\
2 \\
2\end{array}$ \\
\hline 61a1 & $(1,-1)$ & $1(61)$ & \begin{tabular}{l|}
1 \\
2 \\
3
\end{tabular} & $\begin{array}{r}-2 P \\
4 P \\
-4 P\end{array}$ & $\begin{array}{l}2 \\
2 \\
1\end{array}$ \\
\hline 65a1 & $(-1,1)$ & $\begin{array}{l}1(65) \\
2(65)\end{array}$ & $\begin{array}{l}1 \\
2 \\
1 \\
2\end{array}$ & $\begin{array}{r}P \\
P \\
P \\
3 P\end{array}$ & $\begin{array}{l}2 \\
2 \\
2 \\
2\end{array}$ \\
\hline $77 \mathrm{a} 1$ & $(2,3)$ & $\begin{array}{ll}1 & (77) \\
2 & (77) \\
3 & (11) \\
4 & (77)\end{array}$ & \begin{tabular}{|l|l}
1 \\
1 \\
1 \\
7 \\
1 \\
2
\end{tabular} & $\begin{array}{r}\frac{12}{5} P \\
-\frac{4}{3} P \\
\frac{4}{3} P \\
\frac{44}{3} P \\
-\frac{12}{5} P \\
-4 P\end{array}$ & $\begin{array}{r}20 \\
6 \\
6 \\
6 \\
10 \\
2\end{array}$ \\
\hline \begin{tabular}{|l|}
$79 a 1$ \\
\end{tabular} & $(0,0)$ & $1(79)$ & \begin{tabular}{|l|}
1 \\
2 \\
3 \\
4 \\
5 \\
\end{tabular} & $\begin{array}{r}-4 P \\
-4 P \\
-4 P \\
0 \\
0\end{array}$ & $\begin{array}{l}2 \\
2 \\
2 \\
2 \\
2\end{array}$ \\
\hline $82 \mathrm{a} 1$ & $(0,0)$ & $\begin{array}{l}1(82) \\
2(41)\end{array}$ & $\begin{array}{l}1 \\
3 \\
1 \\
2 \\
3 \\
4 \\
5 \\
6\end{array}$ & $\begin{array}{r}0 \\
2 P \\
2 P \\
0 \\
2 P \\
0 \\
4 P \\
2 P\end{array}$ & $\begin{array}{l}4 \\
2 \\
2 \\
2 \\
2 \\
2 \\
2 \\
2\end{array}$ \\
\hline $83 a 1$ & $(0,0)$ & $1(83)$ & \begin{tabular}{|l|}
1 \\
2 \\
3 \\
4 \\
5 \\
7 \\
\end{tabular} & $\begin{array}{r}0 \\
2 P \\
4 P \\
-4 P \\
-4 P \\
0\end{array}$ & $\begin{array}{l}2 \\
2 \\
2 \\
2 \\
1 \\
2\end{array}$ \\
\hline
\end{tabular}

\begin{tabular}{|c|c|c|c|c|c|}
\hline $\begin{array}{l}\text { Curve } \\
E=E_{f}\end{array}$ & $\begin{array}{l}\text { generator } \\
P \in E(\mathbf{Q})\end{array}$ & $g\left(N_{g}\right)$ & $n$ & $P_{g, f, n}$ & $d_{g, n}$ \\
\hline $88 \mathrm{a} 1$ & $(2,-2)$ & $\begin{array}{l}1(88) \\
2(44) \\
3(11)\end{array}$ & $\begin{array}{l}1 \\
3 \\
1 \\
2 \\
1 \\
2 \\
4 \\
8\end{array}$ & $\begin{array}{r}0 \\
0 \\
0 \\
8 P \\
0 \\
8 P \\
16 P \\
16 P\end{array}$ & $\begin{array}{r}16 \\
16 \\
8 \\
2 \\
2 \\
2 \\
1 \\
1\end{array}$ \\
\hline $89 a 1$ & $(0,-1)$ & $\begin{array}{l}1(89) \\
2(89)\end{array}$ & $\begin{array}{l}1 \\
1 \\
2 \\
3 \\
4 \\
6\end{array}$ & $\begin{array}{r}\frac{8}{5} P \\
\frac{2}{5} P \\
\frac{22}{5} P \\
-\frac{16}{5} P \\
-\frac{2}{5} P \\
\frac{24}{5} P\end{array}$ & $\begin{array}{r}5 \\
10 \\
10 \\
10 \\
10 \\
10\end{array}$ \\
\hline 91a1 & $(0,0)$ & $\begin{array}{ll}1 & (91) \\
2 & (91) \\
3 & (91)\end{array}$ & $\begin{array}{l}1 \\
1 \\
2 \\
1 \\
2 \\
3\end{array}$ & $\begin{array}{r}2 P \\
2 P \\
-2 P \\
4 P \\
2 P \\
6 P\end{array}$ & $\begin{array}{l}4 \\
4 \\
2 \\
4 \\
2 \\
2\end{array}$ \\
\hline 91b1 & $(-1,3)$ & $\begin{array}{ll}0 & (91) \\
2 & (91) \\
3 & (91)\end{array}$ & $\begin{array}{l}1 \\
1 \\
2 \\
1 \\
2 \\
3\end{array}$ & $\begin{array}{l}0 \\
0 \\
0 \\
0 \\
0 \\
0\end{array}$ & $\begin{array}{l}4 \\
4 \\
2 \\
4 \\
2 \\
2\end{array}$ \\
\hline $92 \mathrm{~b} 1$ & $(1,1)$ & $\begin{array}{ll}1 & (92) \\
2 & (46) \\
3 & (23)\end{array}$ & $\begin{array}{l}1 \\
1 \\
2 \\
1 \\
2 \\
3 \\
4 \\
6 \\
8\end{array}$ & $\begin{array}{l}0 \\
0 \\
0 \\
0 \\
0 \\
0 \\
0 \\
0 \\
0\end{array}$ & $\begin{array}{r}2 \\
15 \\
5 \\
20 \\
5 \\
4 \\
6 \\
1 \\
5\end{array}$ \\
\hline 99a1 & $(2,0)$ & $\begin{array}{ll}1 & (99) \\
2 & (99) \\
3 & (99) \\
4 & (33) \\
5 & (11)\end{array}$ & $\begin{array}{l}1 \\
1 \\
1 \\
1 \\
3 \\
1 \\
3 \\
9\end{array}$ & $\begin{array}{r}-\frac{2}{3} P \\
0 \\
\frac{2}{3} P \\
\frac{2}{3} P \\
\frac{8}{3} P \\
-\frac{2}{3} P \\
-\frac{2}{3} P \\
-\frac{22}{3} P\end{array}$ & $\begin{array}{r}12 \\
12 \\
6 \\
12 \\
3 \\
6 \\
3 \\
3\end{array}$ \\
\hline
\end{tabular}


5.4. Further observations. We conclude with a few remarks.

5.4.1. Table 1 includes several examples where the submodule $\underline{P}_{\mathbf{T}[g], f} \subset \underline{P}_{g, f} \subset$ $E(\mathbf{Q}) \otimes_{\mathbf{Z}} \mathbf{Q}$ vanishes; namely, this occurs when $E$ has label 88a1 and $g$ has index 1 , for all $g$ when $E$ is labeled $91 \mathrm{~b} 1$ or 92b1, and when $E$ has label 99a1 and $g$ has index 2. This raises the question of whether such vanishing can be explained via Theorem 3.1.4.

At primes $p$ which exactly divide the level $N$, the local sign depends only on $E$ and can be computed easily from the Fourier expansion of $f$ as $\epsilon_{p}(g, g, f)=$ $-a_{p}(f)=1$.

Thus, for example, one can check that for the curve labeled 91b1 we have $\epsilon_{7}(g, g, f)=-a_{7}(f)=-1$ and $\epsilon_{13}(g, g, f)=-a_{13}(f)=-1$. Hence Theorem 3.1.4 does not apply; nevertheless, in this case we expect the special value $L(f \otimes$ $\left.\operatorname{Sym}^{2}(g), 2\right)$ not to vanish - a claim which should be in principle numerically verifiable by means of Dokchitser's $L$-function calculator (cf. $\left.\mathrm{S}^{+} 09\right]$ - and nontorsion points on $E(\mathbf{Q})$ to arise as the Chow-Heegner points associated to the Shimura curve of full level structure on the quaternion algebra of discriminant 91.

Similarly, one can explain the vanishing of $\underline{P}_{\mathbf{T}[g], f}$ on the elliptic curve $\mathbf{9 2 \mathbf { b } 1}$ because the prime $p=23$ exactly divides 92 and $\epsilon_{23}(g, g, f)=-a_{23}(f)=-1$.

As for the examples in Table 1 of torsion Chow-Heegner points at levels 88 and 99 , we have $\epsilon_{11}(g, g, f)=-a_{11}(f)=+1$ in both cases, but the local sign at 2 (respectively, at 3) is somewhat more dificult to compute. The reader interested in pursuing this computation will find it helpful to follow the recipe for that sign given by Prasad in [Pr90, which can be approached numerically thanks to the work of Loeffler and Weinstein LoWe.

In the tables of Stein (see Section 6.3) there is an example of a torsion ChowHeegner point corresponding to two newforms of level 158. Since this level is squarefree, the hypotheses of Theorem 3.1 .4 can be checked, and since the rank of the corresponding elliptic curve (labeled 158b1) is exactly 1, it would appear that $L\left(f \otimes \operatorname{Sym}^{2}(g), 2\right)=0$ in this example.

5.4.2. Examining Table 1, we see that in each example in which $f$ and $g$ are both newforms of level $N$ (such as when $E$ has label 37a1, or some of the examples when $E$ has label $\mathbf{5 7 a 1}, \mathbf{7 7 a 1}, \mathbf{9 9 a 1}$, etc.) the index $\left[E(\mathbf{Q}): \mathbf{Z} d_{g, 1} P_{f, g, 1}\right]$ is divisible by 4. See Section 6.5.3 for further discussion of this phenomenon.

5.4.3. Let $E=E_{f}$ be the elliptic curve with Cremona label 99a1. As $g$ runs through the Galois orbits of eigenforms of level 99, let the corresponding newforms of levels $N_{g} \mid 99$ be

$$
g_{99}^{(1)}, g_{99}^{(2)}, g_{99}^{(3)}, g_{33}, g_{11} \text {. }
$$

The newforms $g_{99}^{(1)}$ and $g_{99}^{(3)}$ are twists by the quadratic character of conductor 3 of, respectively, $g_{33}$ and $g_{11}$. Table 1 shows that in $E(\mathbf{Q}) \otimes \mathbf{Q}$ we have

$$
\begin{gathered}
P_{f, g_{99}^{(1)}, 1}=-P_{f, g_{33}, 1}=-\frac{2}{3} P, \\
P_{f, g_{99}^{(3)}, 1}=-P_{f, g_{11}, 1}=\frac{2}{3} P,
\end{gathered}
$$

where $P=(2: 0: 1) \in E(\mathbf{Q})$ is a generator. These observations are consistent with the expectation that not only the height (cf. Theorem 3.1.4) but even the exact index $\left[E(\mathbf{Q}): \mathbf{Z} d_{g, n} P_{g, f, n}\right]$ can be extracted from $L$-values of the form $L(f \otimes$ Sym $\left.^{2} g, 2\right)$ which are invariant under quadratic twists of $g$. 


\section{Appendix: Chow-Heegner points associated \\ TO PAIRS OF ELLIPTIC CURVES \\ by William Stein}

In this appendix, we consider a special case of Chow-Heegner points that has a simple concrete description due to S. Zhang. Given a pair $E, F$ of nonisogenous elliptic curves, and surjective morphisms $\varphi_{E}: X_{0}(N) \rightarrow E$ and $\varphi_{F}: X_{0}(N) \rightarrow F$ of curves over $\mathbf{Q}$, we associate a rational point $P \in E(\mathbf{Q})$. We describe a numerical approach to computing $P$, state some motivating results of Zhang et al. about the height of $P$, and present a table of data.

6.1. Introduction. Consider a pair $E, F$ of nonisogenous elliptic curves over $\mathbf{Q}$ and fix surjective morphisms from $X_{0}(N)$ to each curve. We do not assume that $N$ is the conductor of either $E$ or $F$, though $N$ is necessarily a multiple of the conductor.

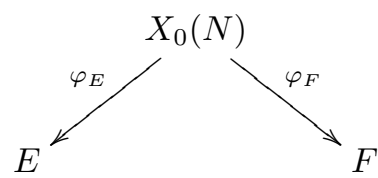

Let $\left(\varphi_{E}\right)_{*}: \operatorname{Div}\left(X_{0}(N)\right) \rightarrow \operatorname{Div}(E)$ and $\varphi_{F}^{*}: \operatorname{Div}(F) \rightarrow \operatorname{Div}\left(X_{0}(N)\right)$ be the pushforward and pullback maps on divisors on algebraic curves. Let $Q \in F(\mathbb{C})$ be any point, and let

$$
P_{\varphi_{E}, \varphi_{F}, Q}=\sum\left(\varphi_{E}\right)_{*} \varphi_{F}^{*}(Q) \in E(\mathbb{C}),
$$

where $\sum$ means the sum of the points in the divisor using the group law on $E$, i.e., given a divisor $D=\sum n_{i} P_{i} \in \operatorname{Div}(E)$, we have $\left(\sum D\right)-\infty \sim D-\operatorname{deg}(D) \infty$, which uniquely determines $\sum D$.

Proposition 6.1.1. The point $P_{\varphi_{E}, \varphi_{F}, Q}$ does not depend on the choice of $Q$.

Proof. The composition $\left(\varphi_{E}\right)_{*} \circ \varphi_{F}^{*}$ induces a homomorphism of elliptic curves

$$
\psi: \operatorname{Pic}^{0}(F)=\operatorname{Jac}(F) \rightarrow \operatorname{Jac}(E)=\operatorname{Pic}^{0}(E) .
$$

Our hypothesis that $E$ and $F$ are nonisogenous implies that $\psi=0$. We denote by $[D]$ the linear equivalence class of a divisor in the Picard group. If $Q^{\prime} \in F(\mathbb{C})$ is another point, then under the above composition of maps,

$$
\left[Q-Q^{\prime}\right] \mapsto\left[\left(\varphi_{E}\right)_{*} \varphi_{F}^{*}(Q)-\left(\varphi_{E}\right)_{*} \varphi_{F}^{*}\left(Q^{\prime}\right)\right]=\left[P_{Q}-P_{Q^{\prime}}\right]
$$

Thus the divisor $P_{Q}-P_{Q^{\prime}}$ is linearly equivalent to 0 . But $F$ has genus 1 , so there is no rational function on $F$ of degree 1 , hence $P_{Q}=P_{Q^{\prime}}$, as claimed.

We let $P_{\varphi_{E}, \varphi_{F}}=P_{\varphi_{E}, \varphi_{F}, Q} \in E(\mathbb{C})$, for any choice of $Q$.

Corollary 6.1.2. We have $P_{\varphi_{E}, \varphi_{F}} \in E(\mathbf{Q})$.

Proof. Taking $Q=\mathcal{O} \in F(\mathbf{Q})$, we see that the divisor $\left(\varphi_{E}\right)_{*} \circ \varphi_{F}^{*}(Q)$ is rational, so its sum is also rational.

In the rest of this appendix, we write $P_{E, F}=P_{\varphi_{E}, \varphi_{F}}$ when $E$ and $F$ are both optimal curves of the same conductor $N$, and $\varphi_{E}$ and $\varphi_{F}$ are as in Section 6.5.

In Section 6.2 we discuss an example in which $E$ and $F$ both have conductor 37. Section 6.3 is about a formula of Yuan-Zhang-Zhang for the height of $P_{E, F}$ in terms of the derivative of an $L$-function, in some cases. In Section 6.4 we discuss 
the connection between this appendix and the rest of the paper. The heart of the appendix is Section 6.5, which describes our numerical approach to approximating $P_{E, F}$. Finally, Section 6.5.2 presents a table of points $P_{E, F}$.

6.2. Example: $N=37$. The smallest conductor curve of rank 1 is the curve $E$ with Cremona label 37a (see [Cre]). The paper MSD74] discusses the modular curve $X_{0}(37)$ in detail. It gives the affine equation $y^{2}=-x^{6}-9 x^{4}-11 x^{2}+37$ for $X_{0}(37)$, and describes how $X_{0}(37)$ is equipped with three independent involutions $w, T$ and $S$. The quotient of $X_{0}(37)$ by $w$ is $E$, the quotient by $T$ is an elliptic curve $F$ with $F(\mathbf{Q}) \approx \mathbb{Z} / 3 \mathbb{Z}$ and Cremona label $37 \mathrm{~b}$, and the quotient by $S$ is the projective line $\mathbb{P}^{1}$.

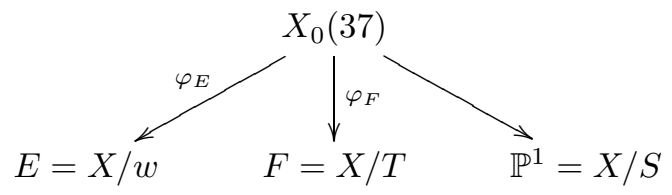

The maps $\varphi_{E}$ and $\varphi_{F}$ have degree 2 , by virtue of being induced by an involution. As explained in [MSD74, the fiber over $Q=0 \in F(\mathbf{Q})$ contains 2 points:

(1) the cusp $[\infty] \in X_{0}(37)(\mathbf{Q})$, and

(2) the noncuspidal affine rational point $(-1,-4)=T(\infty) \in X_{0}(37)(\mathbf{Q})$.

We have $\varphi_{E}([\infty])=0 \in E(\mathbf{Q})$, and [MSD74, Prop. 3, pg. 30] implies that

$$
\varphi_{F}((-1,-4))=(6,14)=-6(0,-1),
$$

where $(0,-1)$ generates $E(\mathbf{Q})$. We conclude that

$$
P_{E, F}=(6,14) \quad \text { and } \quad\left[E(\mathbf{Q}): \mathbb{Z} P_{E, F}\right]=6 .
$$

On MSD74, pg. 31], they remark: "It would be of utmost interest to link this index to something else in the theory."

This remark motivates our desire to compute more examples. Unfortunately, it is very difficult to generalize the above approach directly, since it involves computations with $X_{0}(37)$ and its quotients that rely on explicit defining equations. Just as there are multiple approaches to computing Heegner points, there are several approaches to computing $P_{E, F}$ :

- a Gross-Zagier style formula for the height of $P_{E, F}$ (see Section 6.3),

- explicit evaluation of iterated integrals (see Section 6.4), and

- numerical approximation of the fiber in the upper half-plane over a point on $F$ using a polynomial approximation to $\varphi_{F}$ (see Section 6.5).

This appendix is mainly about the last approach listed above.

6.3. The formula of Yuan-Zhang-Zhang. Consider a special case of the triple product $L$-function of [GK92,

$$
L(E, F, F, s)=L(E, s) \cdot L\left(E, \operatorname{Sym}^{2}(F), s\right),
$$

where $E$ and $F$ are elliptic curves of the same conductor $N$, and all $L$-functions are normalized so that $1 / 2$ is the center of the critical strip. The following theorem is proved in $\mathrm{YZZ}$ : 
Theorem 6.3.1 (Yuan-Zhang-Zhang). Assume that the local root number of $L(E, F, F, s)$ at every prime of bad reduction is +1 and that the root number at infinity is -1 . Then $\hat{h}\left(P_{E, F}\right)=(*) \cdot L^{\prime}\left(E, F, F, \frac{1}{2}\right)$, where $(*)$ is nonzero.

Remark 6.3.2. The above formula resembles the Gross-Zagier formula

$$
\hat{h}\left(P_{K}\right)=\left.(*) \cdot\left(L(E / \mathbf{Q}, s) \cdot L\left(E^{K} / \mathbf{Q}, s\right)\right)^{\prime}\right|_{s=\frac{1}{2}},
$$

where $K$ is a quadratic imaginary field satisfying certain hypotheses.

If one could evaluate $L^{\prime}\left(E, F, F, \frac{1}{2}\right)$, e.g., by applying the algorithm of Dok04, along with the factor $(*)$ in the theorem, this would yield an algorithm to compute $\pm P_{E, F}\left(\bmod E(\mathbf{Q})_{\text {tor }}\right)$ when the root number hypothesis is satisfied. Unfortunately, it appears that nobody has numerically evaluated the formula of Theorem 6.3.1 in any interesting cases.

When $E$ and $F$ have the same squarefree conductor $N$, GK92, $\S 1]$ implies that the local root number of $L(E, F, F, s)$ at $p$ is the same as the local root number of $E$ at $p$; computing the local root number when the level is not square free is more complicated.

Proposition 6.3.3. Assume that $E$ and $F$ have the same squarefree conductor $N$, that the local root numbers of $E$ at primes $p \mid N$ are all +1 (equivalently, that we have $\left.a_{p}(E)=-1\right)$ and that $r_{\mathrm{an}}(E / \mathbf{Q})=1$. Then $L\left(E, \operatorname{Sym}^{2} F, \frac{1}{2}\right) \neq 0$ if and only if $\hat{h}\left(P_{E, F}\right) \neq 0$.

Proof. By hypothesis, we have $L\left(E, \frac{1}{2}\right)=0$ and $L^{\prime}\left(E, \frac{1}{2}\right) \neq 0$. Theorem 6.3.1 and the factorization (6.3.1) imply that

$$
\hat{h}\left(P_{E, F}\right)=(*) \cdot L^{\prime}\left(E, \frac{1}{2}\right) \cdot L\left(E, \operatorname{Sym}^{2} F, \frac{1}{2}\right),
$$

from which the result follows.

Section 6.5.2 contains numerous examples in which $E$ has rank $1, F$ has rank 0, and yet $P_{E, F}$ is a torsion point. The first example is when $E$ is $91 \mathrm{~b}$ and $F$ is $91 \mathrm{a}$. Then $P_{E, F}=(1,0)$ is a torsion point (of order 3 ). In this case, we cannot apply Proposition 6.3.3 since $\epsilon_{7}=\epsilon_{13}=-1$ for $E$. Another example is when $E$ is $99 \mathrm{a}$ and $F$ is $99 \mathrm{c}$, where we have $P_{E, F}=0$, and $\epsilon_{3}=\epsilon_{11}=+1$, but Proposition 6.3.3 does not apply since the level is not square free. Fortunately, we found an example with squarefree level $158=2 \cdot 79$ : here $E$ is $158 \mathrm{~b}, F$ is $158 \mathrm{~d}$, we have $P_{E, F}=0$ and $\epsilon_{2}=\epsilon_{79}=+1$, so Proposition 6.3.3 implies that $L\left(E, \operatorname{Sym}^{2} F, \frac{1}{2}\right)=0$.

6.4. Iterated complex path integrals. The body of this paper contains a general approach using iterated path integrals to compute certain Chow-Heegner points, of which $P_{E, F}$ is a specific instance. Comparing our data (Section 6.5.2) with theirs, we find that if $E$ and $F$ are optimal elliptic curves over $\mathbf{Q}$ of the same conductor $N \leq 100$, if $e, f \in S_{2}\left(\Gamma_{0}(N)\right)$ are the corresponding newforms, and if $P_{f, e, 1} \in E(\mathbf{Q}) \otimes_{\mathbf{Q}} \mathbf{Q}$ is the associated Chow-Heegner point (as in the rest of this paper), then $2 P_{E, F}=P_{f, e, 1}$. This is a consequence of [Dau13], Proposition 3.3.3.

6.5. A numerical approach to computing $P_{E, F}$. The numerical approach to computing $P$ that we describe in this section uses relatively little abstract theory. It is inspired by work of Delaunay (see Del02]) on computing the fiber of the map $X_{0}(389) \rightarrow E$ over rational points on the rank 2 curve $E$ of conductor 389 . We make no guarantee about how many digits of our approximation to $P_{E, F}$ are 
correct, instead viewing this as an algorithm to produce something that is useful for experimental mathematics only.

Let $\mathfrak{h}$ be the upper half-plane, and let $Y_{0}(N)=\Gamma_{0}(N) \backslash \mathfrak{h} \subset X_{0}(N)$ be the affine modular curve. Let $E$ and $F$ be nonisogenous optimal elliptic curve quotients of $X_{0}(N)$, with modular parametrization maps $\varphi_{E}$ and $\varphi_{F}$, and assume both Manin constants are 1. Let $\Lambda_{E}$ and $\Lambda_{F}$ be the period lattices of $E$ and $F$, so $E \cong \mathbb{C} / \Lambda_{E}$ and $F \cong \mathbb{C} / \Lambda_{F}$. Viewed as a map $[\tau] \mapsto \mathbb{C} / \Lambda_{E}$, we have, using square brackets to denote equivalence classes, such that

$$
\varphi_{E}([\tau])=\left[\sum_{n=1}^{\infty} \frac{a_{n}}{n} e^{2 \pi i n \tau}\right],
$$

and similarly for $\varphi_{F}$, where $a_{n}=a_{n}(E)$ are the $L$-series coefficients of $E$ (see [Cr, §2.10], which uses the oppositive sign convention). For any positive integer $B$, define the polynomial

$$
\varphi_{E, B}=\sum_{n=1}^{B} \frac{a_{n}}{n} T^{n} \in \mathbf{Q}[T],
$$

and similarly for $\varphi_{F, B}$.

To approximate $P_{E, F}$, we proceed as follows. First we make some choices, and after making these choices we run the algorithm, which will either find a "probable" numerical approximation to $P_{E, F}$ or fail.

- $y \in \mathbb{R}_{>0}$ - minimum imaginary part of points in fiber,

- $d \in \mathbb{Z}_{>0}$ - degree of the first approximation to $\varphi_{F}$ in Step 1 below,

- $r \in \mathbb{R}_{\neq 0}$ - real number specified to $b$ bits of precision that defines $Q \in \mathbb{C} / \Lambda$,

- $b^{\prime}$ - bits of precision when dividing points into $\Gamma_{0}(N)$ orbits, and

- $n$ - number of trials before we give up and output FAIL.

We compute $P_{E, F, Q}$ using an approach that will always fail if $Q$ is a ramification point. Our algorithm will also fail if any points in the fiber over $Q$ are cusps. This is why we do not allow $r=0$. One can modify the algorithm to work when $Q$ is an unramified torsion point by using modular symbols and keeping track of images of cusps.

To increase our confidence that we have computed the right point $P_{E, F}$, we often carry out the complete computation with more than one choice of $r$.

(1) Low precision roots: Compute all complex double precision roots of the polynomial $\varphi_{F, d}-r$. One way to do this is to use "balanced $\mathrm{QR}$ reduction of the companion matrix", as implemented in GSL2 Record the roots that correspond to $\tau \in \mathfrak{h}$ with $\Im(\tau) \geq y$.

(2) High precision roots: Compute $B$ such that if $\Im(\tau) \geq y$, then

$$
\left|\sum_{n=B+1}^{\infty} \frac{a_{n}(F)}{n} \tau^{n}\right|<2^{-b},
$$

\footnotetext{
${ }^{2}$ GSL is the the GNU scientific library, which is part of Sage $\mathrm{S}^{+} 09$. Rough timings of GSL for this computation: it takes less than a half second for degree 500 , about 5 seconds for degree 1000 , about 45 seconds for degree 2000, and several minutes for degree 3000.
} 
where $b$ is the number of bits of precision of $r$. Summing the tail end of the series and using that $\left|a_{n}\right| \leq n$ (see $\mathrm{GJP}^{+} 09$, Lem. 2.9]), we find that

$$
B=\left\lceil\frac{\log \left(2^{-(b+1)} \cdot\left(1-e^{-2 \pi y_{1}}\right)\right)}{-2 \pi y}\right\rceil
$$

works. Next, compute the polynomial $\varphi_{F, B} \in \mathbf{Q}[T]$, and use Newton iteration to refine all roots saved in Step 1 to roots $\alpha$ of $f=\varphi_{F, B}-r \in \mathbb{R}[T]$ such that $|f(\alpha)|<2^{-b}$. Save those roots that correspond to $\tau \in \mathfrak{h}$ with $\Im(\tau) \geq y$.

(3) $\Gamma_{0}(N)$-orbits: Divide the $\tau$ 's from Step 2 into $\Gamma_{0}(N)$-equivalence classes, testing equivalence to the chosen bit precision $b^{\prime} \leq b$, as explained in Section 6.5.1. It is easy to efficiently compute the modular degree $m_{F}=$ $\operatorname{deg}\left(\varphi_{F}\right)$ (see Wat02]). If we find $m_{F}$ distinct $\Gamma_{0}(N)$ classes of points, we suspect that we have found the fiber over $[r]$, so we map each element of the fiber to $E$ using $\varphi_{E}$ and sum, then apply the elliptic exponential to obtain $P_{E, F}$ to some precision, then output this approximation and terminate. If we find more than $m_{F}$ distinct classes, there was an error in the choices of precision in our computation, so we output FAIL (and suggest either increasing $b$ or decreasing $b^{\prime}$ ).

(4) Try again: We did not find enough points in the fiber. Systematically replace $r$ by $r+m \Omega_{F}$, for $m=1,-1,2,-2, \ldots$, where $\Omega_{F}$ is the least real period of $F$, then try again going to Step 1 and including the new points found. If upon trying $n$ choices $r+m \Omega_{F}$ in a row we find no new points, we output FAIL and terminate the algorithm.

6.5.1. Determining $\Gamma_{0}(N)$ equivalency. The field of meromorphic functions invariant under $\Gamma_{0}(N)$ is generated by $j(z)$ and $j(N z)$, so if two points $z_{1}$ and $z_{2}$ in the upper half-plane are equivalent under $\Gamma_{0}(N)$, then $z_{1}$ and $z_{2}$ are equivalent under $\mathrm{SL}_{2}(\mathbb{Z})$ and $N z_{1}$ and $N z_{2}$ are also equivalent under $\mathrm{SL}_{2}(\mathbb{Z})$. Because of singularities in the affine curve defined by $j(z)$ and $j(N z)$, the converse is not true: for example, $z_{1}=(-2+i) / 5$ and $z_{2}=(2+i) / 5$ are equivalent under $\mathrm{SL}_{2}(\mathbb{Z})$ as are $5 z_{1}$ and $5 z_{2}$, but $z_{1}$ and $z_{2}$ are not equivalent under $\Gamma_{0}(5)$. This is why the algorithm we give below must take into account singularities.

Suppose we are given arbitrary $z_{1}$ and $z_{2}$ in the upper half-plane. We first find $g_{1}, g_{2} \in \mathrm{SL}_{2}(\mathbb{Z})$ such that $w_{i}=g_{i}\left(z_{i}\right)$ is the canonical representative for $z_{i}$ in the standard fundamental domain for $\mathrm{SL}_{2}(\mathbb{Z})$, as explained in $[\mathrm{Cr}$, §2.14] but using interval arithmetic to avoid rounding errors. If $w_{1} \neq w_{2}$, then $z_{1}$ and $z_{2}$ are not equivalent under $\mathrm{SL}_{2}(\mathbb{Z})$, so they cannot be equivalent under $\Gamma_{0}(N)$. Thus let $w=g_{1}\left(z_{1}\right)=g_{2}\left(z_{2}\right)$. The elements of $\mathrm{PSL}_{2}(\mathbb{Z})$ that send $z_{1}$ to $z_{2}$ are the finitely many elements $g_{2}^{-1} A g_{1}$, for $A \in \operatorname{Stab}(w)$, so we check whether any $g_{2}^{-1} A g_{1}$ is in $\Gamma_{0}(N)$. The only elements of the standard fundamental domain for $\mathrm{SL}_{2}(\mathbb{Z})$ with nontrivial stabilizers are $w=i$, with stabilizer generated by $S \in \mathrm{PSL}_{2}(\mathbb{Z})$ of order 2 , and $w=e^{2 \pi i / 3}$ with stabilizer generated by $S T$, where $T$ corresponds to $z \mapsto z+1$. 
6.5.2. Data. We implemented the above algorithm in Sage $\left[\mathrm{S}^{+} 09\right]^{3}$. The columns of Tables 2 5 are as follows. The columns labeled $E$ and $F$ contain Cremona labels for elliptic curves, and those labeled $r_{E}$ and $r_{F}$ contain the corresponding ranks. The column labeled $E(\mathbf{Q})$ gives a choice of generators $P_{1}, P_{2}, \ldots$ for the Mordell-Weil group, with $r_{E}$ points of infinite order listed first, then 0,1 or 2 torsion points listed with a subscript of their order. The column labeled $P_{E, F}$ contains a rational point close to the numerically computed Chow-Heegner point, represented in terms of the generators $P_{i}$ from the column labeled $E(\mathbf{Q})$, where $P_{1}$ is the first generator, $P_{2}$ the second, and so on. The columns labeled $m_{E}$ and $m_{F}$ give the modular degrees of $E$ and $F$. The column labeled $\epsilon$ 's contains the local root numbers of $L(E, s)$ at each bad prime. The notes column refers to the two notes below, which give information about the input parameters needed to compute $P_{E, F}$ :

(1) We used $y=10^{-5}$ and $d=1500$, which takes a few minutes.

(2) We used $y=\frac{1}{2} \cdot 10^{-5}$ and $d=3000$, which takes over an hour.

We believe that the values of $P_{E, F}$ are "likely" to be correct, but we emphasize again that they are not proven correct. In the table we give an exact point, but the algorithm computes a numerical approximation $\tilde{P}_{E, F}$ to $P_{E, F} \in E(\mathbf{Q})$. We find what we call $P_{E, F}$ in the table by running through several hundred low height points in $E(\mathbf{Q})$ and find the one closest to $\tilde{P}_{E, F}$; in all cases, the coordinates of the point we list are within $10^{-5}$ of the coordinates of $\tilde{P}_{E, F}$.

The table contains every pair $E, F$ of nonisogenous optimal elliptic curves of the same conductor $N \leq 184$ with $r_{E}=1$, and most (but not all) with $N \leq 250$. It also contains a few additional miscellaneous examples, e.g., with $r_{E}=0$ and some of larger conductor with $r_{F}=2$. Most rows took only a few seconds to compute, though ones with $m_{F}$ large in some cases took much longer; the total CPU time to compute the entire table was about 8 hours. Unless otherwise noted, we used $y=10^{-4}, d=500, b^{\prime}=20$, and $r=0.1$ with 53 bits of precision, as in Section 6.5. We also repeated all computations with $r=0.2$, and in every case got the same result.

6.5.3. Discussion. In Tables $2\left[5\right.$ we always have $2 \mid\left[E(\mathbf{Q})_{\text {tor }}: \mathbb{Z} P_{E, F}\right]$. In may be possible to prove this in some cases by using that when $r_{\text {an }}(E)=1$ then the sign in the functional equation for $L(E, s)$ is -1 , so at least one nontrivial AtkinLehner involution $w_{q}$ acts as +1 on $E$, which means that the map $X_{0}(N) \rightarrow E$ factors through $X_{0}(N) \rightarrow X_{0}(N) / w_{q}$. Also, there are four cases in which the index $\left[E(\mathbf{Q}) /\right.$ tor $\left.: \mathbb{Z} P_{E, F}\right]$ is divisible by a prime $\ell \geq 5$. They are $(106 \mathrm{~b}, 106 \mathrm{c}, \ell=11)$, $(118 \mathrm{a}, 118 \mathrm{~d}, \ell=7),(121 \mathrm{~b}, 121 \mathrm{~d}, \ell=7)$, and $(158 \mathrm{~b}, 158 \mathrm{c}, \ell=7)$. These prime divisors do not appear to have anything to do with the invariants of $E$ and $F$, individually.

\footnotetext{
${ }^{3}$ See http://trac.sagemath.org/sage_trac/ticket/11975.
} 
TABle 2. Chow-Heegner points associated to pairs of elliptic curves (part 1 of 4 )

\begin{tabular}{|c|c|c|c|c|c|c|c|c|c|}
\hline$E$ & $\epsilon_{p}$ 's & $r_{E}$ & $E(\mathbf{Q})$ & $m_{E}$ & $F$ & $r_{F}$ & $m_{F}$ & $P_{E, F}$ & Notes \\
\hline $37 \mathrm{a}$ & $\overline{+}$ & 1 & $(0,-1)$ & 2 & $37 \mathrm{~b}$ & 0 & 2 & $-6 P_{1}$ & \\
\hline $37 \mathrm{~b}$ & - & 0 & $(8,18)_{3}$ & 2 & $37 \mathrm{a}$ & 1 & 2 & $P_{1}$ & \\
\hline $57 \mathrm{a}$ & ++ & 1 & $(2,1)$ & 4 & $57 \mathrm{c}$ & 0 & 12 & $8 P_{1}$ & \\
\hline $57 \mathrm{a}$ & ++ & 1 & $(2,1)$ & 4 & $57 \mathrm{~b}$ & 0 & 3 & $-8 P_{1}$ & \\
\hline $57 \mathrm{~b}$ & -+ & 0 & $(7 / 4,-11 / 8)_{2},(1,-1)_{2}$ & 3 & $57 \mathrm{a}$ & 1 & 4 & 0 & \\
\hline $57 \mathrm{~b}$ & -+ & 0 & $(7 / 4,-11 / 8)_{2},(1,-1)_{2}$ & 3 & $57 \mathrm{c}$ & 0 & 12 & 0 & \\
\hline $57 \mathrm{c}$ & -+ & 0 & $(2,4)_{5}$ & 12 & $57 \mathrm{a}$ & 1 & 4 & $3 P_{1}$ & \\
\hline $57 \mathrm{c}$ & -+ & 0 & $(2,4)_{5}$ & 12 & $57 \mathrm{~b}$ & 0 & 3 & $P_{1}$ & \\
\hline $58 \mathrm{a}$ & ++ & 1 & $(0,-1)$ & 4 & $58 \mathrm{~b}$ & 0 & 4 & $8 P_{1}$ & \\
\hline $58 \mathrm{~b}$ & -+ & 0 & $(-1,2)_{5}$ & 4 & $58 \mathrm{a}$ & 1 & 4 & $3 P_{1}$ & \\
\hline $77 \mathrm{a}$ & ++ & 1 & $(2,3)$ & 4 & $77 \mathrm{~b}$ & 0 & 20 & $24 P_{1}$ & (1) \\
\hline $77 \mathrm{a}$ & ++ & 1 & $(2,3)$ & 4 & $77 \mathrm{c}$ & 0 & 6 & $-4 P_{1}$ & \\
\hline $89 a$ & + & 1 & $(0,-1)$ & 2 & $89 \mathrm{~b}$ & 0 & 5 & $4 P_{1}$ & \\
\hline $91 \mathrm{a}$ & ++ & 1 & $(0,0)$ & 4 & $91 \mathrm{~b}$ & 1 & 4 & $4 P_{1}$ & \\
\hline $91 \mathrm{~b}$ & -- & 1 & $(-1,3),(1,0)_{3}$ & 4 & $91 \mathrm{a}$ & 1 & 4 & $P_{2}$ & \\
\hline $92 \mathrm{~b}$ & -- & 1 & $(1,1)$ & 6 & $92 a$ & 0 & 2 & 0 & \\
\hline $99 a$ & ++ & 1 & $(2,0),(-1,0)_{2}$ & 4 & $99 \mathrm{~b}$ & 0 & 12 & $-4 P_{1}$ & \\
\hline $99 \mathrm{a}$ & ++ & 1 & $(2,0),(-1,0)_{2}$ & 4 & $99 \mathrm{c}$ & 0 & 12 & 0 & \\
\hline $99 \mathrm{a}$ & ++ & 1 & $(2,0),(-1,0)_{2}$ & 4 & $99 \mathrm{~d}$ & 0 & 6 & $2 P_{1}$ & \\
\hline $102 a$ & +++ & 1 & $(2,-4),(0,0)_{2}$ & 8 & $102 \mathrm{~b}$ & 0 & 16 & $-8 P_{1}$ & $(1)$ \\
\hline $102 a$ & +++ & 1 & $(2,-4),(0,0)_{2}$ & 8 & $102 \mathrm{c}$ & 0 & 24 & $32 P_{1}$ & \\
\hline $106 \mathrm{~b}$ & ++ & 1 & $(2,1)$ & 8 & $106 \mathrm{a}$ & 0 & 6 & $-4 P_{1}$ & \\
\hline $106 \mathrm{~b}$ & ++ & 1 & $(2,1)$ & 8 & $106 \mathrm{c}$ & 0 & 48 & $-88 P_{1}$ & \\
\hline $106 \mathrm{~b}$ & ++ & 1 & $(2,1)$ & 8 & $106 \mathrm{~d}$ & 0 & 10 & $12 P_{1}$ & \\
\hline $112 \mathrm{a}$ & ++ & 1 & $(0,-2),(-2,0)_{2}$ & 8 & $112 \mathrm{~b}$ & 0 & 4 & 0 & \\
\hline $112 \mathrm{a}$ & ++ & 1 & $(0,-2),(-2,0)_{2}$ & 8 & $112 \mathrm{c}$ & 0 & 8 & 0 & \\
\hline $118 \mathrm{a}$ & ++ & 1 & $(0,-1)$ & 4 & $118 \mathrm{~b}$ & 0 & 12 & $-8 P_{1}$ & $(1)$ \\
\hline $118 \mathrm{a}$ & ++ & 1 & $(0,-1)$ & 4 & $118 \mathrm{c}$ & 0 & 6 & $4 P_{1}$ & \\
\hline $118 \mathrm{a}$ & ++ & 1 & $(0,-1)$ & 4 & $118 \mathrm{~d}$ & 0 & 38 & $-28 P_{1}$ & \\
\hline $121 \mathrm{~b}$ & + & 1 & $(4,5)$ & 4 & $121 \mathrm{a}$ & 0 & 6 & $4 P_{1}$ & \\
\hline $121 \mathrm{~b}$ & + & 1 & $(4,5)$ & 4 & $121 \mathrm{c}$ & 0 & 6 & $4 P_{1}$ & \\
\hline $121 \mathrm{~b}$ & + & 1 & $(4,5)$ & 4 & $121 d$ & 0 & 24 & $-28 P_{1}$ & $(2)$ \\
\hline $123 \mathrm{a}$ & -- & 1 & $(-4,1),(-1,4)_{5}$ & 20 & 123b & 1 & 4 & 0 & \\
\hline $123 \mathrm{~b}$ & ++ & 1 & $(1,0)$ & 4 & $123 a$ & 1 & 20 & $4 P_{1}$ & \\
\hline $124 \mathrm{a}$ & -- & 1 & $(-2,1),(0,1)_{3}$ & 6 & 124b & 0 & 6 & 0 & \\
\hline $128 \mathrm{a}$ & + & 1 & $(0,1),(-1,0)_{2}$ & 4 & $128 \mathrm{~b}$ & 0 & 8 & 0 & \\
\hline $128 \mathrm{a}$ & + & 1 & $(0,1),(-1,0)_{2}$ & 4 & $128 \mathrm{c}$ & 0 & 4 & 0 & \\
\hline $128 \mathrm{a}$ & + & 1 & $(0,1),(-1,0)_{2}$ & 4 & $128 \mathrm{~d}$ & 0 & 8 & 0 & \\
\hline $129 \mathrm{a}$ & ++ & 1 & $(1,-5)$ & 8 & $129 \mathrm{~b}$ & 0 & 15 & $-8 P_{1}$ & \\
\hline $130 \mathrm{a}$ & +-- & 1 & $(-6,10),(-1,10)_{6}$ & 24 & 130b & 0 & 8 & 0 & \\
\hline $130 \mathrm{a}$ & +-- & 1 & $(-6,10),(-1,10)_{6}$ & 24 & $130 \mathrm{c}$ & 0 & 80 & 0 & \\
\hline $135 \mathrm{a}$ & ++ & 1 & $(4,-8)$ & 12 & $135 \mathrm{~b}$ & 0 & 36 & 0 & (1) \\
\hline $136 \mathrm{a}$ & -- & 1 & $(-2,2),(0,0)_{2}$ & 8 & $136 \mathrm{~b}$ & 0 & 8 & 0 & \\
\hline $138 \mathrm{a}$ & +++ & 1 & $(1,-2),(-2,1)_{2}$ & 8 & $138 \mathrm{~b}$ & 0 & 16 & $-16 P_{1}$ & (1) \\
\hline $138 \mathrm{a}$ & +++ & 1 & $(1,-2),(-2,1)_{2}$ & 8 & $138 \mathrm{c}$ & 0 & 8 & $-8 P_{1}$ & \\
\hline $141 \mathrm{a}$ & -- & 1 & $(-3,-5)$ & 28 & $141 \mathrm{~b}$ & 0 & 12 & 0 & \\
\hline $141 \mathrm{a}$ & -- & 1 & $(-3,-5)$ & 28 & 141c & 0 & 6 & 0 & \\
\hline $141 \mathrm{a}$ & -- & 1 & $(-3,-5)$ & 28 & $141 \mathrm{~d}$ & 1 & 4 & 0 & \\
\hline
\end{tabular}


TABle 3. Chow-Heegner points associated to pairs of elliptic curves (part 2 of 4 )

\begin{tabular}{|c|c|c|c|c|c|c|c|c|c|}
\hline$E$ & $\epsilon_{p}$ 's & $r_{E}$ & $E(\mathbf{Q})$ & $m_{E}$ & $F$ & $r_{F}$ & $m_{F}$ & $P_{E, F}$ & Notes \\
\hline $141 \mathrm{a}$ & - - & $\overline{1}$ & $(-3,-5)$ & 28 & $2141 \mathrm{e}$ & $\overline{0}$ & 12 & 0 & \\
\hline $141 d$ & ++ & 1 & $(0,-1)$ & 4 & $141 \mathrm{a}$ & 1 & 28 & $-12 P_{1}$ & \\
\hline $141 \mathrm{~d}$ & ++ & 1 & $(0,-1)$ & 4 & $141 \mathrm{~b}$ & 0 & 12 & $4 P_{1}$ & \\
\hline $141 d$ & ++ & 1 & $(0,-1)$ & 4 & $141 \mathrm{c}$ & 0 & 6 & $4 P_{1}$ & \\
\hline $141 d$ & ++ & 1 & $(0,-1)$ & 4 & $141 \mathrm{e}$ & 0 & 12 & $4 P_{1}$ & \\
\hline $142 \mathrm{a}$ & -- & 1 & $(1,1)$ & 36 & $142 \mathrm{~b}$ & 1 & 4 & 0 & \\
\hline $142 \mathrm{a}$ & -- & 1 & $(1,1)$ & 36 & $142 \mathrm{c}$ & 0 & 9 & 0 & \\
\hline $142 \mathrm{a}$ & -- & 1 & $(1,1)$ & 36 & $142 \mathrm{~d}$ & 0 & 4 & 0 & \\
\hline $142 \mathrm{a}$ & -- & 1 & $(1,1)$ & 36 & $142 \mathrm{e}$ & 0 & 324 & 0 & (2) \\
\hline $142 \mathrm{~b}$ & ++ & 1 & $(-1,0)$ & 4 & $142 \mathrm{a}$ & 1 & 36 & $4 P_{1}$ & $(1)$ \\
\hline $142 \mathrm{~b}$ & ++ & 1 & $(-1,0)$ & 4 & $142 \mathrm{c}$ & 0 & 9 & $-4 P_{1}$ & \\
\hline $142 \mathrm{~b}$ & ++ & 1 & $(-1,0)$ & 4 & $142 \mathrm{~d}$ & 0 & 4 & $4 P_{1}$ & \\
\hline $142 \mathrm{~b}$ & ++ & 1 & $(-1,0)$ & 4 & $142 \mathrm{e}$ & 0 & 324 & $8 P_{1}$ & $(2)$ \\
\hline $152 \mathrm{a}$ & ++ & 1 & $(-1,-2)$ & 8 & $152 \mathrm{~b}$ & 0 & 8 & 0 & \\
\hline $153 \mathrm{a}$ & ++ & 1 & $(0,1)$ & 8 & $153 \mathrm{~b}$ & 1 & 16 & $8 P_{1}$ & \\
\hline $153 \mathrm{a}$ & ++ & 1 & $(0,1)$ & 8 & $153 \mathrm{c}$ & 0 & 8 & $8 P_{1}$ & \\
\hline $153 \mathrm{a}$ & ++ & 1 & $(0,1)$ & 8 & $153 \mathrm{~d}$ & 0 & 24 & 0 & \\
\hline $153 \mathrm{~b}$ & -- & 1 & $(5,-14)$ & 16 & $153 \mathrm{a}$ & 1 & 8 & 0 & \\
\hline $153 \mathrm{~b}$ & -- & 1 & $(5,-14)$ & 16 & $153 \mathrm{~d}$ & 0 & 24 & 0 & \\
\hline $154 \mathrm{a}$ & +++ & 1 & $(5,3),(-6,3)_{2}$ & 24 & $154 \mathrm{~b}$ & 0 & 24 & $-24 P_{1}$ & \\
\hline $154 \mathrm{a}$ & +++ & 1 & $(5,3),(-6,3)_{2}$ & 24 & $154 \mathrm{c}$ & 0 & 16 & $16 P_{1}$ & \\
\hline $155 \mathrm{a}$ & -- & 1 & $(5 / 4,31 / 8),(0,2)_{5}$ & 20 & $155 \mathrm{~b}$ & 0 & 8 & 0 & \\
\hline $155 \mathrm{a}$ & -- & 1 & $(5 / 4,31 / 8),(0,2)_{5}$ & 20 & $155 \mathrm{c}$ & 1 & 4 & 0 & \\
\hline $155 \mathrm{c}$ & ++ & 1 & $(1,-1)$ & 4 & $155 \mathrm{a}$ & 1 & 20 & $-12 P_{1}$ & \\
\hline $155 \mathrm{c}$ & ++ & 1 & $(1,-1)$ & 4 & $155 \mathrm{~b}$ & 0 & 8 & $4 P_{1}$ & \\
\hline $156 \mathrm{a}$ & -+- & 1 & $(1,1),(2,0)_{2}$ & 12 & $156 \mathrm{~b}$ & 0 & 12 & 0 & $(1)$ \\
\hline $158 \mathrm{a}$ & -- & 1 & $(-1,-4)$ & 32 & $158 \mathrm{~b}$ & 1 & 8 & 0 & \\
\hline $158 \mathrm{a}$ & -- & 1 & $(-1,-4)$ & 32 & $158 \mathrm{c}$ & 0 & 48 & 0 & (1) \\
\hline $158 \mathrm{a}$ & -- & 1 & $(-1,-4)$ & 32 & $158 \mathrm{~d}$ & 0 & 40 & 0 & \\
\hline $158 \mathrm{a}$ & -- & 1 & $(-1,-4)$ & 32 & $158 \mathrm{e}$ & 0 & 6 & 0 & \\
\hline $158 \mathrm{~b}$ & ++ & 1 & $(0,-1)$ & 8 & $158 \mathrm{a}$ & 1 & 32 & $-8 P_{1}$ & \\
\hline $158 \mathrm{~b}$ & ++ & 1 & $(0,-1)$ & 8 & $158 \mathrm{c}$ & 0 & 48 & $-56 P_{1}$ & $(1)$ \\
\hline $158 \mathrm{~b}$ & ++ & 1 & $(0,-1)$ & 8 & $158 \mathrm{~d}$ & 0 & 40 & 0 & \\
\hline $158 \mathrm{~b}$ & ++ & 1 & $(0,-1)$ & 8 & $158 \mathrm{e}$ & 0 & 6 & $-8 P_{1}$ & \\
\hline $160 \mathrm{a}$ & ++ & 1 & $(2,-2),(1,0)_{2}$ & 8 & $160 \mathrm{~b}$ & 0 & 8 & 0 & \\
\hline $162 \mathrm{a}$ & ++ & 1 & $(-2,4),(1,1)_{3}$ & 12 & $162 \mathrm{~b}$ & 0 & 6 & 0 & \\
\hline $162 \mathrm{a}$ & ++ & 1 & $(-2,4),(1,1)_{3}$ & 12 & $162 \mathrm{c}$ & 0 & 6 & 0 & \\
\hline $162 \mathrm{a}$ & ++ & 1 & $(-2,4),(1,1)_{3}$ & 12 & $162 \mathrm{~d}$ & 0 & 12 & 0 & \\
\hline $170 \mathrm{a}$ & +-- & 1 & $(0,2),(1,-1)_{2}$ & 16 & $170 \mathrm{~d}$ & 0 & 12 & 0 & \\
\hline $170 \mathrm{a}$ & +-- & 1 & $(0,2),(1,-1)_{2}$ & 16 & $170 \mathrm{e}$ & 0 & 20 & 0 & \\
\hline $171 b$ & -- & 1 & $(2,-5)$ & 8 & $171 \mathrm{a}$ & 0 & 12 & 0 & \\
\hline $171 b$ & -- & 1 & $(2,-5)$ & 8 & $171 \mathrm{c}$ & 0 & 96 & 0 & (1) \\
\hline $171 b$ & -- & 1 & $(2,-5)$ & 8 & $171 \mathrm{~d}$ & 0 & 32 & 0 & \\
\hline $175 \mathrm{a}$ & -- & 1 & $(2,-3)$ & 8 & $175 \mathrm{~b}$ & 1 & 16 & 0 & (1) \\
\hline $175 \mathrm{a}$ & -- & 1 & $(2,-3)$ & 8 & $175 \mathrm{c}$ & 0 & 40 & 0 & (1) \\
\hline $175 b$ & ++ & 1 & $(-3,12)$ & 16 & $175 \mathrm{a}$ & 1 & 8 & $16 P_{1}$ & \\
\hline $175 b$ & ++ & 1 & $(-3,12)$ & 16 & $175 \mathrm{c}$ & 0 & 40 & $16 P_{1}$ & $(1)$ \\
\hline $176 \mathrm{c}$ & -- & 1 & $(1,-2)$ & 8 & $176 \mathrm{~b}$ & 0 & 8 & 0 & (1) \\
\hline
\end{tabular}


TABle 4. Chow-Heegner points associated to pairs of elliptic curves (part 3 of 4 )

\begin{tabular}{|c|c|c|c|c|c|c|c|c|c|}
\hline$E$ & $\epsilon_{p}^{\prime}$ 's & $r_{E}$ & $E(\mathbf{Q})$ & $m_{E}$ & $F$ & $r_{F}$ & $m_{F}$ & $P_{E, F}$ & Notes \\
\hline $176 \mathrm{c}$ & -- & $\overline{1}$ & $(1,-2)$ & 8 & $176 \mathrm{a}$ & $\overline{0}$ & 16 & 0 & \\
\hline $176 \mathrm{c}$ & -- & 1 & $(1,-2)$ & 8 & $176 \mathrm{~b}$ & 0 & 8 & 0 & (1) \\
\hline $184 a$ & -- & 1 & $(0,1)$ & 8 & $184 \mathrm{c}$ & 0 & 12 & 0 & \\
\hline $184 a$ & -- & 1 & $(0,1)$ & 8 & $184 d$ & 0 & 24 & 0 & \\
\hline $184 \mathrm{~b}$ & ++ & 1 & $(2,-1)$ & 8 & $184 a$ & 1 & 8 & 0 & \\
\hline $184 \mathrm{~b}$ & ++ & 1 & $(2,-1)$ & 8 & $184 \mathrm{c}$ & 0 & 12 & 0 & \\
\hline $184 \mathrm{~b}$ & ++ & 1 & $(2,-1)$ & 8 & $184 \mathrm{~d}$ & 0 & 24 & 0 & \\
\hline $185 \mathrm{a}$ & ++ & 1 & $(4,-13)$ & 48 & $185 \mathrm{~b}$ & 1 & 8 & $8 P_{1}$ & \\
\hline $185 \mathrm{a}$ & ++ & 1 & $(4,-13)$ & 48 & $185 \mathrm{c}$ & 1 & 6 & $24 P_{1}$ & \\
\hline $185 \mathrm{~b}$ & -- & 1 & $(0,2)$ & 8 & $185 \mathrm{c}$ & 1 & 6 & 0 & \\
\hline $185 \mathrm{c}$ & ++ & 1 & $(-5 / 4,3 / 8),(-1,0)_{2}$ & 6 & $185 \mathrm{~b}$ & 1 & 8 & $2 P_{1}$ & \\
\hline $189 \mathrm{a}$ & ++ & 1 & $(-1,-2)$ & 12 & $189 \mathrm{~b}$ & 1 & 12 & $-12 P_{1}$ & \\
\hline $189 \mathrm{a}$ & ++ & 1 & $(-1,-2)$ & 12 & $189 \mathrm{c}$ & 0 & 12 & $12 P_{1}$ & \\
\hline $189 \mathrm{~b}$ & -- & 1 & $(-3,9),(3,0)_{3}$ & 12 & $189 \mathrm{a}$ & 1 & 12 & 0 & \\
\hline $189 \mathrm{~b}$ & -- & 1 & $(-3,9),(3,0)_{3}$ & 12 & $189 \mathrm{c}$ & 0 & 12 & 0 & \\
\hline $190 \mathrm{a}$ & -+- & 1 & $(13,-47)$ & 88 & $190 \mathrm{~b}$ & 1 & 8 & 0 & \\
\hline $190 \mathrm{a}$ & -+- & 1 & $(13,-47)$ & 88 & $190 \mathrm{c}$ & 0 & 24 & 0 & (1) \\
\hline $190 \mathrm{~b}$ & +++ & 1 & $(1,2)$ & 8 & $190 \mathrm{c}$ & 0 & 24 & $16 P_{1}$ & (1) \\
\hline $192 \mathrm{a}$ & ++ & 1 & $(3,2),(-1,0)_{2}$ & 8 & $192 b$ & 0 & 8 & 0 & \\
\hline $192 \mathrm{a}$ & ++ & 1 & $(3,2),(-1,0)_{2}$ & 8 & $192 \mathrm{c}$ & 0 & 8 & 0 & \\
\hline $192 \mathrm{a}$ & ++ & 1 & $(3,2),(-1,0)_{2}$ & 8 & $192 \mathrm{~d}$ & 0 & 8 & 0 & \\
\hline $196 \mathrm{a}$ & -- & 1 & $(0,-1)$ & 6 & $196 \mathrm{~b}$ & 0 & 42 & 0 & $(1)$ \\
\hline $198 \mathrm{a}$ & +-- & 1 & $(-1,-4),(-4,2)_{2}$ & 32 & $198 \mathrm{~b}$ & 0 & 32 & 0 & (1) \\
\hline $198 \mathrm{a}$ & +-- & 1 & $(-1,-4),(-4,2)_{2}$ & 32 & $198 \mathrm{c}$ & 0 & 32 & 0 & \\
\hline $198 \mathrm{a}$ & +-- & 1 & $(-1,-4),(-4,2)_{2}$ & 32 & $198 \mathrm{~d}$ & 0 & 32 & 0 & (1) \\
\hline $198 \mathrm{a}$ & +-- & 1 & $(-1,-4),(-4,2)_{2}$ & 32 & $198 \mathrm{e}$ & 0 & 160 & 0 & $(1)$ \\
\hline $200 \mathrm{~b}$ & -- & 1 & $(-1,1),(-2,0)_{2}$ & 8 & $200 \mathrm{c}$ & 0 & 24 & 0 & \\
\hline $200 \mathrm{~b}$ & -- & 1 & $(-1,1),(-2,0)_{2}$ & 8 & $200 \mathrm{~d}$ & 0 & 40 & 0 & (1) \\
\hline $200 \mathrm{~b}$ & -- & 1 & $(-1,1),(-2,0)_{2}$ & 8 & $200 \mathrm{e}$ & 0 & 24 & 0 & \\
\hline $201 \mathrm{a}$ & ++ & 1 & $(1,-2)$ & 12 & $201 \mathrm{~b}$ & 1 & 12 & $4 P_{1}$ & \\
\hline $201 b$ & -- & 1 & $(-1,2)$ & 12 & $201 \mathrm{a}$ & 1 & 12 & 0 & \\
\hline $201 \mathrm{c}$ & ++ & 1 & $(16,-7)$ & 60 & $201 \mathrm{a}$ & 1 & 12 & $-24 P_{1}$ & \\
\hline $201 \mathrm{c}$ & ++ & 1 & $(16,-7)$ & 60 & $201 \mathrm{~b}$ & 1 & 12 & $8 P_{1}$ & \\
\hline $203 \mathrm{~b}$ & -- & 1 & $(2,-5)$ & 8 & $203 a$ & 0 & 48 & 0 & \\
\hline $203 \mathrm{~b}$ & -- & 1 & $(2,-5)$ & 8 & $203 \mathrm{c}$ & 0 & 12 & 0 & \\
\hline $205 a$ & -- & 1 & $(-1,8),(2,1)_{4}$ & 12 & $205 \mathrm{~b}$ & 0 & 16 & 0 & \\
\hline $205 a$ & -- & 1 & $(-1,8),(2,1)_{4}$ & 12 & $205 \mathrm{c}$ & 0 & 8 & 0 & \\
\hline $208 \mathrm{a}$ & -- & 1 & $(4,-8)$ & 16 & $208 \mathrm{c}$ & 0 & 12 & 0 & \\
\hline $208 \mathrm{a}$ & -- & 1 & $(4,-8)$ & 16 & $208 \mathrm{~d}$ & 0 & 48 & 0 & (1) \\
\hline $208 b$ & ++ & 1 & $(4,4)$ & 16 & $208 \mathrm{a}$ & 1 & 16 & 0 & (1) \\
\hline $208 \mathrm{~b}$ & ++ & 1 & $(4,4)$ & 16 & $208 \mathrm{c}$ & 0 & 12 & 0 & \\
\hline $208 b$ & ++ & 1 & $(4,4)$ & 16 & $208 \mathrm{~d}$ & 0 & 48 & 0 & (1) \\
\hline $212 \mathrm{a}$ & -- & 1 & $(2,2)$ & 12 & $212 b$ & 0 & 21 & 0 & \\
\hline $214 a$ & -- & 1 & $(0,-4)$ & 28 & $214 \mathrm{~b}$ & 1 & 12 & 0 & (1) \\
\hline $214 a$ & -- & 1 & $(0,-4)$ & 28 & $214 d$ & 0 & 12 & 0 & \\
\hline $214 b$ & ++ & 1 & $(0,0)$ & 12 & $214 a$ & 1 & 28 & $-8 P_{1}$ & (1) \\
\hline $214 b$ & ++ & 1 & $(0,0)$ & 12 & $214 d$ & 0 & 12 & $-4 P_{1}$ & \\
\hline
\end{tabular}


TABle 5. Chow-Heegner points associated to pairs of elliptic curves (part 4 of 4 )

\begin{tabular}{|c|c|c|c|c|c|c|c|c|c|}
\hline$E$ & $\epsilon_{p}$ 's & $r_{E}$ & $E(\mathbf{Q})$ & $m_{E}$ & $F$ & $r_{F}$ & $m_{F}$ & $P_{E, F}$ & Notes \\
\hline $214 \mathrm{c}$ & ++ & 1 & $(11,10)$ & 60 & $214 \mathrm{a}$ & 1 & 28 & $-4 P_{1}$ & (1) \\
\hline $214 \mathrm{c}$ & ++ & 1 & $(11,10)$ & 60 & $214 \mathrm{~d}$ & 0 & 12 & $16 P_{1}$ & \\
\hline $214 \mathrm{c}$ & ++ & 1 & $(11,10)$ & 60 & $214 \mathrm{~b}$ & 1 & 12 & $12 P_{1}$ & (1) \\
\hline $216 \mathrm{a}$ & ++ & 1 & $(-2,-6)$ & 24 & $216 \mathrm{~b}$ & 0 & 24 & 0 & \\
\hline $219 a$ & ++ & 1 & $(2,-1)$ & 12 & $219 \mathrm{c}$ & 1 & 60 & $-12 P_{1}$ & (1) \\
\hline $219 a$ & ++ & 1 & $(2,-1)$ & 12 & $219 b$ & 1 & 12 & $-4 P_{1}$ & \\
\hline $216 \mathrm{a}$ & ++ & 1 & $(-2,-6)$ & 24 & $216 \mathrm{~d}$ & 0 & 72 & 0 & \\
\hline $219 \mathrm{~b}$ & -- & 1 & $(-3 / 4,-1 / 8),(0,1)_{3}$ & 12 & $219 \mathrm{a}$ & 1 & 12 & 0 & \\
\hline $219 \mathrm{~b}$ & -- & 1 & $(-3 / 4,-1 / 8),(0,1)_{3}$ & 12 & $219 \mathrm{c}$ & 1 & 60 & 0 & $(1)$ \\
\hline $219 \mathrm{c}$ & ++ & 1 & $(-6,7),(10,-5)_{2}$ & 60 & $219 a$ & 1 & 12 & $-12 P_{1}$ & \\
\hline $219 \mathrm{c}$ & ++ & 1 & $(-6,7),(10,-5)_{2}$ & 60 & $219 \mathrm{~b}$ & 1 & 12 & $4 P_{1}$ & \\
\hline $220 \mathrm{a}$ & --+ & 1 & $(-7,11),(15,55)_{6}$ & 36 & $220 \mathrm{~b}$ & 0 & 12 & 0 & \\
\hline $224 \mathrm{a}$ & ++ & 1 & $(1,2),(0,0)_{2}$ & 8 & $224 \mathrm{~b}$ & 0 & 8 & 0 & \\
\hline $225 \mathrm{a}$ & ++ & 1 & $(1,1)$ & 8 & $225 \mathrm{~b}$ & 0 & 40 & 0 & $(1)$ \\
\hline $225 \mathrm{e}$ & -- & 1 & $(-5,22)$ & 48 & $225 \mathrm{a}$ & 1 & 8 & 0 & (1) \\
\hline $225 \mathrm{e}$ & -- & 1 & $(-5,22)$ & 48 & $225 \mathrm{~b}$ & 0 & 40 & 0 & $(1)$ \\
\hline $228 \mathrm{~b}$ & -+- & 1 & $(3,6)$ & 24 & $228 \mathrm{a}$ & 0 & 18 & 0 & \\
\hline $232 \mathrm{a}$ & ++ & 1 & $(2,-4)$ & 16 & $232 \mathrm{~b}$ & 0 & 16 & 0 & \\
\hline $234 \mathrm{c}$ & +++ & 1 & $(1,-2),(-2,1)_{2}$ & 16 & $234 \mathrm{~b}$ & 0 & 48 & 0 & $(1)$ \\
\hline $234 \mathrm{c}$ & +++ & 1 & $(1,-2),(-2,1)_{2}$ & 16 & $234 \mathrm{e}$ & 0 & 20 & 0 & (1) \\
\hline $235 \mathrm{a}$ & -- & 1 & $(-2,3)$ & 12 & $235 \mathrm{c}$ & 0 & 18 & 0 & $(1)$ \\
\hline $236 \mathrm{a}$ & -- & 1 & $(1,-1)$ & 6 & $236 \mathrm{~b}$ & 0 & 14 & 0 & \\
\hline $238 \mathrm{a}$ & --+ & 1 & $(24,100),(-8,4)_{2}$ & 112 & $238 \mathrm{~b}$ & 1 & 8 & 0 & (1) \\
\hline $238 \mathrm{a}$ & --+ & 1 & $(24,100),(-8,4)_{2}$ & 112 & $238 \mathrm{c}$ & 0 & 16 & 0 & (1) \\
\hline $238 \mathrm{a}$ & --+ & 1 & $(24,100),(-8,4)_{2}$ & 112 & $238 \mathrm{~d}$ & 0 & 16 & 0 & (1) \\
\hline $238 b$ & +++ & 1 & $(1,1),(0,0)_{2}$ & 8 & $238 \mathrm{a}$ & 1 & 112 & $12 P_{1}$ & (1) \\
\hline $238 \mathrm{~b}$ & +++ & 1 & $(1,1),(0,0)_{2}$ & 8 & $238 \mathrm{c}$ & 0 & 16 & $-4 P_{1}$ & (1) \\
\hline $238 \mathrm{~b}$ & +++ & 1 & $(1,1),(0,0)_{2}$ & 8 & $238 \mathrm{~d}$ & 0 & 16 & $4 P_{1}$ & $(1)$ \\
\hline $240 \mathrm{c}$ & +++ & 1 & $(1,2),(0,0)_{2}$ & 16 & $240 \mathrm{a}$ & 0 & 16 & 0 & \\
\hline $240 \mathrm{c}$ & +++ & 1 & $(1,2),(0,0)_{2}$ & 16 & $240 \mathrm{~d}$ & 0 & 16 & 0 & (1) \\
\hline $243 a$ & + & 1 & $(1,0)$ & 6 & $243 \mathrm{~b}$ & 0 & 9 & 0 & (1) \\
\hline $245 a$ & -- & 1 & $(7,17)$ & 48 & $245 \mathrm{c}$ & 1 & 32 & 0 & \\
\hline $246 \mathrm{~d}$ & +++ & 1 & $(3,-6),(4,-2)_{2}$ & 48 & $246 \mathrm{a}$ & 0 & 84 & $24 P_{1}$ & (1) \\
\hline $446 \mathrm{a}$ & ++ & 1 & $(4,-6)$ & 24 & $446 \mathrm{~d}$ & 2 & 88 & 0 & $(2)$ \\
\hline $446 \mathrm{~b}$ & -- & 1 & $(5,-10)$ & 56 & $446 \mathrm{~d}$ & 2 & 88 & 0 & $(2)$ \\
\hline $446 \mathrm{~d}$ & +- & 2 & - & 88 & $446 \mathrm{a}$ & 1 & 12 & 0 & $(1)$ \\
\hline $446 \mathrm{~d}$ & +- & 2 & - & 88 & $446 \mathrm{~b}$ & 1 & 56 & 0 & (1) \\
\hline $681 \mathrm{a}$ & ++ & 1 & $(4,4)$ & 32 & $681 \mathrm{c}$ & 2 & 96 & $-24 P_{1}$ & $(2)$ \\
\hline
\end{tabular}




\section{ACKNOWLEDGEMENTS}

The authors would like to thank Ralph Greenberg, Ken Ribet, Barry Mazur, Karl Rubin, Shouwu Zhang, and Jon Bober for helpful conversations related to this paper, and they especially thank Xinyi Yuan for introducing us to this topic, Henri Darmon for encouraging us to flesh out the details and write it up for publication, and Victor Rotger for clarifying some issues in Section 6.3. We would also like to thank John Cremona for refereeing the corresponding Sage $\underline{\mathrm{S}^{+} 09}$ code and Cremona, Bas Edixhoven and Samit Dasgupta for contributions to Section 6.5.1.

The authors warmly thank the organizers of the 2011 Arizona Winter School for inviting the first and fourth author to deliver a series of lectures at the meeting, for creating such an inspiring atmosphere and giving the four of us the opportunity to work together.

\section{REFERENCES}

[ARS06] A. Agashe, K. Ribet, and W. A. Stein, The Manin constant, Pure Appl. Math. Q. 2 (2006), no. 2, 617-636, DOI 10.4310/PAMQ.2006.v2.n2.a11. MR2251484|(2007c:11076)

[BD1] M. Bertolini and H. Darmon, Kolyvagin's descent and Mordell-Weil groups over ring class fields, J. Reine Angew. Math. 412 (1990), 63-74, DOI 10.1515/crll.1990.412.63. MR $1079001(91 \mathrm{j}: 11048)$

[BD2] M. Bertolini and H. Darmon, Heegner points, p-adic L-functions, and the Cerednik-Drinfeld uniformization, Invent. Math. 131 (1998), no. 3, 453-491, DOI 10.1007/s002220050211. MR:1614543 (99f:11080)

[BDP1] M. Bertolini, H. Darmon, and K. Prasanna, Generalized Heegner cycles and p-adic Rankin L-series, Duke Math. J. 162 (2013), no. 6, 1033-1148, DOI 10.1215/001270942142056. With an appendix by Brian Conrad. MR.3053566

[BDP2] M. Bertolini, H. Darmon, and K. Prasanna, Chow-Heegner points on CM elliptic curves and values of p-adic L-series, Int. Math. Res. Not. IMRN 3 (2014), 745-793. MR.3163566

[Bi] B. Birch, Heegner points: the beginnings, Heegner points and Rankin L-series, Math. Sci. Res. Inst. Publ., vol. 49, Cambridge Univ. Press, Cambridge, 2004, pp. 1-10, DOI 10.1017/CBO9780511756375.002. MR2083207 (2005d:11083)

[BG] B. Birch and B. Gross, Correspondence, Heegner points and Rankin L-series, Math. Sci. Res. Inst. Publ., vol. 49, Cambridge Univ. Press, Cambridge, 2004, pp. 11-23, DOI 10.1017/CBO9780511756375.003. MR2083208 (2005g:11120)

[BO] K. Bringmann, K. Ono, Coefficients of harmonic weak Maass forms, Proceedings of the 2008 University of Florida conference on partitions, $q$-series, and modular forms.

[BrPh] N. Brisebarre and G. Philibert, Effective lower and upper bounds for the Fourier coefficients of powers of the modular invariant j, J. Ramanujan Math. Soc. 20 (2005), no. 4, 255-282. MR2193216 (2006k:11074)

[Ch] K. T. Chen, Iterated path integrals, Bull. Amer. Math. Soc. 83 (1977), no. 5, 831-879. MR0454968 (56 \#13210)

[Cr] J. Cremona, Chapter 2: Modular Symbol Algorithms, in Algorithms for Modular Elliptic curves. http://www.warwick.ac.uk/ masgaj/book/fulltext/index.html.

[Cre] J.E. Cremona, Elliptic Curves Data, http://www.warwick.ac.uk/ masgaj/ftp/data/.

[CWZ] J. A. Csirik, J. L. Wetherell, M. E. Zieve, On the genera of $X_{0}(N)$, preprint arXiv: math/0006096.

[D1] H. Darmon, Integration on $\mathcal{H}_{p} \times \mathcal{H}$ and arithmetic applications, Ann. of Math. (2) 154 (2001), no. 3, 589-639, DOI 10.2307/3062142. MR.1884617 (2003j:11067)

[D2] H. Darmon, Rational Points on Modular Elliptic Curves, CBMS Regional Conference Series in Mathematics, vol. 101, Published for the Conference Board of the Mathematical Sciences, Washington, DC, 2004. MR2020572 (2004k:11103)

[D3] H. Darmon, Cycles on modular varieties and rational points on elliptic curves, in Explicit Methods in Number Theory, Oberwolfach reports 6:3 (2009), 1843-1920. 
[DL] H. Darmon and A. Logan, Periods of Hilbert modular forms and rational points on elliptic curves, Int. Math. Res. Not. 40 (2003), 2153-2180, DOI 10.1155/S1073792803131108. MR1997296 (2005f:11110)

[DP] H. Darmon and R. Pollack, Efficient calculation of Stark-Heegner points via overconvergent modular symbols, Israel J. Math. 153 (2006), 319-354, DOI 10.1007/BF02771789. MR.2254648 (2007k:11077)

[DLR] H. Darmon, A. Lauder, V. Rotger, Stark points and p-adic iterated integrals attached to modular forms of weight one, submitted.

[DRS] H. Darmon, V. Rotger, and I. Sols, Iterated integrals, diagonal cycles and rational points on elliptic curves (English, with English and French summaries), Publications mathématiques de Besançon. Algèbre et théorie des nombres, 2012/2, Publ. Math. Besançon Algèbre Théorie Nr., vol. 2012/, Presses Univ. Franche-Comté, Besançon, 2012, pp. 19-46. MR3074917

[DR] H. Darmon, V. Rotger, Diagonal cycles and Euler systems I: A p-adic Gross-Zagier formula, Annales Sc. École Normal Supérieure, 47 (2014), no. 4, 779-832. MR 3250064

[Dau13] M. Daub, Complex and p-adic computations of Chow-Heegner points, Ph.D. Thesis, University of California, Berkeley.

[Del02] C. Delaunay, Formes modulaires et invariants de courbes elliptiques définies sur $\mathbf{Q}$, Thèse de Doctorat, Université Bordeaux I, available at http://math.univ-lyon1.fr/ delaunay/.

[DS] F. Diamond and J. Shurman, A First Course in Modular Forms, Graduate Texts in Mathematics, vol. 228, Springer-Verlag, New York, 2005. MR2112196 (2006f:11045)

[Dok04] T. Dokchitser, Computing special values of motivic L-functions, Experiment. Math. 13 (2004), no. 2, 137-149. MR2068888 (2005f:11128)

[E] M. Eichler, Quaternäre quadratische Formen und die Riemannsche Vermutung für die Kongruenzzetafunktion (German), Arch. Math. 5 (1954), 355-366. MR0063406 $(16,116 \mathrm{~d})$

[G] J. Gonzàlez Rovira, Equations of hyperelliptic modular curves (English, with French summary), Ann. Inst. Fourier (Grenoble) 41 (1991), no. 4, 779-795. MR.1150566 (93g:11064)

[Gr] M. Greenberg, Heegner Points and Rigid Analytic Modular Forms, ProQuest LLC, Ann Arbor, MI, 2006. Thesis (Ph.D.)-McGill University (Canada). MR2710023

[GJP +09$]$ G. Grigorov, A. Jorza, S. Patrikis, W. A. Stein, and C. Tarniţă, Computational verification of the Birch and Swinnerton-Dyer conjecture for individual elliptic curves, Math. Comp. 78 (2009), no. 268, 2397-2425, DOI 10.1090/S0025-5718-09-02253-4. MR 2521294 (2010g:11106)

[Gro91] B. H. Gross, Kolyvagin's work on modular elliptic curves, L-functions and arithmetic (Durham, 1989), London Math. Soc. Lecture Note Ser., vol. 153, Cambridge Univ. Press, Cambridge, 1991, pp. 235-256, DOI 10.1017/CBO9780511526053.009. MR 1110395 (93c:11039)

[GK92] B. H. Gross and S. S. Kudla, Heights and the central critical values of triple product L-functions, Compositio Math. 81 (1992), no. 2, 143-209. MR1145805 (93g:11047)

[GrZa] B. H. Gross and D. B. Zagier, Heegner points and derivatives of L-series, Invent. Math. 84 (1986), no. 2, 225-320, DOI 10.1007/BF01388809. MR833192 (87j:11057)

[H1] R. Hain, Lectures on the Hodge-de Rham theory of the fundamental group of $\mathbf{P}^{1}-$ $\{0,1, \infty\}$. http://math.arizona.edu/ swc/notes/files/05HainNotes.pdf.

[H2] R. M. Hain, The geometry of the mixed Hodge structure on the fundamental group, Algebraic geometry, Bowdoin, 1985 (Brunswick, Maine, 1985), Proc. Sympos. Pure Math., vol. 46, Amer. Math. Soc., Providence, RI, 1987, pp. 247-282. MR927984 (89g:14010)

[Ka] E. Kani, Endomorphisms of Jacobians of modular curves, Arch. Math. (Basel) 91 (2008), no. 3, 226-237, DOI 10.1007/s00013-008-2696-7. MR2439596 (2009j:11097)

[Ko] V. A. Kolyvagin, Finiteness of $E(\mathbf{Q})$ and $S H(E, \mathbf{Q})$ for a subclass of Weil curves (Russian), Izv. Akad. Nauk SSSR Ser. Mat. 52 (1988), no. 3, 522-540, 670-671; English transl., Math. USSR-Izv. 32 (1989), no. 3, 523-541. MR954295 (89m:11056)

[La] A. Lauder, Efficient computation of Rankin p-adic L-functions, in Computations with Modular Forms, Proceedings of a Summer School and Conference, Heidelberg, August/September 2011, Boeckle G. and Wiese G. (eds), Springer Verlag, to appear. 
[LoWe] D. Loeffler and J. Weinstein, On the computation of local components of a newform, Math. Comp. 81 (2012), no. 278, 1179-1200, DOI 10.1090/S0025-5718-2011-02530-5. MR:2869056 (2012k:11064)

[Mal] G. Malajovich, Condition number bounds for problems with integer coefficients, J. Complexity 16 (2000), no. 3, 529-551, DOI 10.1006/jcom.2000.0552. Complexity theory, real machines, and homotopy (Oxford, 1999). MR1787884 (2002g:65180)

[Man] Ju. I. Manin, Parabolic points and zeta functions of modular curves (Russian), Izv. Akad. Nauk SSSR Ser. Mat. 36 (1972), 19-66. MR0314846 (47 \#3396)

[MSD74] B. Mazur and P. Swinnerton-Dyer, Arithmetic of Weil curves, Invent. Math. 25 (1974), 1-61. MR0354674 (50 \#7152)

[O] A. P. Ogg, On the Weierstrass points of $X_{0}(N)$, Illinois J. Math. 22 (1978), no. 1, 31-35. MR0463178 (57 \#3136)

[Pr90] D. Prasad, Trilinear forms for representations of GL(2) and local $\epsilon$-factors, Compositio Math. 75 (1990), no. 1, 1-46. MR1059954 (91i:22023)

[R] H. Rademacher, The Fourier coefficients of the modular invariant $J(\tau)$, Amer. J. Math. 60 (1938), no. 2, 501-512, DOI 10.2307/2371313. MR1507331

[RS] K. A. Ribet, W. A. Stein, Lectures on modular forms and Hecke operators, available at http://wstein.org/books.

[Sil] J. H. Silverman, The Arithmetic of Elliptic Curves, 2nd ed., Graduate Texts in Mathematics, vol. 106, Springer, Dordrecht, 2009. MR2514094(2010i:11005)

$\left[\mathrm{S}^{+}\right.$09] W. A. Stein et al., Sage Mathematics Software (Version 4.7.1), The Sage Development Team, 2011, http://www.sagemath.org.

[Stn] W. Stein, Modular Forms, a Computational Approach, Graduate Studies in Mathematics, vol. 79, American Mathematical Society, Providence, RI, 2007. With an appendix by Paul E. Gunnells. MR2289048 (2008d:11037)

[Stv] G. Stevens, Arithmetic on Modular Curves, Progress in Mathematics, vol. 20, Birkhäuser Boston Inc., Boston, MA, 1982. MR670070 (87b:11050)

[Wa] M. Waldschmidt, Nombres Transcendants et Groupes Algébriques (French), Astérisque, vol. 69, Société Mathématique de France, Paris, 1979. With appendices by Daniel Bertrand and Jean-Pierre Serre; With an English summary. MR.570648 (82k:10041)

[Wat02] M. Watkins, Computing the modular degree of an elliptic curve, Experiment. Math. 11 (2002), no. 4, 487-502 (2003). MR1969641(2004c:11091)

[YZZ] X. Yuan, S. Zhang, W. Zhang, Triple product L-series and Gross-Schoen cycles I: split case, preprint.

[Zh] S. Zhang, Heights of Heegner points on Shimura curves, Ann. of Math. (2) 153 (2001), no. 1, 27-147, DOI 10.2307/2661372. MR1826411 (2002g:11081)

Department of Mathematics, McGill University, Montreal, Canada

E-mail address: darmon@math.mcgill.ca

Department of Mathematics, University of California at Berkeley, Berkeley, CalIFORNIA

E-mail address: mwdaub@math.berkeley.edu

University of California at Stanford, Stanford, California

E-mail address: saml@math.stanford.edu

Department of Mathematics, Universitat Politècnica de Catalunya, Barcelona, Spain

E-mail address: victor.rotger@upc.edu 